\title{
Experimental Gas-Phase Basicity Scale of Superbasic
}

\section{Phosphazenes}

Ivari Kaljurand, ${ }^{\dagger}$ Ilmar A. Koppel ${ }^{\dagger}$ Agnes Kütt, ${ }^{\dagger}$ Eva-Ingrid Rõõm,${ }^{\dagger}$ Toomas Rodima, ${ }^{\dagger}$ Ivar Koppel, ${ }^{\dagger}$ Masaaki Mishima, ${ }^{\ddagger}$ and Ivo Leito ${ }^{\dagger, *}$

University of Tartu, Department of Chemistry, Jakobi 2 str, 51014 Tartu, Estonia. Kyushu University, Institute for Materials Chemistry and Engineering, Hakozaki, Higashi-ku, Fukuoka 812-8581, Japan

\section{Supporting Information}

\section{Table of Contents}

Instrumentation, experimental setup and conditions of FT-ICR experiments.

... Page S2

Determination of $\mathrm{p} K_{\mathrm{ip}}(\mathrm{THF})$ and $\mathrm{p} K_{\alpha}(\mathrm{THF})$ and $\mathrm{p} K_{\mathrm{a}}(\mathrm{AN})$ values

Page S3

Table S2. Experimental and calculated gas-phase basicities, and experimental basicities in THF, AN and water.

... Page S6

Table S3. Results of calculations of gas-phase basicity (GB) in $\mathrm{kJ} / \mathrm{mol}$ of some strong bases at DFT B3LYP/6-311+G** level and experimental basicity values in various media.

... Page S7

Correlations of GBexp with solution basicities

... Page S11

Correlation $\mathrm{GB}_{\exp }$ with $\mathrm{p} K_{\mathrm{ip}}(\mathrm{THF})$ and $\mathrm{p} K_{\alpha}(\mathrm{THF})$

... Page S12

Correlation of $\mathrm{GB}_{\exp }$ with $\mathrm{p} K_{\mathrm{a}}(\mathrm{AN})$ and $\mathrm{p} K_{\mathrm{a}}\left(\mathrm{H}_{2} \mathrm{O}\right)$

... Page S16

Influence of the structure variation on the solvation of $\mathrm{P}_{1}$ (pyrr) series

.. Page S19

Footnotes

... Page S22

References

... Page S22

Optimized geometries obtained from the computations

... Page S25 


\section{Instrumentation, experimental setup and conditions of FT-ICR experiments.}

The FT-ICR spectrometric GB measurements were carried out at Kyushu University. Each equilibrium measurement is a measurement of relative basicity of bases $\mathrm{B}_{1}$ and $\mathrm{B}_{2}$ (that is "each arrow" in Table $\mathrm{S} 2$ ) according to the eqs $3-5$ of the manuscript. The partial pressures of the neutrals were measured using the Bayard-Alpert gauge and were corrected for the differences in ionization cross-sections (see ref 1 for details). Ratio of the intensities of the $\mathrm{M}+\mathrm{H}^{+}$ions in the mass spectrum was used as the estimate of the ratio of partial pressures of the ions. $\mathrm{M}+$ isotope intensity corrections for $\mathrm{M}+\mathrm{H}^{+}$ intensities were made when necessary. The eternal problem with introduction of compounds with low volatility is that the conventional leak-valve system is unsuitable for their introduction due to the long "path" from the inlet system (the socalled "oven") to the ICR cell. To solve this problem we used the so-called direct inlet probe. This is a stainless steel rod with diameter around $1 \mathrm{~cm}$. At its end there is a hole with $2 \mathrm{~mm}$ diameter to fix a capillary with the compound under study. The rod can be inserted into the ICR spectrometer in such a way that its tip is located at few centimeters from the cell (depending on the depth of introduction). The rod is inserted through a differentially pumped vacuum chamber to prevent degradation of the vacuum in the ICR cell. Even the compounds that have low vapor pressures can be vaporized this way to yield sufficient vapor pressure in the cell. The compounds $2,4,8,11,15,16,17,19,20,25-28,30$ were introduced by means of the direct inlet probe. ${ }^{1}$ Constant partial pressures of the compounds were obtained by cooling or heating the probe tip. Temperature was taken up slowly, and the temperature was kept constant after the compound's pressure was at appropriate level. Probe tip cooling was achieved by letting nitrogen gas generated from liquid nitrogen flow through the cooling gas canal of the probe. Direct inlet probe tip temperatures and net system pressure for introducing each of these compounds are given in Table S1. Depending on particular compound it took several hours to several days to enter the ICR cell. The rest of the bases and also 30 in some experiments were introduced from the conventional sample introduction system (the "oven") that was maintained at $150{ }^{\circ} \mathrm{C}$. The ICR cell temperature was kept $100 \pm 3{ }^{\circ} \mathrm{C}$. 
Table S1. Conditions for introduction of compounds of low volatility.

\begin{tabular}{llcc}
\hline No & Base & Probe tip temperature $\left({ }^{\circ} \mathrm{C}\right)$ & Pressure $\cdot 10^{7}$ (torr) \\
\hline 2 & $\mathrm{PhP}_{2}(\mathrm{dma})$ & $50-71$ & $4.5-11$ \\
4 & $2-\mathrm{Cl}_{-} \mathrm{C}_{6} \mathrm{H}_{4} \mathrm{P}_{2}(\mathrm{dma})$ & 61 & $5.1-7.1$ \\
8 & $4-\mathrm{Me}_{2} \mathrm{~N}-\mathrm{C}_{6} \mathrm{H}_{4} \mathrm{P}_{1}($ pyrr $)$ & $58-86$ & $2.3-6.5$ \\
11 & $4-\mathrm{MeO}-\mathrm{C}_{6} \mathrm{H}_{4} \mathrm{P}_{1}($ pyrr $)$ & $81-112$ & $6.9-7.7$ \\
19 & $4-\mathrm{Br}_{-} \mathrm{C}_{6} \mathrm{H}_{4} \mathrm{P}_{1}($ pyrr $)$ & $98-99$ & $4.0-6.9$ \\
25 & $2-\mathrm{NO}_{2}-5-\mathrm{Cl}_{-}-\mathrm{C}_{6} \mathrm{H}_{3} \mathrm{P}_{1}($ pyrr $)$ & 130 & $4.7-5.1$ \\
26 & $4-\mathrm{CF}_{3}-\mathrm{C}_{6} \mathrm{H}_{4} \mathrm{P}_{1}($ pyrr $)$ & $87-97$ & $5.7-8.7$ \\
27 & $2,6-\mathrm{Cl}_{2}-\mathrm{C}_{6} \mathrm{H}_{3} \mathrm{P}_{1}($ pyrr $)$ & $83-86$ & $3.3-5.6$ \\
28 & $2-\mathrm{NO}_{2}-4-\mathrm{Cl}_{-}-\mathrm{C}_{6} \mathrm{H}_{3} \mathrm{P}_{1}($ pyrr $)$ & $126-131$ & $4.4-5.8$ \\
30 & $\mathrm{PhP}_{1}\left(\mathrm{dma}_{2} \mathrm{Me}^{2}\right.$ & 45 & 5 \\
\hline
\end{tabular}

The equilibrium measurements were carried out at different partial pressures of the neutrals. Good agreement was obtained between the $\Delta \Delta G_{\mathrm{b}}$ values. At given partial pressures of the neutrals the equilibrium measurements were carried out as series of pulse sequences with different reaction times. Each sequence consisted of generating ions (by an electron impact pulse from a few to $10 \mathrm{~ms}$ ), giving them time to react (reaction time) and detecting the ions (see ref 2 for more details). From the ion intensity ratios at different reaction times (and at constant partial pressures of the neutrals) time plots were constructed. From the time plots it was found that between 1 and $20 \mathrm{~s}$ of reaction time was necessary to reach the equilibrium (depending on the reacting bases and their partial pressures). To ensure that the equilibrium has been reached reaction times significantly longer that what was required to reach the plateau on the time plot were used in all cases. All experiments were carried out at cell temperatures $373 \mathrm{~K}$.

\section{Determination of $\mathrm{p} K_{\mathrm{ip}}(\mathrm{THF})$ and $\mathrm{p} K_{\alpha}(\mathrm{THF})$ and $\mathrm{p} K_{\mathrm{a}}(\mathrm{AN})$ values}

The $\mathrm{p} K_{\mathrm{ip}}(\mathrm{THF})$ and $\mathrm{p} K_{\alpha}(\mathrm{THF})$ values of 9,12 and $\mathrm{p} K_{\mathrm{a}}\left(\mathrm{AN}^{1}\right)$ values of $4, \mathrm{PhP}_{1}(\mathrm{dma})_{2} \mathrm{tmg}$ and $\mathrm{PhP}_{1}(\mathrm{tmg})$ were

\footnotetext{
${ }^{1}$ AN denotes acetonitrile.
} 
determined as follows. The experimental $\Delta \mathrm{p} K_{\mathrm{ip}}$ determination spectrophotometric method in THF has been described in detail earlier ${ }^{3}$ and it was used without modifications. The measurements are relative, i.e. basicity difference (expressed either as $\Delta \mathrm{p} K_{\mathrm{ip}}$ or $\Delta \mathrm{p} K_{\alpha}$ ) of the two bases $\mathrm{B}_{1}$ and $\mathrm{B}_{2}$ is studied:

$$
\begin{array}{ccc}
K_{\mathrm{d}}^{\mathrm{HB}_{1}^{+} \mathrm{A}^{-}} & K & 1 / K_{\mathrm{d}}^{\mathrm{HB}_{2}^{+} \mathrm{A}^{-}} \\
\mathrm{B}_{2}+\mathrm{HB}_{1}^{+} \mathrm{A}^{-} \rightleftarrows \mathrm{B}_{2}+\mathrm{HB}_{1}^{+}+\mathrm{A}^{-} \rightleftarrows \mathrm{HB}_{2}^{+}+\mathrm{B}_{1}+\mathrm{A}^{-} \rightleftarrows \mathrm{HB}_{2}^{+} \mathrm{A}^{-}+\mathrm{B}_{1}
\end{array}
$$

The $K_{\mathrm{d}}-\mathrm{S}$ are the dissociation constants of the respective ion pairs. The directly measured quantity is the relative ion-pair basicity $-\Delta \mathrm{p} K_{\mathrm{ip}}-$ of bases $\mathrm{B}_{1}$ and $\mathrm{B}_{2}$. It is expressed as follows:

$$
\Delta \mathrm{p} K_{\mathrm{ip}}=\mathrm{p} K_{\mathrm{ip}}\left(\mathrm{HB}_{2}^{+} \mathrm{A}^{-}\right)-\mathrm{p} K_{\mathrm{ip}}\left(\mathrm{HB}_{1}^{+} \mathrm{A}^{-}\right)=\log \frac{K \cdot K_{\mathrm{d}}^{\mathrm{HB}_{1}^{+} \mathrm{A}^{-}}}{K_{\mathrm{d}}^{\mathrm{HB}_{2}^{+} \mathrm{A}^{-}}}=\log \frac{a\left(\mathrm{HB}_{2}^{+} \mathrm{A}^{-}\right) \cdot a\left(\mathrm{~B}_{1}\right)}{a\left(\mathrm{HB}_{1}^{+} \mathrm{A}^{-}\right) \cdot a\left(\mathrm{~B}_{2}\right)}
$$

The $K_{\mathrm{d}}$ values were estimated using the Fuoss equation as described in refs 3 and 17. Ionic radii are given in Table S5 in Supporting Information. Using the $K_{\mathrm{d}}$ values the $\mathrm{p} K_{\alpha}$ (an estimate of the $\mathrm{p} K_{\mathrm{a}}$ ) can be found as follows:

$$
\Delta \mathrm{p} K_{\alpha}=\mathrm{p} K_{\alpha}\left(\mathrm{HB}_{2}^{+}\right)-\mathrm{p} K_{\alpha}\left(\mathrm{HB}_{1}^{+}\right)=\Delta \mathrm{p} K_{\mathrm{ip}}-\log \frac{K_{\mathrm{d}}^{\mathrm{HB}_{1}^{+} \mathrm{A}^{-}}}{K_{\mathrm{d}}^{\mathrm{HB}_{2}^{+} \mathrm{A}^{-}}}
$$

The $\mathrm{p} K_{\mathrm{a}}$ determination method in $\mathrm{AN}$ is in principle similar to that in THF. The only difference is that the $\Delta \mathrm{p} K_{\mathrm{a}}$ is obtained directly from experimental data (for details see refs $6,3,7$ ).

$$
\mathrm{B}_{2}+\mathrm{HB}_{1}^{+} \rightleftarrows \mathrm{HB}_{2}^{+}+\mathrm{B}_{1}
$$

The relative basicity of the two bases $\mathrm{B}_{1}$ and $\mathrm{B}_{2}\left(\Delta \mathrm{p} K_{\mathrm{a}}\right)$ is defined as follows:

$$
\Delta \mathrm{p} K_{\mathrm{a}}=\mathrm{p} K_{\mathrm{a}}\left(\mathrm{HB}_{2}^{+}\right)-\mathrm{p} K_{\mathrm{a}}\left(\mathrm{HB}_{1}^{+}\right)=\log \frac{a\left(\mathrm{HB}_{2}^{+}\right) \cdot a\left(\mathrm{~B}_{1}\right)}{a\left(\mathrm{HB}_{1}^{+}\right) \cdot a\left(\mathrm{~B}_{2}\right)}
$$

The preparation of solutions in THF and AN, and titration experiments were carried out in a professional glovebox in argon atmosphere, where the contents of $\mathrm{O}_{2}$ and $\mathrm{H}_{2} \mathrm{O}$ were less than 1 ppm. Solutions of methanesulfonic acid (in THF) and triflic acid (in AN) were used as acidic and solution of $\operatorname{EtP}_{2}(\mathrm{dma})$ (in both solvents) was used as basic titrant. The measurements were carried out in an external cell compartment, situated 
in the glovebox. The cell compartment was connected to the UV-Vis spectrophotometer by means of two quartz fiber optic cables. For both solvents the new experimental results are given in Table 2 in the main text. 
Table S2. Experimental and calculated gas-phase basicities, and experimental basicities in THF, AN and water.

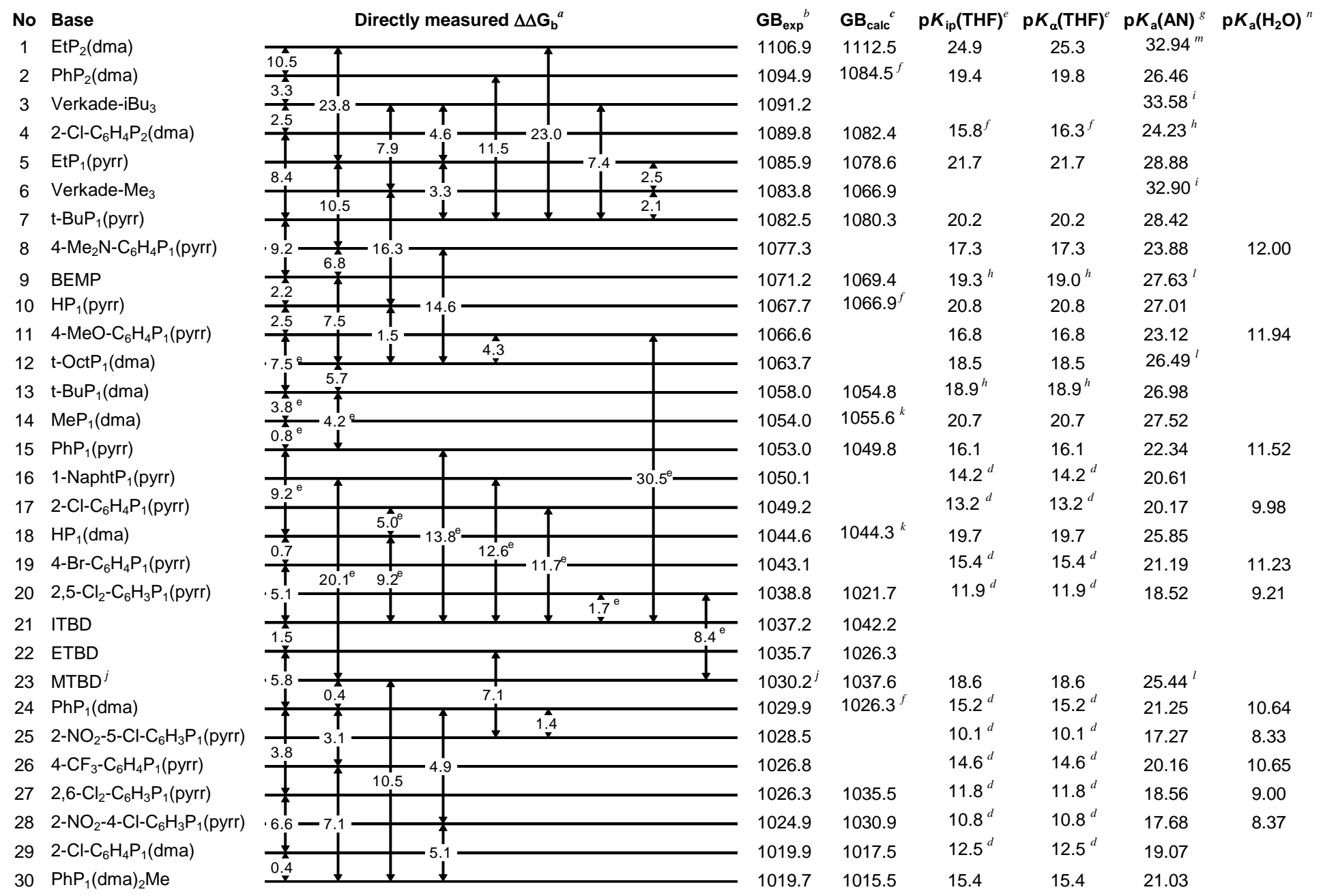

${ }^{a}$ Directly measured experimental $\Delta \Delta G_{\mathrm{b}}(\mathrm{kJ} / \mathrm{mol})$ values obtained from FT-ICR measurements. ${ }^{b}$ Estimated absolute GB (kJ/mol) values for bases. 1

$\mathrm{kcal} / \mathrm{mol}=4.184 \mathrm{~kJ} / \mathrm{mol} .{ }^{c}$ Results of basicity calculations at DFT B3LYP/6-311+G** level (kJ/mol), this work if not noted otherwise. ${ }^{d}$ Reference $3 .{ }^{e}$ Reference

4. ${ }^{f}$ Reference $5 .{ }^{g}$ Reference 6 if not noted otherwise. ${ }^{h}$ Present work. ${ }^{i}$ Reference $8 .{ }^{j}$ anchor of the scale, GB value taken from ref $9 .{ }^{k}$ Reference $10 .{ }^{l}$ Reference

11. ${ }^{m}$ Reference $12 .{ }^{n}$ Reference 13 
Table S3. Results of calculations of gas-phase basicity (GB) in kJ/mol of some strong bases at DFT B3LYP/6-311+G** level and experimental basicity

values in various media. ${ }^{a}$

\begin{tabular}{|c|c|c|c|c|c|c|c|c|c|c|c|c|c|c|}
\hline \multirow[b]{2}{*}{ No } & & \multicolumn{3}{|c|}{ Neutral } & \multicolumn{3}{|c|}{ Protonated Form } & \multirow[b]{2}{*}{$\mathrm{GB}_{\text {calc }}$} & \multirow[b]{2}{*}{$\mathrm{PA}(\mathrm{B})$} & \multirow[b]{2}{*}{$\mathrm{GB}_{\exp }$} & \multirow[b]{2}{*}{$\mathrm{p} K_{\mathrm{a}}(\mathrm{AN})^{c}$} & \multirow[b]{2}{*}{$\mathrm{p} K_{\mathrm{ip}}(\mathrm{THF})^{e}$} & \multirow[b]{2}{*}{$\mathrm{p} K_{\alpha}(\mathrm{THF})^{e}$} & \multirow[b]{2}{*}{$\mathrm{p} K_{\mathrm{a}}\left(\mathrm{H}_{2} \mathrm{O}\right)^{l}$} \\
\hline & & E & $\mathrm{H}$ & $\mathrm{G}$ & E & $\mathrm{H}$ & G & & & & & & & \\
\hline 18 & $\mathrm{HP}_{1}(\mathrm{dma})$ & -800.5860 & -800.3049 & -800.3633 & -801.0065 & -800.7110 & -800.7704 & $1044.3^{d}$ & $1072.4^{d}$ & 1044.6 & 25.85 & 19.7 & 19.7 & \\
\hline 14 & $\mathrm{MeP}_{1}(\mathrm{dma})$ & & & & & & & $1055.6^{d}$ & & 1054.0 & 27.52 & 20.7 & 20.7 & \\
\hline 13 & $\mathrm{t}-\mathrm{BuP}_{1}(\mathrm{dma})$ & -957.8684 & -957.4703 & -957.5408 & -958.2952 & -957.8820 & -957.9527 & 1054.8 & 1087.0 & 1058.0 & 26.98 & 18.9 & 18.9 & \\
\hline 9 & BEMP & -1074.6385 & -1074.1731 & -1074.2486 & -1075.0709 & -1074.5904 & -1074.6661 & 1069.4 & 1102.1 & 1071.2 & 27.63 & 19.3 & 19.0 & \\
\hline 10 & $\mathrm{HP}_{1}($ pyrr) & -1032.9040 & -1032.5106 & -1032.5781 & -1033.3353 & -1032.9270 & -1032.9947 & $1066.9^{d}$ & $1099.6^{d}$ & 1067.7 & 27.01 & 20.8 & 20.8 & \\
\hline 5 & $\operatorname{EtP}_{1}($ pyrr $)$ & -1111.5381 & -1111.0853 & -1111.1616 & -1111.9763 & -1111.5083 & -1111.5826 & 1078.6 & 1116.7 & 1085.9 & 28.88 & 21.7 & 21.7 & \\
\hline 7 & $\mathrm{t}-\mathrm{BuP}_{1}(\mathrm{pyrr})$ & -1190.1862 & -1189.6758 & -1189.7543 & -1190.6244 & -1190.0986 & -1190.1760 & 1080.3 & 1048.5 & 1082.5 & 28.42 & 20.2 & 20.2 & \\
\hline 30 & $\mathrm{PhP}_{1}(\mathrm{dma})_{2} \mathrm{Me}$ & -937.0049 & -936.6867 & -936.7518 & -937.4162 & -937.0837 & -937.1488 & 1015.5 & 1048.5 & 1019.7 & 21.03 & 15.4 & 15.4 & \\
\hline 24 & $\mathrm{PhP}_{1}(\mathrm{dma})$ & -1031.6909 & -1031.3243 & -1031.3947 & -1032.1055 & -1031.7246 & -1031.7958 & $1026.3^{d}$ & $1069.4^{d}$ & 1029.9 & 21.25 & 15.2 & 15.2 & 10.64 \\
\hline 29 & $2-\mathrm{Cl}-\mathrm{C}_{6} \mathrm{H}_{4} \mathrm{P}_{1}(\mathrm{dma})$ & -1491.3147 & -1490.9563 & -1491.0309 & -1491.7268 & -1491.3542 & -1491.4286 & 1017.5 & 1051.0 & 1019.9 & 19.07 & 12.5 & 12.5 & \\
\hline 15 & $\mathrm{PhP}_{1}$ (pyrr) & -1264.0093 & -1263.5301 & -1263.6094 & -1264.4344 & -1263.9406 & -1264.0195 & 1049.8 & 1057.3 & 1053.0 & 22.34 & 16.0 & 16.0 & 11.52 \\
\hline 27 & $\begin{array}{l}2,6-\mathrm{Cl}_{2^{-}} \\
\mathrm{C}_{6} \mathrm{H}_{3} \mathrm{P}_{1} \text { (pyrr) }\end{array}$ & -2183.2545 & -2182.7916 & -2182.8755 & -2183.6406 & -2183.1924 & -2183.2800 & 1035.5 & 1060.2 & 1026.3 & 18.56 & 11.8 & 11.8 & 9.00 \\
\hline 20 & $\begin{array}{l}2,5-\mathrm{Cl}_{2-} \\
\mathrm{C}_{6} \mathrm{H}_{3} \mathrm{P}_{1} \text { (pyrr) }\end{array}$ & -2183.2599 & -2182.7996 & -2182.8878 & -2183.6746 & -2183.1998 & 2183.2872 & 1021.7 & & 1038.8 & 18.52 & 11.9 & 11.9 & 9.21 \\
\hline 28 & $\begin{array}{l}2-\mathrm{NO}_{2}-4-\mathrm{Cl}- \\
\mathrm{C}_{6} \mathrm{H}_{3} \mathrm{P}_{1} \text { (pyrr) }\end{array}$ & -1928.1929 & -1927.7170 & -1927.8058 & -1928.6109 & -1928.1206 & -1928.2084 & 1030.9 & 1066.1 & 1024.9 & 17.68 & 10.8 & 10.8 & 8.37 \\
\hline & $\mathrm{PhP}_{1}(\mathrm{tmg})$ & -1714.1506 & -1713.4838 & -1713.6005 & -1714.6137 & -1713.9313 & -1714.0474 & 1146.4 & 1181.1 & & 31.4 & 23.7 & 24.3 & \\
\hline
\end{tabular}




\begin{tabular}{|c|c|c|c|c|c|c|c|c|c|c|c|c|}
\hline $\mathrm{EtP}_{2}(\mathrm{dma})$ & -1544.6159 & -1544.0909 & -1544.1839 & -1545.0661 & -1545.5251 & -1545.6178 & 1112.5 & 1146.4 & 1106.9 & 32.94 & 24.9 & 25.3 \\
\hline $\mathrm{PhP}_{2}(\mathrm{dma})$ & -1697.0862 & -1696.5346 & -1696.6319 & -1697.5243 & -1696.9575 & -1697.0551 & $1084.5^{d}$ & $1116.7^{d}$ & 1094.9 & 26.46 & 19.4 & 19.8 \\
\hline 2-Cl- $\mathrm{C}_{6} \mathrm{H}_{4} \mathrm{P}_{2}(\mathrm{dma})$ & -2156.7092 & -2156.1658 & -2156.2658 & -2157.1462 & -2156.5876 & -2156.6882 & 1082.4 & 1113.8 & 1089.8 & 24.23 & 15.8 & 16.3 \\
\hline $\begin{array}{l}\text { N, N', N" - } \\
\mathrm{Me}_{3} \text { Verkade's Base }\end{array}$ & -916.0987 & -915.7722 & -915.8297 & -916.5316 & -916.1899 & -916.2463 & 1066.9 & 1102.9 & 1083.8 & $32.90^{i}$ & & \\
\hline $\begin{array}{l}\text { N, N', N" - i- } \\
\text { Bu }_{3} \text { Verkade's Base }\end{array}$ & & & & & & & & & 1091.2 & $33.58^{i}$ & & \\
\hline TBD & -438.9452 & -438.7307 & -438.7736 & -439.3616 & -439.1322 & -439.1767 & 1031.4 & 1060.2 & $1022.1^{b}$ & 26.03 & 21.7 & 21.0 \\
\hline MTBD & -478.2595 & -478.0161 & -478.0626 & -478.6782 & -478.4195 & -478.4680 & 1037.6 & 1065.2 & $1030.2^{b}$ & 25.49 & 18.6 & 18.0 \\
\hline ETBD & -517.5850 & -517.3119 & -517.3617 & -518.0053 & -517.7169 & -517.7676 & 1026.3 & 1069.4 & 1035.7 & & & \\
\hline ITBD & -556.9052 & -556.6031 & -556.6559 & -557.3264 & -557.0089 & -557.0631 & 1042.2 & 1071.5 & 1037.2 & & & \\
\hline DBD & -422.8978 & -422.6722 & -422.7152 & -423.3116 & -423.0709 & -423.1144 & 1020.9 & 1053.1 & $1014.0^{b}$ & & & \\
\hline DBU & -462.2167 & -461.9611 & -462.0072 & -462.6267 & -462.3558 & -462.4020 & 1009.6 & 1042.2 & $1015.5^{b}$ & 24.34 & 18.1 & 16.9 \\
\hline DBN & -383.5734 & -383.9778 & -383.4188 & -383.9841 & -383.7736 & -383.4148 & 1012.5 & 1045.6 & $1005.9^{b}$ & 23.79 & & \\
\hline TTT & -570.5172 & -570.2722 & -570.3199 & -570.9511 & -570.6911 & -570.7399 & 1076.1 & 1106.2 & $1066.3^{f}$ & $26.22^{h}$ & & \\
\hline Guanidine & & & & & & & $964.8^{d}$ & $993.7^{d}$ & $949.4^{b}$ & & & \\
\hline TMG & & & & & & & $1007.1^{d}$ & $1038.5^{d}$ & $997.4^{b}$ & 23.3 & 17.0 & 15.5 \\
\hline PhTMG & -593.8014 & -593.5187 & -593.5763 & -594.2098 & -593.9122 & -593.9697 & 1006.3 & 1039.3 & $1006.0^{b}$ & 20.84 & 15.0 & 14.0 \\
\hline $\mathrm{NH}_{3}$ & & & & & & & $817.6^{m}$ & & $819.0^{b}$ & $16.46^{k}$ & & \\
\hline $\mathrm{MeNH}_{2}$ & & & & & & & $864.8^{m}$ & & $864.5^{b}$ & $18.37^{k}$ & & \\
\hline $\mathrm{H}_{2} \mathrm{NNH}_{2}$ & -111.9106 & -111.8531 & -111.8792 & -112.2505 & -112.1783 & -112.2056 & 830.1 & 859.8 & $822.4^{b}$ & $16.48^{k}$ & & \\
\hline $\mathrm{NCNH}_{2}$ & -148.8349 & -148.7964 & -148.8246 & -149.1533 & -149.1037 & -149.1327 & 782.0 & 813.0 & $774.9^{b}$ & $4.0^{k}$ & & \\
\hline$\left(\mathrm{CF}_{3}\right)_{3} \mathrm{CNH}_{2}$ & -1107.3140 & -1107.2222 & -1107.2754 & -1107.6194 & -1107.5132 & -1107.5669 & 738.1 & 770.3 & $752.9^{b}$ & & & \\
\hline
\end{tabular}




\begin{tabular}{|c|c|c|c|c|c|c|c|c|c|c|c|c|}
\hline $\mathrm{CF}_{3} \mathrm{CH}_{2} \mathrm{NH}_{2}$ & -433.0506 & -432.9737 & -432.0105 & -433.3804 & -433.2889 & -433.3259 & 801.2 & 833.5 & $812.9^{b}$ & $11.81^{k}$ & & \\
\hline $\mathrm{PhCH}_{2} \mathrm{NH}_{2}$ & -327.0026 & -326.8490 & -326.8889 & -327.3682 & -327.1997 & -327.2398 & 894.5 & 927.2 & $879.4^{b}$ & 16.91 & & \\
\hline 4-MeO-Aniline & -402.2407 & -402.0825 & -402.1249 & -402.5957 & -402.4226 & -402.4662 & 872.4 & 899.1 & $868.5^{b}$ & 11.86 & 8.3 & 6.5 \\
\hline 2-Me-Aniline & -327.0142 & -326.8618 & -326.9033 & -327.3656 & -327.1984 & -327.2416 & 866.5 & 889.9 & $859.1^{b}$ & 10.50 & 6.9 & 5.1 \\
\hline Aniline & & & & & & & $855.2^{m}$ & & $850.6^{b}$ & 10.62 & 7.0 & 5.2 \\
\hline 4- $\mathrm{CF}_{3}$-Aniline & -624.8398 & -624.7084 & -624.7548 & -625.1714 & -625.0252 & -625.0743 & 812.1 & & & 8.03 & & \\
\hline 4- $\mathrm{NO}_{2}$-Aniline & & & & & & & & & $834.2^{b}$ & 6.22 & & \\
\hline 1-Napht-NH ${ }_{2}$ & & & & & & & & & $875.1^{b}$ & 9.77 & & \\
\hline Pyrrolidine & -212.6460 & -212.5110 & -212.5465 & -213.0217 & -212.8714 & -212.9052 & 915.0 & 952.7 & $915.3^{b}$ & 19.56 & 15.3 & 13.5 \\
\hline $\mathrm{Me}_{3} \mathrm{~N}$ & & & & & & & $911.7^{m}$ & & $918.1^{b}$ & $17.61^{k}$ & & \\
\hline $\mathrm{Et}_{3} \mathrm{~N}$ & -292.4972 & -292.2822 & -292.3268 & -292.8866 & -292.6556 & -292.7004 & 954.0 & 987.0 & $951.0^{b}$ & 18.82 & 14.0 & 12.5 \\
\hline $\mathrm{NCNMe}_{2}$ & -227.4680 & -227.3704 & -227.4065 & -227.8084 & -227.6989 & -227.7357 & 837.6 & 868.6 & $821.4^{b}$ & & & \\
\hline $\mathrm{Ph}_{3} \mathrm{~N}$ & -749.8854 & -749.5924 & -749.6518 & -750.2444 & -749.9371 & -749.9958 & 875.7 & & $876.4^{b}$ & & & \\
\hline $\begin{array}{l}\mathrm{N}, \mathrm{N}-\mathrm{Me}_{2} \text {-Aniline } \\
\text { (DMA) }\end{array}$ & -366.3141 & -366.1318 & -366.1749 & -366.6837 & -366.4866 & -366.5296 & 904.6 & 937.6 & $909.2^{b}$ & 11.43 & 6.5 & 4.9 \\
\hline 4-MeO-DMA & & & & & & & & & $922.4^{b}$ & & & \\
\hline 4- $\mathrm{H}_{2} \mathrm{~N}-\mathrm{DMA}$ & -421.6861 & -421.4860 & -421.5321 & -422.0683 & -421.8543 & -421.8998 & $\begin{array}{c}938.5 \\
\left(\mathrm{Me}_{2} \mathrm{~N}\right)\end{array}$ & $\begin{array}{c}972.8 \\
\left(\mathrm{Me}_{2} \mathrm{~N}\right)\end{array}$ & $928.4^{b}$ & & & \\
\hline Proton Sponge & -653.9755 & -653.6677 & -653.7327 & -654.3793 & -654.0577 & -654.1149 & 1000.4 & 1030.1 & $995.8^{b}$ & 18.62 & 11.6 & 11.1 \\
\hline 4- $\mathrm{Me}_{2} \mathrm{~N}$-Pyridine & -382.3601 & -382.1894 & 382.2326 & 282.7573 & 382.5721 & 382.6152 & 977.8 & 1010.9 & $971.1^{b}$ & 17.95 & 13.0 & 11.2 \\
\hline 4- $\mathrm{NH}_{2}$-Pyridine & -303.6564 & -303.6207 & -303.7324 & -304.1214 & -303.9953 & -304.0304 & 955.2 & 989.5 & $947.8^{b}$ & 17.62 & & \\
\hline 3- $\mathrm{NH}_{2}$-Pyridine & -303.7275 & -303.6160 & -303.6518 & -304.1057 & -303.9800 & -304.0164 & 930.5 & 961.9 & $922.6^{b}$ & 14.17 & & \\
\hline 2- $\mathrm{NH}_{2}$-Pyridine & -303.7383 & -303.6266 & -303.6623 & -304.1132 & -303.9877 & -304.0238 & 934.3 & 951.4 & $915.3^{b}$ & 14.47 & & \\
\hline
\end{tabular}




\begin{tabular}{|c|c|c|c|c|c|c|c|c|c|c|c|}
\hline 2,6- $\mathrm{Me}_{2}$-Pyridine & -327.0134 & -326.8619 & -326.9038 & -327.3943 & -327.2285 & -327.2719 & 939.7 & 968.6 & $931.1^{b}$ & 14.13 & 8.8 \\
\hline 4-MeO-Pyridine & -362.9109 & -362.7823 & -362.8210 & -363.2913 & -363.1485 & -363.1875 & 935.5 & 967.8 & $929.8^{b}$ & 14.23 & 9.1 \\
\hline 2-MeO-Pyridine & -362.9209 & -362.7923 & -362.8313 & -363.2904 & -363.1477 & -363.1868 & 906.3 & 939.3 & $902.8^{b}$ & 9.93 & \\
\hline 2-Me-Pyridine & -287.6824 & -287.5598 & -287.5969 & -288.0570 & -287.9210 & -287.9563 & 917.1 & 954.8 & $917.3^{b}$ & 10.50 & 8.1 \\
\hline Pyridine & -248.3512 & -248.2576 & -248.2902 & -248.7191 & -248.6112 & -248.6433 & 900.4 & 934.3 & $898.1^{b}$ & 12.53 & 7.3 \\
\hline 3-Cl-Pyridine & -707.9726 & -707.8874 & -707.9235 & -708.3308 & -708.2316 & -708.2678 & 877.0 & 910.0 & $871.5^{b}$ & 9.55 & \\
\hline 2-Cl-Pyridine & -707.9766 & -707.8914 & -707.9274 & -708.3323 & -708.2332 & -708.2694 & 871.1 & 903.7 & $869.0^{b}$ & 6.79 & \\
\hline Imidazole & & & & & & & $912.1^{m}$ & & $909.2^{b}$ & $14.99^{k}$ & \\
\hline $\mathrm{Me}_{3} \mathrm{P}$ & & & & & & & $917.6^{d}$ & $949.3^{d}$ & $926.3^{b}$ & & \\
\hline $\mathrm{Et}_{3} \mathrm{P}$ & -579.1295 & -578.9198 & -578.9677 & -579.5108 & -579.2896 & -579.3381 & 945.6 & 977.0 & $952.0^{b}$ & & \\
\hline $\mathrm{MePh}_{2} \mathrm{P}$ & -844.7201 & -844.4872 & -844.5433 & -845.0988 & -844.8545 & -844.9097 & 935.1 & 970.7 & $939.7^{b}$ & 9.96 & \\
\hline $\mathrm{Ph}_{3} \mathrm{P}$ & -1036.4961 & -1036.2077 & -1036.2670 & -1036.8790 & -1036.5794 & -1036.6399 & 852.3 & 982.0 & $940.4^{b}$ & 7.61 & \\
\hline $\mathrm{Me}_{2} \mathrm{PhP}$ & -652.9421 & -652.7658 & -652.8115 & -653.3175 & 963.1298 & -653.1764 & 931.4 & 961.9 & $936.8^{b}$ & & \\
\hline
\end{tabular}

${ }^{a}$ Value from this work or from ref 4 if not noted otherwise. ${ }^{b}$ Reference $9 .{ }^{c}$ References $6,11,12$ if not noted otherwise. ${ }^{d}$ References 5 and $10 .{ }^{e}$ Reference $3,4,5 .{ }^{f}$ Reference

14. ${ }^{g}$ Reference $15 .{ }^{h}$ Reference $16 .{ }^{i}$ Reference $8 .{ }^{j}$ Reference $17 .{ }^{k}$ Reference $18,19 .{ }^{l}$ Reference $13 .{ }^{m}$ Reference 20 
Correlations of $\mathbf{G B}_{\text {exp }}$ with solution basicities. It is of great interest to observe the influence of different media on the acid-base properties of bases in general view and also what is different when one takes a look on some particular family (e.g. phosphazenes). The solvent influence on compounds basicity can be observed by correlating the basicity values in the gas-phase and solvent. The correlation equation has the following form:

$$
\mathrm{GB}(\mathrm{kJ} / \mathrm{mol})=a \mathrm{p} K(\mathrm{X})+b,
$$

where $\mathrm{p} K(\mathrm{X})$ denotes basicity value in a solvent $\mathrm{X}, a$ and $b$ are the slope and intercept, respectively. The correlation results for various sets of compounds from this work and Table S3 are presented in Table S4.

Table S4. The Correlation of Gas-Phase Basicities (GB) with respective solution basicity values (pK(X)) in Solution According to Eq 1 .

\begin{tabular}{|c|c|c|c|c|c|c|c|c|c|}
\hline $\mathrm{p} K(\mathrm{X})$ & & $a$ & $s(a)$ & $b$ & $s(b)$ & $S$ & $R^{2}$ & $n$ & $a / 5.705^{a}$ \\
\hline \multirow[t]{2}{*}{$\mathrm{p} K_{\mathrm{ip}}(\mathrm{THF})$} & All bases & 11.57 & 1.38 & 839.3 & 21.4 & 41.7 & 0.639 & 42 & 2.03 \\
\hline & All non-phosphazenes & 10.42 & 1.63 & 818.8 & 21.2 & 31.5 & 0.732 & 17 & 1.83 \\
\hline \multirow[t]{5}{*}{$\mathrm{p} K_{\alpha}(\mathrm{THF})$} & All bases & 10.63 & 0.92 & 860.1 & 13.4 & 34.8 & 0.748 & 47 & 1.86 \\
\hline & All phosphazenes & 5.17 & 0.85 & 968.7 & 14.6 & 16.0 & 0.615 & 25 & 0.91 \\
\hline & $\mathrm{P}_{1}($ pyrr) series & 5.137 & 0.741 & 972.5 & 11.7 & 10.0 & 0.800 & 14 & 0.90 \\
\hline & $4-\mathrm{X}-\mathrm{PhP}_{1}$ (pyrr) series & 18.28 & 0.63 & 760.2 & 10.1 & 1.4 & 0.997 & 5 & 3.20 \\
\hline & All non-phosphazenes & 8.46 & 1.37 & 861.4 & 14.9 & 31.0 & 0.657 & 22 & 1.48 \\
\hline \multirow[t]{7}{*}{$\mathrm{p} K_{\mathrm{a}}(\mathrm{AN})$} & All bases & 10.94 & 0.79 & 768.6 & 16.0 & 45.1 & 0.742 & 68 & 1.92 \\
\hline & All phosphazenes & 5.021 & 0.712 & 937.0 & 17.0 & 14.5 & 0.684 & 25 & 0.88 \\
\hline & $\mathrm{P}_{1}($ pyrr) series & 5.163 & 0.617 & 938.0 & 13.8 & 8.6 & 0.854 & 14 & 0.91 \\
\hline & $4-\mathrm{X}-\mathrm{PhP}_{1}$ (pyrr) series & 13.21 & 0.77 & 761.0 & 17.0 & 2.3 & 0.990 & 5 & 2.31 \\
\hline & All non-phosphazenes & 9.505 & 0.971 & 774.9 & 17.1 & 43.2 & 0.700 & 43 & 1.67 \\
\hline & Pyridines & 9.118 & 1.146 & 796.7 & 14.7 & 14.5 & 0.864 & 12 & 1.60 \\
\hline & PhTMG series & 13.08 & 3.43 & 737.7 & 70.7 & 3.7 & 0.879 & 4 & 2.29 \\
\hline
\end{tabular}


Correlation $\mathbf{G B}_{\exp }$ with $\mathbf{p} K_{\mathrm{ip}}(\mathrm{THF})$ and $\mathbf{p} K_{\alpha}(\mathrm{THF})$. The overall correlation between GB and $\mathrm{p} K_{\alpha}(\mathrm{THF})$ is rather poor. This can be explained by the large variation in the molecular structure and size of the molecules of compounds correlated. The same conclusion is true for the correlation of all phosphazene bases in which the correlation coefficient is even poorer. From this correlation of GB with $\mathrm{p} K_{\alpha}(\mathrm{THF})$ it appears that in global view, for the current data set the gas phase is equal or up to 2 times better differentiating medium of basicities compared to THF. On the other hand, for 4-substituted $\mathrm{PhP}_{1}$ (pyrr) series, where access of the solvent molecule into the protonation center is not affected by the substituents in the phenyl ring the same correlation is excellent and for this series the gas phase is 3.20 times better differentiating medium of basicities than THF. It can be concluded that for the $\mathrm{PhP}_{1}$ (pyrr) series moderate variation of the molecule size (or volume) $\mathrm{H}$ vs $\mathrm{MeO}$ or $\mathrm{Me}_{2} \mathrm{~N}$ in the periphery relative to the protonation center does not have substantial influence on the size, as it can be understood in terms of size-enhanced cation stabilization ability in the gas-phase. According to the 4-X$\mathrm{PhP}_{1}$ (pyrr) series correlation equation the $\mathrm{HP}_{1}$ (pyrr), $\mathrm{EtP}_{1}$ (pyrr) and $\mathrm{t}-\mathrm{BuP}_{1}$ (pyrr) are in $\mathrm{THF} 4.0,4.1$ and 2.7 $\mathrm{p} K_{\alpha}$ units stronger bases than expected. Thus, substitution of $\mathrm{H}$ (or Et) with $\mathrm{t}-\mathrm{Bu}$ or $\mathrm{Ph}$ lowers the cation solvation stabilization by solvent by 1.3 and $4 \mathrm{p} K_{\alpha}$ units, respectively. Next, when going from $\mathrm{PhP}_{1}$ (pyrr) to 1 $\mathrm{NaphtP}_{1}$ (pyrr) or introducing one or several $\mathrm{Cl}$ substituents into the ortho position of the phenyl ring the additional loss of stabilization is 1.7 and from 2.6 to $3.3 \mathrm{p} K_{\alpha}$ units, respectively. The largest loss of stabilization is due the introduction of $\mathrm{NO}_{2}$ into the ortho position of the phenyl ring: 3.7 to $4.6 \mathrm{p} K_{\alpha}$ units. It can be concluded, that the stabilization of protonated molecule by solvation is strongly influenced by immediate vicinity of the protonation center, more precisely its steric hindrance ability. 


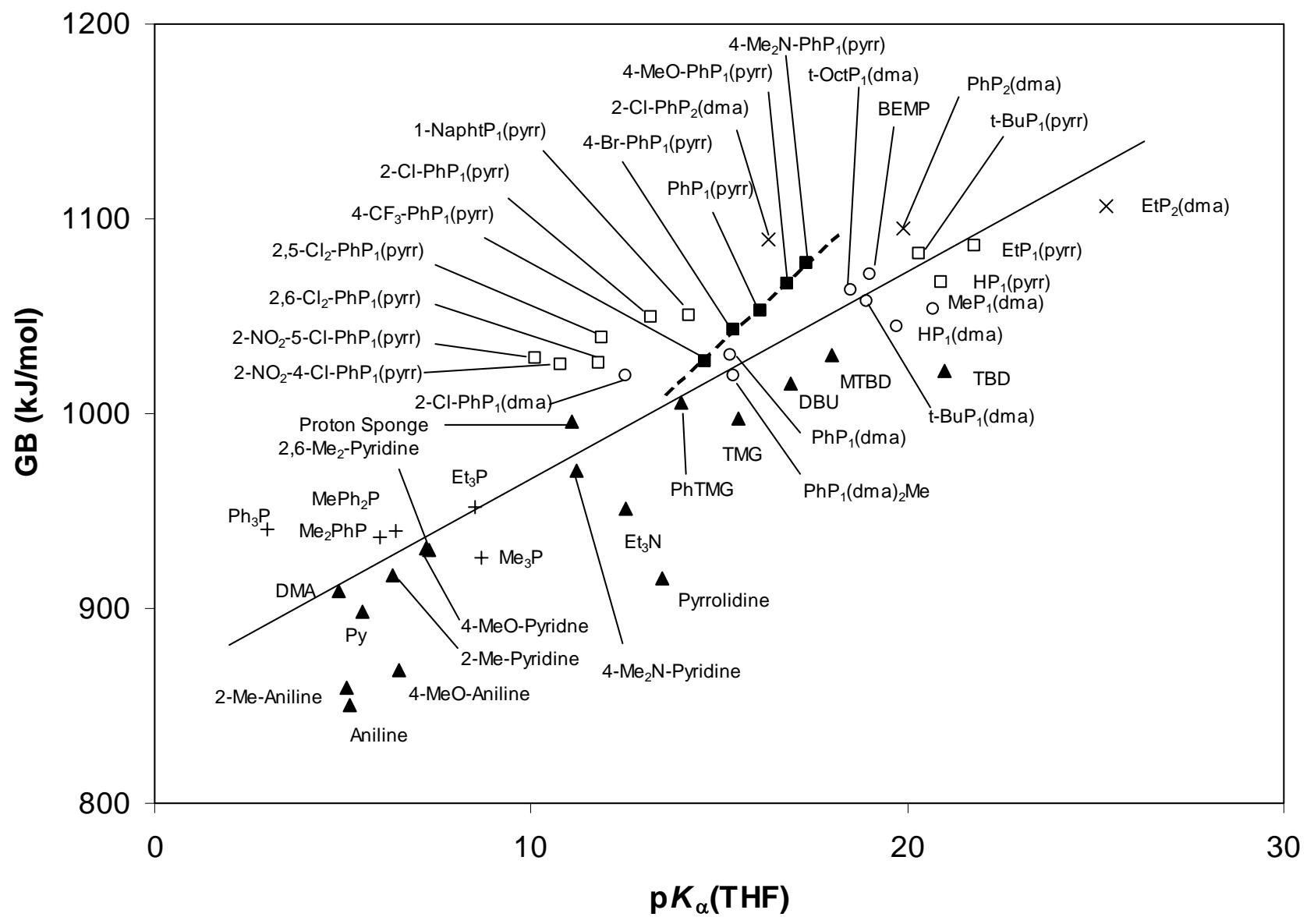

Figure S1. Comparison of the basicity values in THF ( $\mathrm{p} K_{\alpha}$ values) and in the gas phase (GB). The solid line corresponds to overall correlation (all available data included), $\mathrm{P}_{1}$ (pyrr) phosphazenes (14 compounds) from present work are denoted by $\square$, from which $4-\mathrm{X}-\mathrm{PhP}_{1}\left(\right.$ pyrr) phosphazenes by $\mathbf{m}, \mathrm{P}_{2}(\mathrm{dma})$ phosphazenes by $\times, \mathrm{P}_{1}(\mathrm{dma})$ phosphazenes by $\circ, \mathrm{N}$ bases $\boldsymbol{\Delta}$, and phosphines by + , respectively. The dashed line corresponds to the sub-series of $4-\mathrm{X}-\mathrm{PhP}_{1}$ (pyrr) phosphazenes.

Review of the most deviating points reveals that for many small molecules the correlation strongly overestimates the GB value. Examples are (in brackets: deviation of the experimental GB from the overall correlation line in $\mathrm{kJ} / \mathrm{mol})$ : pyrrolidine (-88.4), aniline (-64.8) and 4-methoxyaniline (-60.8). The large molecules on the other hand tend to have their GB values underestimated by the correlation. $2-\mathrm{NO}_{2}-5-\mathrm{Cl}-$ $\mathrm{C}_{6} \mathrm{H}_{3} \mathrm{P}_{1}$ (pyrr) (61.0), 2-Cl- $\mathrm{C}_{6} \mathrm{H}_{4} \mathrm{P}_{2}$ (dma) (56.3) and 2- $\mathrm{NO}_{2}-4-\mathrm{Cl}-\mathrm{C}_{6} \mathrm{H}_{3} \mathrm{P}_{1}$ (pyrr) (49.9) serve as examples. This leads to the idea of including the molecule/ion size in the correlation according to the following equation: 


$$
\mathrm{GB}(\mathrm{kJ} / \mathrm{mol})=a_{1} \mathrm{p} K(\mathrm{X})+a_{2} r+b
$$

, where $r$ is the mean ionic radius of the protonated base. Radii used in this correlation for these compounds are given in Table S5.

Table S5. Ionic radii of the protonated forms of the bases and methanesulfonate anion.

\begin{tabular}{|c|c|c|c|}
\hline ion & $\begin{array}{l}\text { ion-pair } \\
\text { radius, } \AA^{a}\end{array}$ & Ion & $\begin{array}{l}\text { ion-pair } \\
\text { radius, } \AA^{a}\end{array}$ \\
\hline $\mathrm{R}^{\prime} \mathrm{P}_{1}(\mathrm{pyrr}) \mathrm{H}^{+}, \mathrm{R}^{\prime} \mathrm{P}_{1}(\mathrm{dma}) \mathrm{H}^{+}$ & 4 & $\mathrm{TBDH}^{+}, \mathrm{MTBDH}^{+}, \mathrm{ETBDH}^{+}$, & 3 \\
\hline $\mathrm{R}^{\prime} \mathrm{P}_{1}(\mathrm{dma})_{2} \mathrm{MeH}^{+}, \mathrm{BEMPH}^{+}$ & & ITBDH $^{+}$, PhosphinesH $^{+}$ & \\
\hline $\mathrm{R}^{\prime} \mathrm{P}_{2}(\mathrm{dma}) \mathrm{H}^{+}$ & 4.8 & $\mathrm{PhP}_{1}(\mathrm{dma})_{2} \mathrm{tmg}$ & 4.5 \\
\hline $\mathrm{DBUH}^{+}$ & 2.5 & $\mathrm{PhTMGH}^{+}$ & 2.7 \\
\hline $\mathrm{TMGH}^{+} ; \mathrm{Et}_{3} \mathrm{NH}^{+}, \mathrm{N}, \mathrm{N}-\mathrm{Me}_{2^{-}}$ & 2.2 & X-AnilineH ${ }^{+}$; X-Pyridine $\mathrm{H}^{+}$, & 2 \\
\hline Aniline $^{+}, 2,6-\mathrm{X}_{2}$-Pyridine $\mathrm{H}^{+}$ & & PyrrolidineH $^{+}$ & \\
\hline $\mathrm{DMANH}^{+}$ & 3.2 & $\mathrm{CH}_{3} \mathrm{SO}_{3}^{-}$ & 2.5 \\
\hline
\end{tabular}

\footnotetext{
${ }^{a}$ Ionic radii from previous works (ref $3,5,17$ ) or this work. In cases when no data were available, the radii were estimated by PM3 calculations; R' = alkyl, aryl or $\mathrm{H} ; \mathrm{X}=$ substituent in phenyl ring.
}

This correlation leads to the following results: $\mathrm{GB}(\mathrm{kJ} / \mathrm{mol})=6.06 \mathrm{p} K_{\alpha}(\mathrm{THF})+41.7 r+783.0$, the standard deviations of the term $a_{1}, a_{2}$ and $b$ are $0.72,4.46$ and 11.5, respectively, indicating the significance of all the parameters in the correlation (see Table S6). Inclusion of the size parameter $r$ into the correlation equation of $\mathrm{p} K_{\alpha}(\mathrm{THF})$ and $\mathrm{GB}_{\text {exp }}$ improves the overall correlation in terms of $S$ and $R^{2}$, from 34.8 to 20.6 and from 0.748 to 0.914 respectively. The radius $r$ effectively takes into account the molecular volume/polarizability. It has been demonstrated $^{21}$ that molecular polarizability is an important parameter in determining reactivity in the gas phase and that it has lower influence on reactivity in condensed phase. 
Table S6. Correlation results for all compounds according to Eq 2 including the size parameter $r$.

\begin{tabular}{|c|c|c|c|c|c|c|c|c|c|}
\hline $\mathrm{p} K(\mathrm{X})$ & $a_{1}$ & $s\left(a_{1}\right)$ & $a_{2}$ & $s\left(a_{2}\right)$ & $b$ & $s(b)$ & $S$ & $R^{2}$ & $n$ \\
\hline $\begin{array}{l}\mathrm{p} K_{\alpha}(\mathrm{THF}) \text { all } \\
\text { compounds }\end{array}$ & 6.06 & 0.72 & 41.7 & 4.46 & 783.0 & 11.5 & 20.6 & 0.914 & 47 \\
\hline $\begin{array}{l}\mathrm{p} K_{\alpha}(\mathrm{THF}) \text { all } \\
\text { phosphazenes }\end{array}$ & 4.21 & 0.77 & 37.2 & 11.0 & 832.4 & 42.1 & 13.3 & 0.746 & 25 \\
\hline $\begin{array}{l}\mathrm{p} K_{\alpha}(\mathrm{THF}) \text { all } \\
\text { non- } \\
\text { phosphazenes }\end{array}$ & 7.29 & 1.16 & 41.8 & 12.2 & 770.5 & 29.7 & 25.7 & 0.777 & 22 \\
\hline $\begin{array}{l}\mathrm{p} K_{\mathrm{a}}(\mathrm{AN}) \text { all } \\
\text { compounds }\end{array}$ & 5.890 & 0.545 & 41.27 & 3.98 & 747.9 & 9.4 & 20.2 & 0.932 & 59 \\
\hline $\begin{array}{l}\mathrm{p} K_{\mathrm{a}}(\mathrm{AN}) \text { all } \\
\text { phosphazenes }\end{array}$ & 4.152 & 0.643 & 34.19 & 10.07 & 817.4 & 37.9 & 12.0 & 0.792 & 25 \\
\hline $\begin{array}{l}\mathrm{p} K_{\mathrm{a}}(\mathrm{AN}) \text { all } \\
\text { non- } \\
\text { phosphazenes }\end{array}$ & 6.432 & 0.822 & 40.71 & 10.03 & 740.5 & 18.3 & 23.9 & 0.888 & 34 \\
\hline
\end{tabular}

Similar trends, although not as extensive are observed also for the separate correlation sets of phosphazene and non-phosphazene bases (see Tables S4 and S6). Inclusion of the reciprocal $\left(r^{-1}\right)$ or reciprocal of square $\left(r^{-2}\right)$ of the size parameter $r$ into the correlation equation 7 instead of $r$ will not lead to improvement of the statistical parameters of the correlations. 


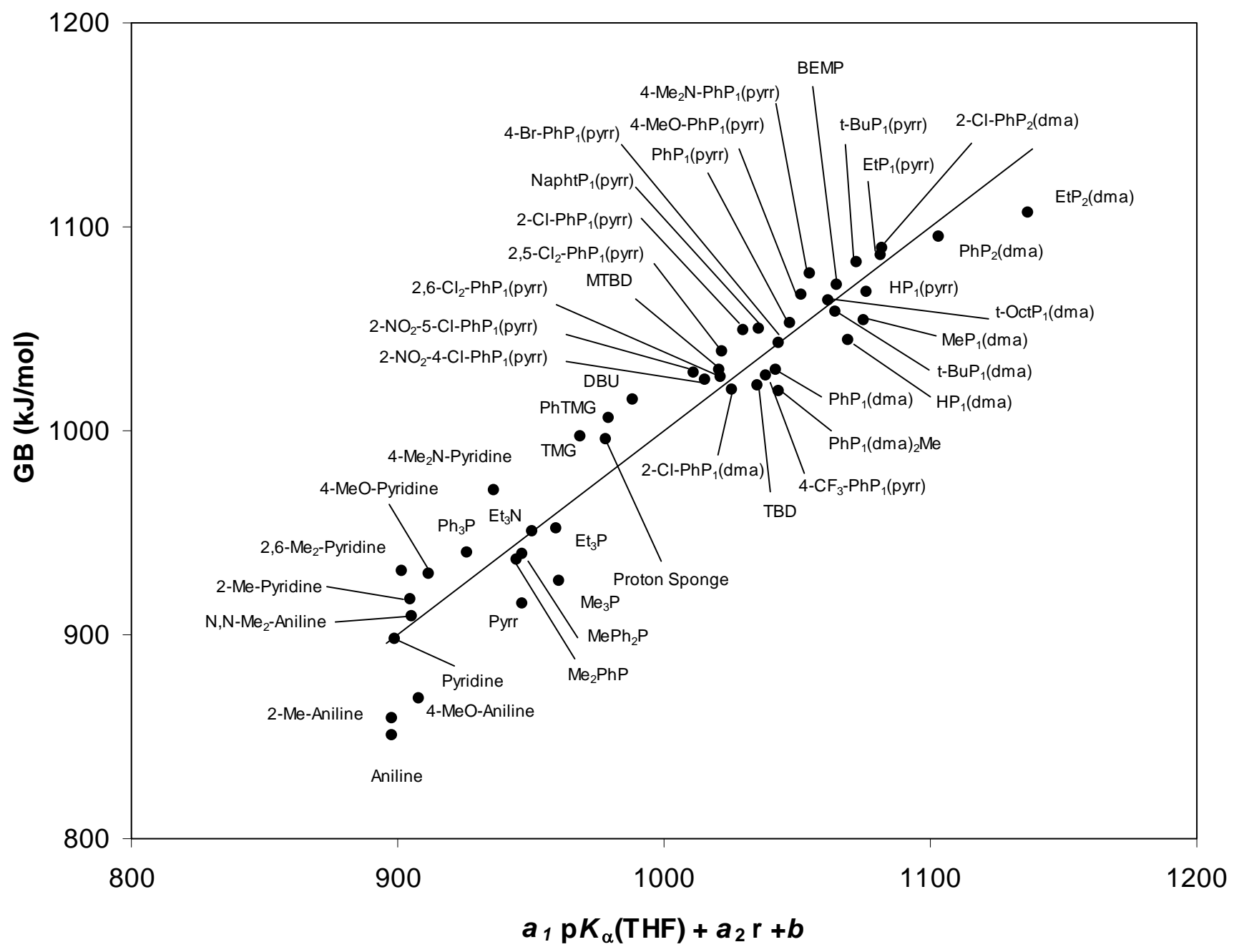

Figure S2. Correlation of the term $a_{1} \mathrm{p} K_{\alpha}(\mathrm{THF})+a_{2} r+b$ and the Gas-phase Basicity (GB). The solid line corresponds to overall correlation.

Correlation of $\mathrm{GB}_{\exp }$ with $\mathrm{p} K_{\mathrm{a}}(\mathrm{AN})$ and $\mathbf{p} K_{\mathrm{a}}\left(\mathrm{H}_{2} \mathrm{O}\right)$. The overall pattern of correlation of the GB values with $\mathrm{p} K_{\mathrm{a}}$ values in $\mathrm{AN}$ is similar to THF (see Figure $\mathrm{S} 3$ ). 


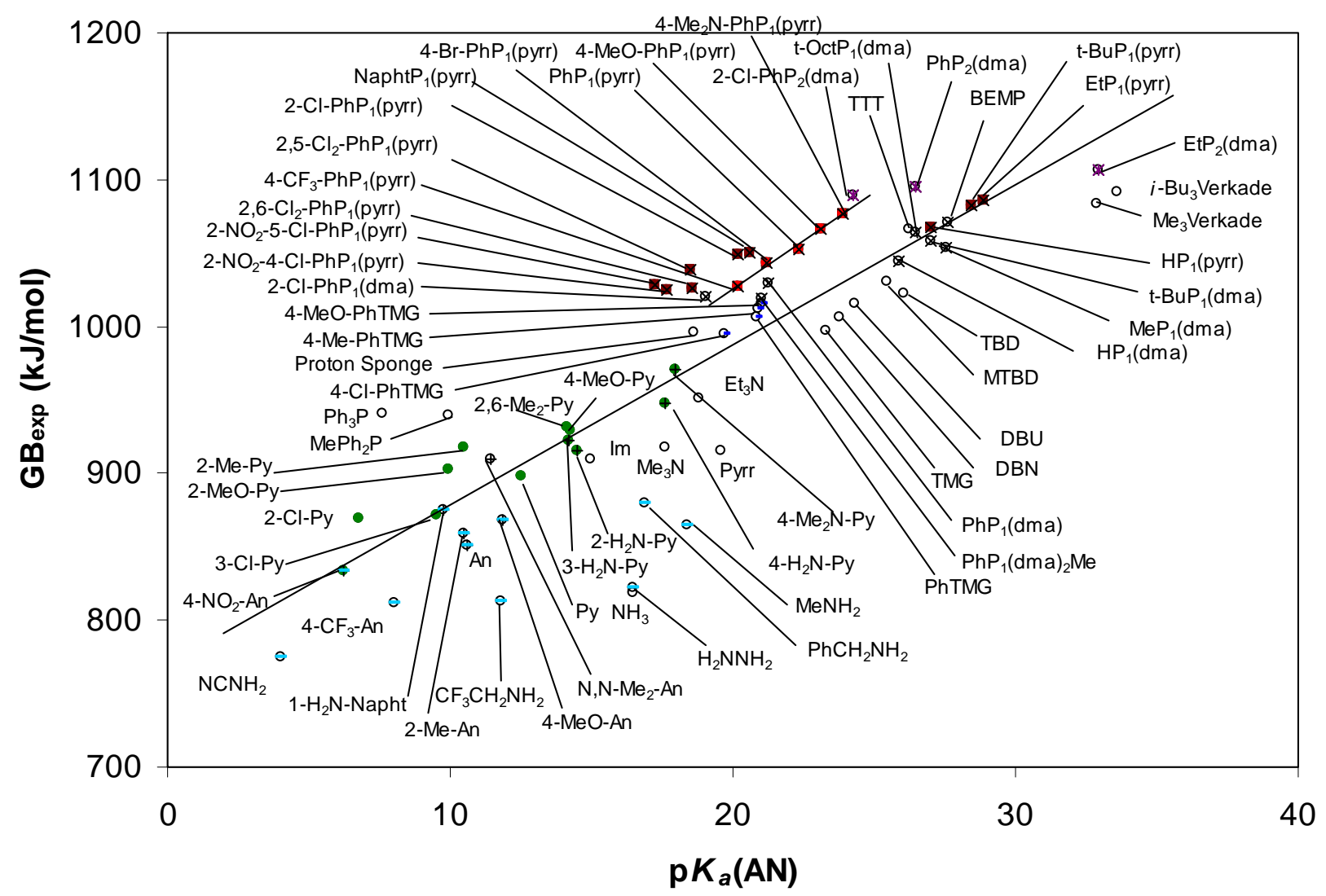

Figure S3. Correlation of the basicity values in $\mathrm{AN}$ ( $\mathrm{p} K_{a}$ values) and in the Gas Phase (GB). The solid line corresponds to overall correlation (all available data).

Molecular size plays an important role in this correlation as well. Correlation according to the eq 6 leads to the following results: $\mathrm{GB}_{\exp }=10.94 \mathrm{p} K_{\mathrm{a}}(\mathrm{AN})+768.6$, the standard deviations of the slope and intercept are 0.79 and 16.0, respectively, $R^{2}=0.742 ; n=68$ and $S=45.1$. Inclusion of molecule size parameter according to eq 7 gives $\mathrm{GB}_{\exp }=5.89 \mathrm{p} K_{\mathrm{a}}(\mathrm{AN})+41.3 r+747.9$, the standard deviations of the terms $a_{1}, a_{2}$ and $b$ are 0.55 , 3.98 and 9.4, respectively, $R^{2}=0.932 ; n=59$ and $S=20.2$. See Figure S4. Thus, including the molecule size in the correlation leads to a significant improvement in this solvent also. For the 4-substituted $\mathrm{PhP}_{1}$ (pyrr) series eq 6 is $\mathrm{GB}_{\exp }=13.21 \mathrm{p} K_{\mathrm{a}}(\mathrm{AN})+761.0$, the standard deviations of the slope and intercept are 0.77 and 17.0 , respectively, $R^{2}=0.990 ; n=5$ and $S=2.3$. Term $a / 5.705$ equals 2.31 , that is gas phase is 2.31 times better differentiator of basicities of $4-\mathrm{X}-\mathrm{PhP}_{1}$ (pyrr) series than $\mathrm{AN}$. 


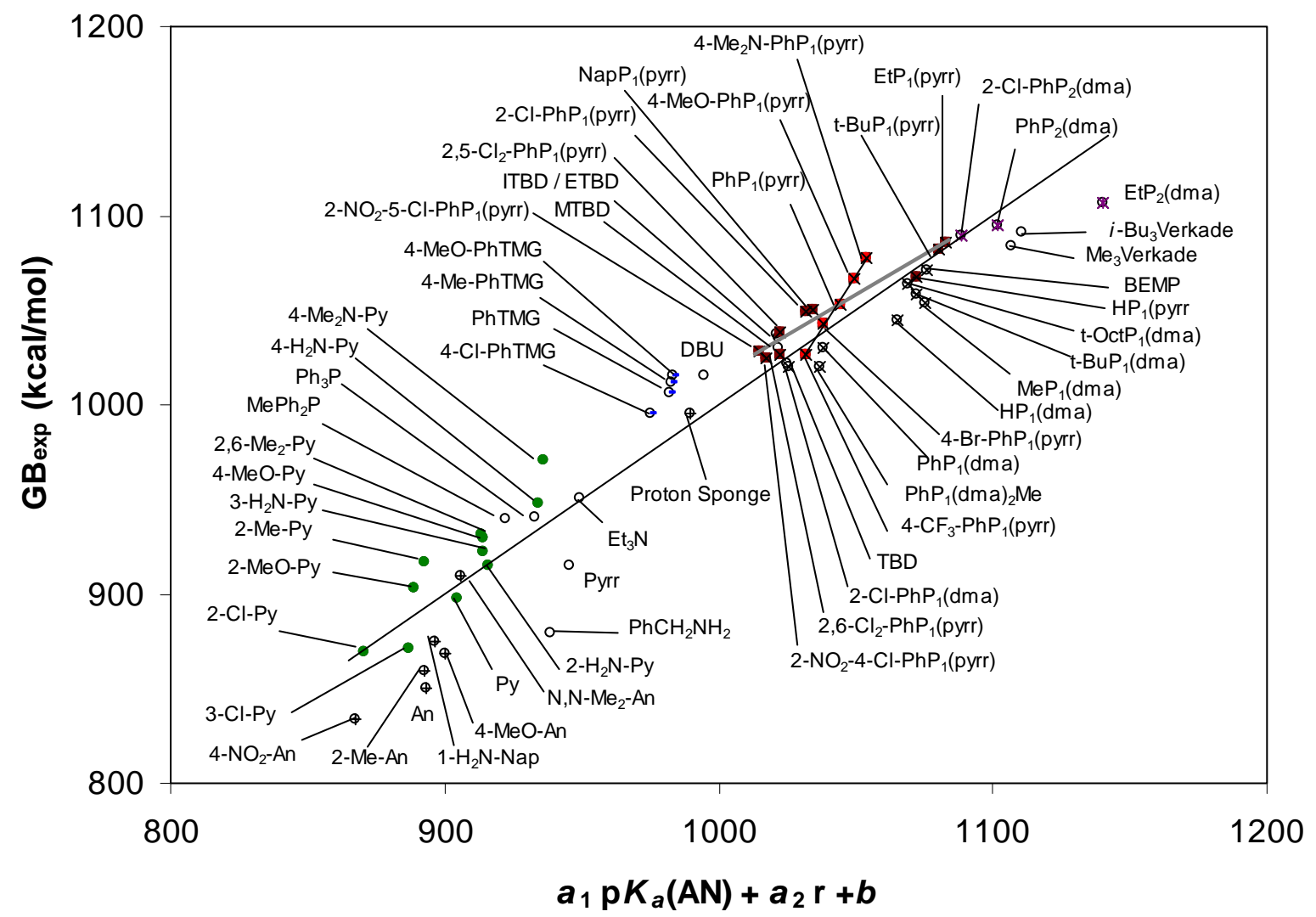

Figure S4. Correlation of the term $a_{1} \mathrm{p} K_{a}(\mathrm{AN})+a_{2} r+b$ and the Gas-phase Basicity (GB). The solid line corresponds to overall correlation (all available data).

Recently the $\mathrm{p} K_{\mathrm{a}}$ values for a set of $\mathrm{P}_{1}$ arylphosphazenes in water have been published. ${ }^{13}$ Their $\mathrm{p} K_{\mathrm{a}}$ values were found to be between 6 and 12 in water, meaning that in relative terms they are significantly weaker bases in water than in the gas phase. As an example: phenyltetramethylguanidine $\left(\mathrm{GB}_{\exp }=1006.0 \mathrm{~kJ} / \mathrm{mol}, \mathrm{p} K_{\mathrm{a}}\left(\mathrm{H}_{2} \mathrm{O}\right)=\right.$ 11.77) is a significantly weaker base in the gas phase than any of the studied $\mathrm{P}_{1}$ arylphosphazenes and at the same time beats most of them in the aqueous medium. Correlating the gas-phase data of this work with the $\mathrm{p} K_{\mathrm{a}}\left(\mathrm{H}_{2} \mathrm{O}\right)$ from ref 13 gives: $\mathrm{GB}(\mathrm{kJ} / \mathrm{mol})=10.32 \mathrm{p} K_{\mathrm{a}}\left(\mathrm{H}_{2} \mathrm{O}\right)+938.0$, the standard deviations of the slope and intercept are 2.58 and 26.6, respectively, $R^{2}=0.667 ; n=10$ and $S=11.1$. For the $4-\mathrm{X}-\mathrm{PhP}_{1}$ (pyrr) series the equation is as follows: $\mathrm{GB}(\mathrm{kJ} / \mathrm{mol})=34.96 \mathrm{p} K_{\mathrm{a}}\left(\mathrm{H}_{2} \mathrm{O}\right)+652.5$, the standard deviations of the slope and 
intercept are 3.72 and 42.7, respectively, $R^{2}=0.967 ; n=5$ and $S=4.1$. Term $a / 5.705$ equals 6.1 , meaning that the gas phase is 6.1 times better differentiator of basicities of the $4-\mathrm{X}-\mathrm{PhP}_{1}(\mathrm{pyrr})$ series than water. Although the correlation parameters of $4-\mathrm{X}-\mathrm{PhP}_{1}$ (pyrr) series for water are not as good as for $\mathrm{THF}$ and $\mathrm{AN}$ the certain trend of the influence ortho substituent hindrance can be observed also in comparison of this medium with the gas phase, see Figure S5.

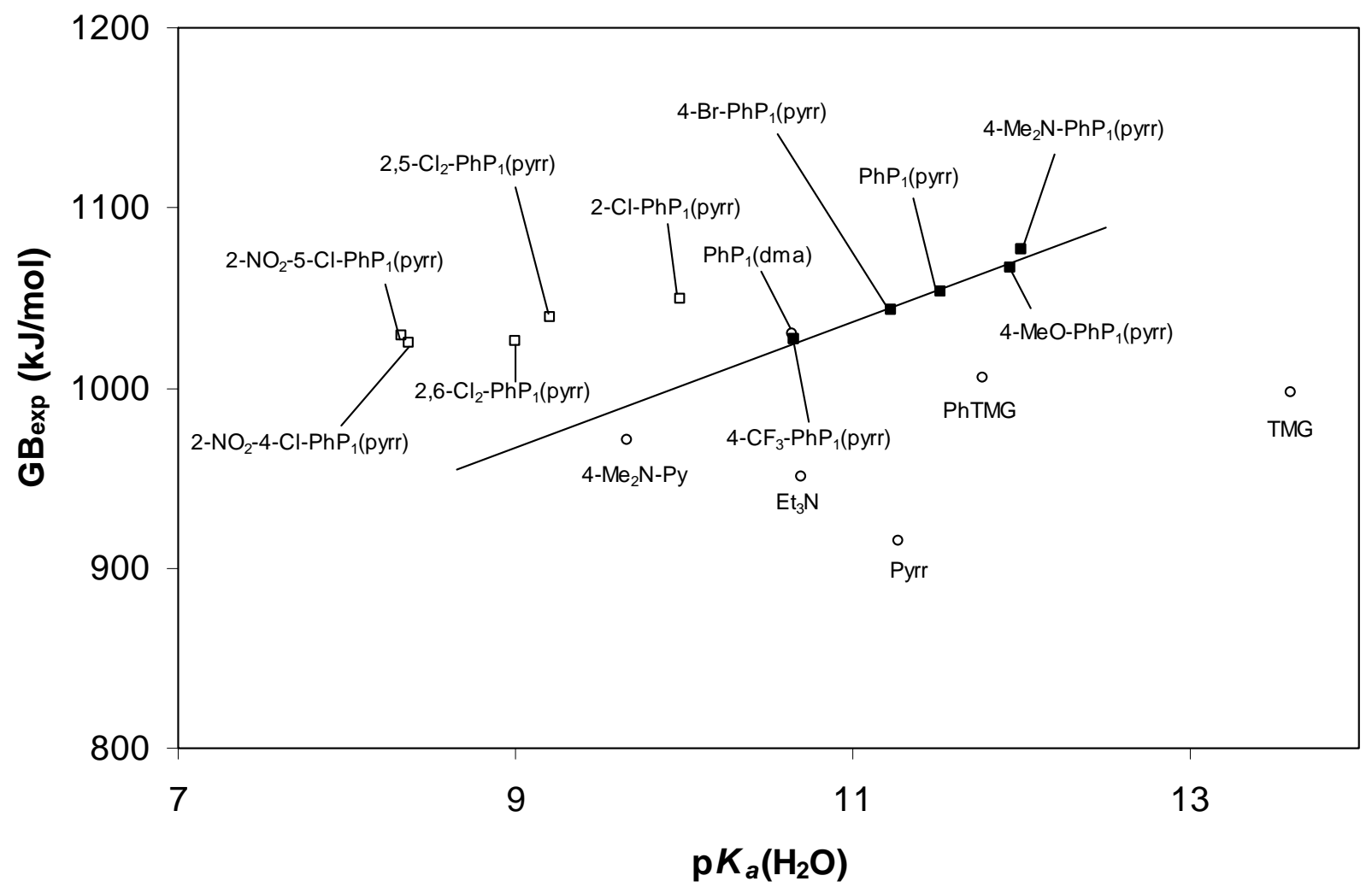

Figure S5. Correlation of the basicity values in water ( $\mathrm{p} K_{a}$ values) and in the Gas Phase of $P_{1}(p y r r)$ series and some other bases. The solid squares and solid line corresponds to $P_{1}(p y r r)$ compounds, open circles for some reference compounds. $\mathrm{p} K_{\mathrm{a}}\left(\mathrm{H}_{2} \mathrm{O}\right)$ data from ref 13.

Influence of the structure variation on the solvation of $\mathbf{P}_{\mathbf{1}}$ (pyrr) series. Previously we have studied ${ }^{6}$ influence of the structure variation on the $\mathrm{pK}$ values of the $\mathrm{P}_{1}$ (pyrr) phosphazene series in $\mathrm{AN}$ and THF. The overall correlation from the literature data ${ }^{6}$ given in Table $\mathrm{S} 7$ is: $\mathrm{p} K_{\alpha}(\mathrm{THF})=0.96 \mathrm{p} K_{\mathrm{a}}(\mathrm{AN})-5.8$, the standard deviations of the slope and intercept are 0.04 and 0.8 , respectively, $R^{2}=0.976 ; n=19$ and $S=0.67$. For 4 -X- 
$\mathrm{PhP}_{1}$ (pyrr) series the equation is as follows: $\mathrm{p} K_{\alpha}(\mathrm{THF})=0.74 \mathrm{p} K_{\mathrm{a}}(\mathrm{AN})-0.4$, the standard deviations of the slope and intercept are 0.02 and 0.5 , respectively, $R^{2}=0.996 ; n=6$ and $S=0.10$. This correlation supports the above-made observation, that when comparing one medium with another then para substituted $\mathrm{PhP}_{1}(\mathrm{pyrr})$ series forms a separate well-defined trend with much better correlation parameters, than the overall correlation. Using the $4-\mathrm{X}-\mathrm{PhP}_{1}$ (pyrr) series correlation equation parameters, we may calculate the estimated $\mathrm{p} K_{\alpha}(\mathrm{THF})$ values for all bases of the $\mathrm{P}_{1}$ (pyrr) series. The difference between experimental and estimated $\mathrm{p} K_{\alpha}(\mathrm{THF})$ values, which is given in the fourth column of Table S7, can be treated as a measure of relative (with respect to the 4-X$\mathrm{PhP}_{1}$ (pyrr) series) solvation change on transfer from AN to THF. Now, taking a closer look at this difference, we may conclude that the protonated forms of $4-\mathrm{X}-\mathrm{PhP}_{1}$ (pyrr) phosphazenes are in THF compared to AN by 1.2 $\mathrm{p} K_{\alpha}$ units less stabilized by solvent, than the protonated $\mathrm{HP}_{1}($ pyrr). Large ortho fragments inhibit stabilization by solvation of the protonated molecule even more. For example, compared to $4-\mathrm{X}-\mathrm{PhP}_{1}$ (pyrr) phosphazenes the loss is for 1-Naphthyl $0.7 \mathrm{p} K_{\alpha}$ units, for $\mathrm{Cl}$ in ortho position of phenyl ring from 1.3 to $2.5 \mathrm{p} K_{\alpha}$ units, for $\mathrm{NO}_{2}$ in same position from 1.9 to $2.6 \mathrm{p} K_{\alpha}$ units.

Table S7. The $\mathrm{p} K_{\mathrm{a}}(\mathrm{AN})$ and $\mathrm{p} K_{\alpha}(\mathrm{THF})$ Data from Ref 6.

\begin{tabular}{lccc}
\hline Compound & $\mathrm{p} K_{\mathrm{a}}(\mathrm{AN})$ & $\mathrm{p} K_{\alpha}(\mathrm{THF})$ & difference $^{a}$ \\
\hline $\mathrm{HP}_{1}($ pyrr $)$ & 27.01 & 20.8 & -1.18 \\
$\mathrm{EtP}_{1}$ (pyrr) & 28.88 & 21.7 & -0.70 \\
$t-\mathrm{ButP}_{1}$ (pyrr) & 28.42 & 20.2 & 0.46 \\
$4-\mathrm{Me}_{2} \mathrm{~N}_{-} \mathrm{C}_{6} \mathrm{H}_{4} \mathrm{P}_{1}$ (pyrr) & 23.88 & 17.3 & 0.00 \\
$4-\mathrm{MeO}-\mathrm{C}_{6} \mathrm{H}_{4} \mathrm{P}_{1}$ (pyrr) & 23.12 & 16.8 & -0.06 \\
$\mathrm{PhP}_{1}($ pyrr $)$ & 22.34 & 16.0 & 0.16 \\
$4-\mathrm{Br}_{-} \mathrm{C}_{6} \mathrm{H}_{4} \mathrm{P}_{1}$ (pyrr) & 21.19 & 15.4 & -0.09 \\
$4-\mathrm{CF}_{3}-\mathrm{C}_{6} \mathrm{H}_{4} \mathrm{P}_{1}$ (pyrr) & 20.16 & 14.6 & -0.05 \\
4- $\mathrm{NO}_{2}-\mathrm{C}_{6} \mathrm{H}_{4} \mathrm{P}_{1}$ (pyrr) & 18.51 & 13.3 & 0.05
\end{tabular}




\begin{tabular}{|c|c|c|}
\hline 1-NaphtP 1 (pyrr) & 20.61 & 14.2 \\
\hline $2-\mathrm{Cl}-\mathrm{C}_{6} \mathrm{H}_{4} \mathrm{P}_{1}$ (pyrr) & 20.17 & 13.2 \\
\hline $2,5-\mathrm{Cl}_{2}-\mathrm{C}_{6} \mathrm{H}_{3} \mathrm{P}_{1}$ (pyrr) & 18.52 & 11.9 \\
\hline $2,6-\mathrm{Cl}_{2}-\mathrm{C}_{6} \mathrm{H}_{3} \mathrm{P}_{1}$ (pyrr) & 18.56 & 11.8 \\
\hline $2,6-\mathrm{Cl}_{2}-4-\mathrm{NO}_{2}-\mathrm{C}_{6} \mathrm{H}_{2} \mathrm{P}_{1}$ (pyrr) & 14.43 & 7.8 \\
\hline $2-\mathrm{NO}_{2}-4-\mathrm{Cl}-\mathrm{C}_{6} \mathrm{H}_{3} \mathrm{P}_{1}$ (pyrr) & 17.68 & 10.8 \\
\hline $2-\mathrm{NO}_{2}-5-\mathrm{Cl}-\mathrm{C}_{6} \mathrm{H}_{3} \mathrm{P}_{1}$ (pyrr) & 17.27 & 10.1 \\
\hline $2-\mathrm{NO}_{2}-4-\mathrm{CF}_{3}-\mathrm{C}_{6} \mathrm{H}_{3} \mathrm{P}_{1}$ (pyrr) & 16.54 & 9.5 \\
\hline $2,4-\mathrm{NO}_{2}-\mathrm{C}_{6} \mathrm{H}_{3} \mathrm{P}_{1}$ (pyrr) & 14.88 & 8.0 \\
\hline $2,6-\mathrm{NO}_{2}-\mathrm{C}_{6} \mathrm{H}_{3} \mathrm{P}_{1}$ (pyrr) & 14.12 & 7.5 \\
\hline
\end{tabular}

${ }^{a}$ Difference $=\mathrm{p} K_{\mathrm{a}}(\mathrm{AN})$ times slope from $4-\mathrm{X}-\mathrm{Ph}(\mathrm{pyrr})$ series plus intercept from 4-X-Ph(pyrr) series minus $\mathrm{p} K_{\alpha}(\mathrm{THF})$, this quantity measures the difference between experimental and estimated $\mathrm{p} K_{\alpha}(\mathrm{THF})$ values.

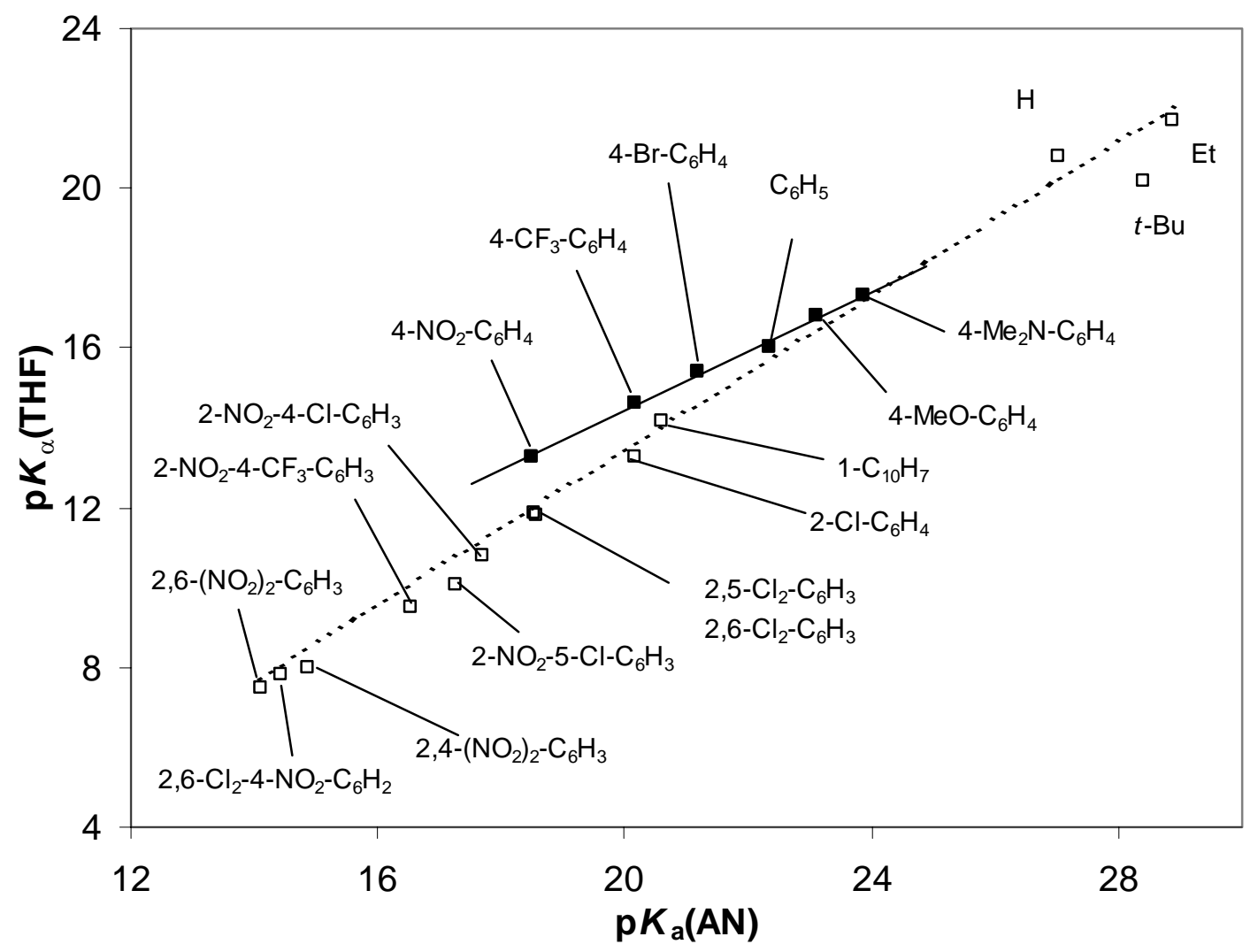

Figure S6. Correlation of the $\mathrm{p} K_{a}(\mathrm{AN})$ and $\mathrm{p} K_{\alpha}(\mathrm{THF})$ values of $\mathrm{P}_{1}(\mathrm{pyrr})$ series. The solid squares and solid line corresponds to 4-X-PhP 1 (pyrr) compounds, open squares and dotted line to the overall correlation. Data from ref 6. 


\section{Footnotes}

$\dagger$ University of Tartu

${ }^{\ddagger}$ Kyushu University.

*Corresponding author. E-mail: ivo.leito@ut.ee, Phone: +372 7375 259. Fax: +372 7375264.

\section{References}

1. Koppel, I. A.; Koppel, J.; Leito, I.; Koppel, I.; Mishima, M.; Yagupolskii, L. M. J. Chem. Soc., Perkin Trans. II, 2001, 229-232 and references therein.

2. Mishima, M.; Mustanir; Fujio, M.; Tsuno, Y. Bull. Chem. Soc. Jpn. 1996, 69, 2009-2018.

3. Rodima, T.; Kaljurand, I.; Pihl, A.; Mäemets, V.; Leito, I.; Koppel, I. J. Org. Chem. 2002, 67, 1873-1881.

4. Kaljurand, I.; Rodima, T.; Pihl, A.; Mäemets, V.; Leito, I.; Koppel, I.; Mishima, M. J. Org. Chem. 2003, 68, 9988-9993.

5. Kolomeitsev, A. A.; Koppel, I. A.; Rodima, T.; Barten, J.; Lork, E.; Röschenthaler, G.-V.; Kaljurand, I.; Kütt, A.; Koppel, I.; Mäemets, V.; Leito. I. J. Am. Chem. Soc. 2005, 50, 127, 17656 - 17666.

6. Kaljurand, I; Kütt, A.; Sooväli, L.; Rodima, T.; Mäemets, V.; Leito, I.; Koppel, I. A. J. Org. Chem. 2005, 70, 1019-1028.

7. Leito, I.; Kaljurand, I.; Koppel, I. A.; Yagupolskii, L. M.; Vlasov, V. M. J. Org. Chem., 1998, 63, 78687874. 
8. Kisanga, P. B.; Verkade, J. G.; Schwesinger, R. J. Org. Chem. 2000, 65, 5431-5432

9. Hunter, E. P. L.; Lias, S. G. J. Phys. Chem. Ref. Data. 1998, 27, 3, 413-656.

10. Koppel, I. A.; Schwesinger, R; Breuer, T.; Burk, P.; Herodes, K.; Koppel, I.; Leito, I.; Mishima, M. J. Phys. Chem. A 2001, 105, 9575-9586

11. Schwesinger, R., Willaredt, J., Schlemper, H., Keller, M., Schmitt,D., Fritz, H. Chem. Ber. 1994, 127, $2435-2454$.

12. Schwesinger, R.; Schlemper, H.; Hasenfratz, C.; Willaredt, J.; Dambacher, T.; Breuer, T.; Ottaway, C.; Fletschinger, M.; Boele, J.; Fritz, H.; Putzas, D.; Rotter, H. W.; Bordwell, F. G.; Satish, A. V.; Ji G.-Z.; Peters, E.-M.; Peters, K.; von Schnering, H. G.; Walz, L. Liebigs Ann. 1996, 1055-1081 and references therein.

13. Sooväli, L.; Rodima, T.; Kaljurand, I.; Kütt, A.; Koppel I. A. and Leito. I. Org. and Bioorg. Chem., 2006, 4, 2100-2105.

14. Raczyńska, E. D.; Decouzon, M.; Gal, J.-F.; Maria, P.-C.; Gelbard, G.; Vielfaure-Joly, F. J. Phys. Org. Chem. 2001, 14, 25-34.

15. Schwesinger, R.; Missfeldt, M.; Peters, K.; von Schnering, H. G. Angew. Chem., Int. Ed. Engl. 1987, 26, 1165-1167.

16. Schwesinger, R. Angew. Chem., Int. Ed. Engl. 1987, 26, 1164-1165.

17. Abdur-Rashid, K.; Fong, T. P.; Greaves, B.; Gusev, D. G.; Hinman, J. G.; Landau, S. E.; Lough, A. J.; Morris, R. H. J. Am. Chem. Soc. 2000, 122, 9155-9171.

18. Tables of Rate and Equilibrium Constants of Heterolytic Organic Reactions; Palm, V. Ed.; VINITI: Moscow-Tartu, 1975-1985.

19. Peips, M.; Koppel, J.; Pihl, V. O.; Koppel, I. A. Org. React. 1976, 13, 203-219.

20. Burk, P.; Koppel, I. A.; Koppel, I.; Leito, I. Travnikova, O. Chem. Phys. Lett. 2000, 323, 482-489.

21. Taft, R. W.; Topsom, R. D. Prog. Phys. Org. Chem. 1987, 16, 1. 
22. Raczyńska, E. D.; Decouzon, M.; Gal, J.-F.; Maria, P.-C.; Woźniak, K.; Kurg, R.; Carins, S. N. Trends in Organic Chemistry 1998, 7, 95-103.

23. Hansch, C.; Leo, A.; Taft, R. W. Chem. Rev. 1991, 91, 165-195. 
Optimized Geometries Obtained from the Computations

\section{$\mathrm{HP}_{1}$ (dma)}

dma3.log.acs

Brutoformula: C6H19N4P1

0,1

$\mathrm{H},-0.9920498368,1.2167456835,-2.3729831514$ $\mathrm{P},-0.3430369858,0.3635632616,-0.444536955$

$\mathrm{N}, 1.3540492855,0.4403979808,-0.5879537804$

N,-1.1149284863,1.3593310943,-1.3776626846

$\mathrm{N},-0.5521840117,-1.3341475808,-0.5486309191$

N,-0.8728092321,0.689104712,1.1240904205

C, $-1.6402457447,1.8969323256,1.4233318486$

C, $-0.1984507158,0.1066111787,2.2763422723$

C, $-1.8601004089,-1.8662085219,-0.1509014606$

C, $-0.0621102411,-1.9888526562,-1.7661817528$

C, $1.9661834788,1.7111771092,-0.9726421085$

C, $2.2916091723,-0.4733938605,0.05865318$

H,-2.4173980435,1.660249094,2.1597989719

$\mathrm{H},-2.1046172473,2.2630312323,0.5109621252$

$\mathrm{H},-1.0035534975,2.6900016766,1.840941235$

H, $-0.9228104928,-0.0393085408,3.085282227$

$\mathrm{H}, 0.6079244438,0.7484629375,2.6608657876$

$\mathrm{H}, 0.2202403405,-0.868354871,2.027453631$

$\mathrm{H},-0.7598952716,-1.8689501443,-2.6085108872$

$\mathrm{H}, 0.0554537677,-3.0604437121,-1.5766590657$

$\mathrm{H}, 0.9045424819,-1.5825922163,-2.0595966644$

$\mathrm{H},-2.6241440744,-1.7102316662,-0.9256748683$

$\mathrm{H},-2.1986242065,-1.3884450939,0.76642757$

$\mathrm{H},-1.765284951,-2.9419385228,0.0269642541$

H,3.1223826836,-0.6935471996,-0.6231589836

H, 1.8000136962,-1.4108369893,0.3115775263

H,2.7200097097,-0.0416882,0.9744488693

$\mathrm{H}, 2.7731815545,1.5328121022,-1.6933055718$

$\mathrm{H}, 2.3966392452,2.2337627786,-0.1060237237$

H, 1.2200135897,2.3567566088,-1.4327173414

$\mathrm{HF}=-800.5856456 \mathrm{NImag}=0$

\section{t-BuP $\mathbf{P}_{\mathbf{1}}(\mathbf{d m a})$}

tbup1.log.acs

\section{Brutoformula: C10H27N4P1}

0,1

N,-0.2923217009,0.1131858827,-1.1581358384

$\mathrm{P},-0.1706945813,0.2703963018,0.3742478147$

$\mathrm{N}, 1.3085697502,0.2644065327,1.236262178$

N,-1.0097979302,-0.9329873127,1.2576019803

$\mathrm{N},-0.7565292287,1.8206712893,0.7493104494$

C,-2.209005354,-1.5190935497,0.662722841

C, $-0.9940118368,-1.0589388997,2.7125752402$

C, $2.0294028412,-0.9926448623,1.4383588746$

C, $2.2187075675,1.4001073352,1.0819424505$

C, $-1.0027897519,2.2151615736,2.1307065703$

C, $-1.5227630615,2.5925235613,-0.2245271407$

C, $0.325669371,-0.6626105015,-2.2227085421$
$\mathbf{H P}_{\mathbf{1}}(\mathbf{d m a}) \mathbf{H}+$
dma3+.log.acs

Brutoformula: C6H20N4P1(1+)

1,1

$\mathrm{H},-2.1338933039,-1.0546676507,-0.6228413659$

$\mathrm{P}, 0.2749140744,0.2887922622,-0.2514623893$

N,-1.261908775,0.8662053716,-0.4025873845

$\mathrm{N}, 1.1867736118,1.4192085187,-1.0467598677$

$\mathrm{N}, 0.7834362097,0.1491253832,1.3134341081$

$\mathrm{N}, 0.4582437969,-1.2174461302,-0.9003450805$

C, $0.1759914178,-2.4617459562,-0.161600557$

C, $0.6064903356,-1.4138727614,-2.3518214104$

C, $2.1302140291,-0.3622975648,1.6294658934$

C, $0.1721606676,0.9312164532,2.4006311408$

C,-2.4277240248,-0.0335006462,-0.3894606772

C,-1.6147694309,2.2934853066,-0.2633303428

$\mathrm{H},-0.7160297443,-2.9547237568,-0.5603801899$ $\mathrm{H}, 0.0226282529,-2.2562620298,0.89542745$

H,1.0231094739,-3.1443267562,-0.2675474804

H, $-0.336187805,-1.7294013786,-2.8098147729$

$\mathrm{H}, 1.3580563366,-2.1863496449,-2.5300374213$

$\mathrm{H}, 0.9463596363,-0.5002577883,-2.8365267167$

$\mathrm{H}, 0.7198894728,1.8602441247,2.5900695519$

$\mathrm{H}, 0.1822129695,0.327800566,3.3107916223$

$\mathrm{H},-0.8637117298,1.1691313805,2.1677828594$

H, 2.8204138797,0.4589713188,1.8512864262

H,2.5261057488,-0.9455535136,0.7986658109

H,2.0729201014,-1.0114062706,2.5063522414

H,-2.195701695,2.6076980842,-1.1341264515

H,-0.7199091751,2.9081450635,-0.198750368

$\mathrm{H},-2.2205597456,2.4530789776,0.6336403506$

$\mathrm{H},-3.1378764449,0.2977773301,-1.1503200751$

H,-2.9275191009,-0.0223149377,0.5842122533

$\mathrm{H}, 2.1739550409,1.4992221024,-0.8450502438$

$\mathrm{H}, 0.9219159207,1.7640245426,-1.9589969153$

$\mathrm{HF}=-801.0065274 \mathrm{NImag}=0$

t-BuP $\mathbf{1}(\mathrm{dma}) \mathrm{H}^{+}$

tbup1+1.log.acs

Brutoformula: C10H28N4P1(1+)

1,1

H,-0.6526804661,1.0412022596,-1.3884791232

$\mathrm{P},-0.3317277103,0.0820396438,0.5995777096$

C,-2.1034790359,-1.184327541,2.2534252453

N,-1.4327052044,-1.1054885554,0.9433733468

$\mathrm{H}, 3.1322579861,0.0049553421,0.727363708$

$\mathrm{N},-0.0824747803,0.2886712524,-1.0188071388$

H,-1.2209847676,-3.1617751841,0.4244714318

$\mathrm{H},-1.3986158139,-2.0450599412,-0.9476860007$

C,-1.7148447606,-2.2507010992,0.0705456246

C, $2.3080212622,0.6237029214,1.0939240982$

H,2.6283349772,1.104404167,2.0231216847

C, $0.716087634,-0.3704212095,-2.1252722899$ 
C, $1.7780532837,-0.1975411323,-2.4668288196$ C, $-0.4935797091,-0.3951704017,-3.5024454621$ C, $0.3246914551,-2.182593693,-1.9473194919$ H,-0.4899851561,0.6728623684,-3.734529609 $\mathrm{H},-1.5319887811,-0.7047439377,-3.3587334863$ $\mathrm{H},-0.0834157822,-0.9389326157,-4.3596457833$ $\mathrm{H}, 0.76616778,-2.7310207056,-2.7860297482$ H, $-0.6939890029,-2.5513903416,-1.8045121564$ H,0.9010384578,-2.4220536926,-1.0494515457 H,2.2179317942,-0.7052659263,-3.3318954881 H,2.4074411322,-0.4099609936,-1.5981447827 H, $1.8006813652,0.8798191873,-2.6484362568$ $\mathrm{H},-1.2606756906,3.6539626354,-0.1373446522$ $\mathrm{H},-2.6064606287,2.4952211363,-0.0617673222$ H,-1.2870141936,2.2406150097,-1.2255192862 H,-0.8418685979,3.2943511456,2.2330906345 $\mathrm{H},-0.3121157417,1.7117899799,2.8064788636$ $\mathrm{H},-2.031818784,2.0002972156,2.455596096$ H,2.8331401604,1.495203924,1.9840804794 H,1.6526911091,2.319707886,0.9466052676 H,2.8925272871,1.2765938895,0.2228007215 H,2.5970084397,-0.9425010709,2.3746917332 H,2.7421008137,-1.1948586264,0.6263746132 $\mathrm{H}, 1.3349239074,-1.8282065095,1.5006800137$ $\mathrm{H},-0.0955283244,-0.6098313502,3.1303376588$ H,-1.0071929147,-2.1203143782,2.9901513344 $\mathrm{H},-1.8703955444,-0.584528349,3.1770247356$ H,-2.2373629148,-2.5969637305,0.8619378724 H,-2.2036971745,-1.3606724501,-0.4131822484 H,-3.1257441287,-1.0740518234,1.0775792337

$\mathrm{HF}=-957.8684499 \mathrm{NImag}=0$

\section{$\mathrm{PhP}_{1}(\mathrm{dma})_{2} \mathrm{Me}$}

phme10.log.acs

\section{Brutoformula: C11H20N3P1}

0,1

$\mathrm{N}, 0.638857212,-0.2144071749,-1.7556196714$ $\mathrm{P}, 0.2673463175,0.7118561192,-0.3902580227$ C, $1.8833878864,1.2772739643,0.263007122$ $\mathrm{N},-0.6823309344,0.0058511802,0.6394610121$ $\mathrm{N},-0.4511909835,2.1269482793,-1.0041838295$ C,- $0.4725987205,-1.0191652172,1.5499244485$ C, $-0.2032372928,-3.0817981319,3.4826388667$ C, $0.6056309421,-1.9294569616,1.5105364086$ C,-1.4172898013,-1.1869885061,2.5851226723 C, $-1.2828735626,-2.1960785506,3.5298120476$ C, $0.7363142654,-2.9374224931,2.4644152406$ $\mathrm{H}, 1.3432904243,-1.8511491987,0.7180118659$ $\mathrm{H},-2.2556032937,-0.5007713002,2.6222133166$ H,-2.0282598058,-2.2937374653,4.3126514739 H, 1.5795987076,-3.6182101716,2.4044894581 H,-0.1007605895,-3.8681610112,4.2213568247 C,-0.0813268326,-1.4508160155,-2.0498337662 C, $1.3954169227,0.3267720299,-2.8800031018$ C,-1.663621984,1.9196656558,-1.8060157572 C,-0.6024678499,3.2587435966,-0.0820724294 H,0.6268811648,-2.245477986,-2.3099694947
C, $1.710070009,0.6744617542,-2.6601968643$ C, $-0.265595374,-0.7539138579,-3.245080128$ C,1.470589729,-1.6086147949,-1.6305547694 $\mathrm{H},-0.8269867761,0.1179246355,-3.5942246711$ H,-0.9816788923,-1.5123759092,-2.9229780799 H, $0.2826624305,-1.1543788864,-4.1006775707$ H,2.0092078924,-2.0450413715,-2.473990467 $\mathrm{H}, 0.8021279304,-2.3781227814,-1.2408780909$ H,2.2051684108,-1.3633552333,-0.8639475225 H,2.2547687409,0.2711508246,-3.5168590576 H,2.4398417386,0.9582620585,-1.9005615382 $\mathrm{H}, 1.1907608558,1.5770348,-2.9943637119$ $\mathrm{H}, 2.1002901166,1.3967093693,0.3567370183$ $\mathrm{N},-1.0401604451,1.4646611524,1.1937158103$ C, $1.3126195239,-1.2326900617,2.3693806525$ H,2.2098901401,-1.8122440666,2.1366165785 $\mathrm{H}, 0.4685078077,-1.9163633759,2.4017513186$ $\mathrm{H}, 1.4387258946,-0.7753638471,3.3557099201$ $\mathrm{H},-1.8457244132,-0.3269451956,2.8716389044$ H,-1.819630776,-2.1002522997,2.7810148293 $\mathrm{H},-3.1873226901,-1.1934300648,2.1072533072$ $\mathrm{H},-2.7937936279,-2.425095759,0.058182824$ $\mathrm{N}, 1.119731977,-0.210913776,1.3300908737$ C, $-2.3541856909,1.8932149941,0.6866234682$ C,- $0.2876162148,2.5293089673,1.8709633674$ $\mathrm{H},-2.2571132207,2.6836034442,-0.0675656224$ H,-2.9506455876,2.2849344411,1.514317797 H,-2.8912282218,1.0510732508,0.250289424 H,0.0588191398,3.3016484045,1.1747868912 $\mathrm{H}, 0.5660488462,2.1186748172,2.4049908144$ H,-0.9438210461,2.9996537293,2.6067306735

$H F=-958.2924669$ NImag=0

$\mathrm{PhP}_{\mathbf{1}}$ (dma) $)_{2} \mathrm{MeH}+$

phme1+.log.acs

Brutoformula: C11H21N3P1(1+)

1,1

N,-0.7863652866,-0.945701577,-1.5609716806 $\mathrm{P}, 0.0301885711,-1.1916017275,-0.1426202966$ C, $-0.0305374265,-2.913708119,0.4306962352$ $\mathrm{N},-0.7527674361,-0.3646561522,1.0720662452$ $\mathrm{N}, 1.6001496471,-0.784474997,-0.4521443911$ C, $-0.7227513343,1.0415309774,1.3621804435$ C, $-0.6914195112,3.7583879917,2.0073957568$ C, $-0.7654498582,1.9986714979,0.3477976526$ C, $-0.6728050871,1.4428446527,2.6996504193$ C,-0.6682182016,2.7974449884,3.0173048459 C,-0.736137105,3.3538823307,0.676281426 $\mathrm{H},-0.8361157262,1.6991613905,-0.6900205703$ H,-0.6382727274,0.7001355119,3.4900756696 $\mathrm{H},-0.6384740939,3.1002173314,4.0571597236$ $\mathrm{H},-0.7674258136,4.0928197617,-0.1156813611$ $\mathrm{H},-0.6815266959,4.8120792066,2.2573305074$ C,-2.2386011039,-0.6859550282,-1.5867130494 C, $-0.2778358086,-1.5209391483,-2.8226365312$ C, $1.9767571718,0.3946793272,-1.2588646293$ C,2.6822174644,-1.2305642618,0.4467790392 H,-2.8022091652,-1.6099699823,-1.7536699375 
H,-0.772718494,-1.3205369707,-2.8944147304 H,-0.6546852597,-1.766571732,-1.1803868451 H,2.166645593,-0.3846999518,-3.1973776676 H, 1.8829020469, 1.2617775413,-2.6069250206 $\mathrm{H}, 0.7442683754,0.5305681718,-3.741409029$ $\mathrm{H},-1.389995067,3.0862492938,0.6636880724$ $\mathrm{H},-0.857332742,4.150576951,-0.6613377868$ H,0.332887993,3.4613130819,0.4392013062 $\mathrm{H},-2.5406602079,1.6998222892,-1.1835439256$ H,-1.5190259268,1.0935107798,-2.5021742162 $\mathrm{H},-1.860802716,2.8246739778,-2.3877908933$ H, $2.4204047644,1.902548453,-0.4514163898$ H,2.4812978198,0.3941275997,0.4895481397 H,1.7316516268,1.8331698719,1.1886543026

$\mathrm{HF}=-937.0048723 \mathrm{NImag}=0$

\section{t-BuP $\mathbf{P}_{1}$ (pyrr)}

pyr31.log.acs

\section{Brutoformula: C16H33N4P1 \\ 0,1}

N,-0.5429258503,-0.2518070323,-1.6453179769

$\mathrm{P},-0.1310849315,-0.0541842769,-0.02511034$

$\mathrm{N}, 1.5675995539,0.1097513042,-0.0971400883$

$\mathrm{N},-0.8561141922,-1.203494367,0.7199031828$

$\mathrm{N},-0.5523882315,1.5159129575,0.4658079441$

C, $-0.7896550547,-1.8421881493,2.0244755803$

C,- $0.270167782,0.7457724036,-2.6904444432$

C,-1.2265881338,-1.4410468045,-2.1887856586

C,-1.9798439692,1.7808830107,0.7723242082

C, $0.1241246272,2.7725826205,0.0688878871$

C, $2.3271425011,-0.8782988182,-0.9072865427$

C, $2.3818596244,0.5383567513,1.0558831484$

$\mathrm{H}, 0.7712127503,1.0846400424,-2.6655061786$

$\mathrm{H},-0.9079311135,1.6324831372,-2.5717516277$

C, $-0.6054850156,0.0133239675,-3.9990308404$

$\mathrm{H},-0.5335671133,-2.2892231489,-2.2784132087$

C,-1.6977667607,-0.9776326432,-3.5726281479

$\mathrm{H},-2.0372441298,-1.7499420453,-1.5305012786$

H,2.2123966088,-0.0899602094,1.9418346982

H, $0.2734952306,-0.5317831424,-4.3577052737$

H,-0.9192770718,0.698488542,-4.7897132556

H,2.1579052024, 1.5686878473,1.3337014567

$\mathrm{H},-2.6603608826,-0.4627550916,-3.4878642056$

$\mathrm{H},-1.8215237414,-1.8086441847,-4.2704872519$

$\mathrm{H}, 0.7585053356,3.1370032546,0.8880595636$

C,-1.0159259298,3.7670603024,-0.1811110308

H,0.7666999741,2.6272122699,-0.7996713032

H,-2.2732118045,1.3125423858, 1.7122575838

$\mathrm{H},-2.6296153809,1.3745871766,-0.0136705071$ C, $-2.0811913597,3.3110185276,0.8238576465$ $\mathrm{H},-0.7023337572,4.8043742857,-0.0421036572$ $\mathrm{H}, 1.8429156382,-1.8594731454,-0.9137835571$ H,-1.3928181112,3.6633891935,-1.2038461957 C,3.8164211098,0.3557740012,0.5558666464 $\mathrm{H},-1.8286357587,3.6722484987,1.826007864$ $\mathrm{H},-3.086020567,3.665645675,0.5850656754$ C,3.7204122806,-0.946854289,-0.2513265509
$\mathrm{H},-2.4538928844,0.0036905411,-2.4058444681$ H,-2.5678328997,-0.2294766687,-0.6564897031 $\mathrm{H},-0.8012789324,-2.4518041593,-3.0642241668$ $\mathrm{H}, 0.7897143601,-1.7225803426,-2.7568412613$ H,-0.4456763717,-0.8086786676,-3.633622329 H,2.9039534767,-0.4746408113,1.2062363058 H,3.5794363079,-1.4030483276,-0.1506884814 $\mathrm{H}, 2.4297975973,-2.1659424819,0.94210835$

H, 1.1578381627,0.696982149,-1.9063060365 $\mathrm{H}, 2.8351605692,0.1298663838,-1.8805818008$ H,2.2478500753,1.2351394005,-0.6146153089 $\mathrm{H}, 0.4741289618,-3.5521553018,-0.2959495271$ $\mathrm{H},-1.0791638149,-3.2148993714,0.4867692864$ $\mathrm{H}, 0.4291442119,-3.049111989,1.4098972911$ H,-1.0815792929,-0.9376243267,1.8387563305

$\mathrm{HF}=-937.4162286 \mathrm{NImag}=0$

\section{t-BuP $\mathbf{1}\left(\right.$ pyrr) $\mathbf{H}^{+}$ \\ pyr3+1.log.acs}

\section{Brutoformula: C16H34N4P1(1+) \\ 1,1}

N,1.8088177437,-0.0371853367,-0.2005311419 $\mathrm{P}, 0.1594013755,-0.1069928827,-0.0733636195$ N,-0.3820664372,-1.6620527027,0.0484365523 $\mathrm{H}, 0.9869846777,-4.0463912911,0.8143241449$ N,-0.3610679753,0.650079491,1.2962703067 N,-0.3055305303,0.600029881,-1.4969498409 C,2.6885510243,-0.9179927015,0.6149748472 C, $2.5964492987,1.0689679721,-0.8250610926$ C, $-1.6588562953,0.4466386437,2.0083148112$ C, $0.3438555062,1.8559326289,1.8202821233$ C, $-0.336919451,-2.5619393358,-1.1447899685$ C, $-0.4287348276,-2.4733367825,1.3041144613$ $\mathrm{H}, 2.4558237922,-1.9691441183,0.4373799323$ H,2.5642720388,-0.7135378019,1.6854528352 C, $4.0992990565,-0.5407905872,0.15106533$ H,2.6708148134,0.9090659044,-1.9059936667 C,3.9751663926,0.9554228486,-0.163706975 H,2.1275269777,2.0395589771,-0.6593250611 $\mathrm{H},-1.4375957158,-2.4354494515,1.7235049337$ $\mathrm{H}, 4.3585568064,-1.1001691819,-0.7521666827$ $\mathrm{H}, 4.8510870584,-0.7608387819,0.909750939$ $\mathrm{H}, 0.2667621996,-2.0926622341,2.0530807544$ $\mathrm{H}, 4.000223197,1.5398551522,0.7606427082$ $\mathrm{H}, 4.7690836448,1.3263075831,-0.8130142905$ H, $1.4040737278,1.6463186722,1.9677967659$ C,- $0.3881807587,2.1555678753,3.1321333676$ H,0.2497152656,2.6950682354,1.1226069492 H,-1.5902875351,-0.4258766513,2.6615005475 H,-2.479900861,0.2829926064,1.3094180296 C,-1.8305388887, 1.7263428187,2.8359121177 $\mathrm{H}, 0.0305082152,1.5526530699,3.9426934087$ H,-1.0461946494,-2.2322066557,-1.9029183401 $\mathrm{H},-0.3016023656,3.2051893594,3.4149696365$ C,-0.0962861266,-3.8955438521,0.84206297 H,-2.4193836392,1.5470084832,3.7362781524 $\mathrm{H},-2.3422613058,2.4954877031,2.2512211858$ C,-0.6789084431,-3.9423858193,-0.5752344773 
H,2.3904587745,-0.5345202778,-1.9453233729 H,4.5177058687,-1.0573780449,-0.9891680777 H,3.7751032997,-1.8051841622,0.4245946484 $\mathrm{H}, 4.5411389676,0.3044545823,1.3715237462$ $\mathrm{H}, 4.0938127908,1.1919204372,-0.0936789255$ C, $-0.6966825245,-0.8452672257,3.2008104308$ C, $0.4113585354,-2.8127049305,2.0911509321$ C, $-2.0867213151,-2.6629989567,2.1854808765$ H, $0.2053134228,-0.2311489198,3.1327032673$ H,-0.6729870916,-1.3736702919,4.1597920256 H,-1.5609398278,-0.1767742804,3.2081004426 H,1.3563907758,-2.269438287,2.0034463397 H, $0.3557242839,-3.5312499881,1.2694690225$ $\mathrm{H}, 0.4261146401,-3.3668671266,3.0361085061$ H,-2.9573440962,-2.0035333,2.1414203573 H,-2.1039089776,-3.2008130905,3.139279153 $\mathrm{H},-2.1735513162,-3.3892769436,1.3735566617$

$H F=-1190.1868279 \quad$ NImag $=0$

\section{HP $\mathbf{P}_{1}$ (pyrr)}

pyr3.log.acs

\section{Brutoformula: C12H25N4P1 \\ 0,1}

$\mathrm{N},-0.3973434587,-0.581751554,-1.3414016204$ $\mathrm{P},-0.4585392402,-0.6917988975,0.3307582561$ N,1.0856699931,-0.7092637032,1.0161622445 $\mathrm{N},-1.3319766675,-1.9646534152,0.6197238922$ $\mathrm{N},-0.9501503607,0.8780888807,0.7842255375$ H,-1.4564282705,-2.1933908062,1.599345705 C, $0.4633776572,0.3615726215,-2.0715037686$ C,-1.0570450574,-1.5375489381,-2.2522144044 C,-0.9914196022,1.2384641556,2.2259422069 C,-2.1495110262,1.4270909862,0.1011231392 C, $1.8342223904,-1.98785363,1.0686749467$ C, $2.0027435282,0.4403742775,1.1789734018$ H, 1.5001099536,0.3240407289,-1.7202333585 H, $0.1129501989,1.3941394935,-1.9426452556$ C, $0.3565496001,-0.0879641186,-3.5415169791$ H,-0.516480152,-2.4921182875,-2.2894839881 C,-0.9931001707,-0.8185843866,-3.6031075851 H,-2.069370722,-1.7524673155,-1.9129822916 H,1.9228530342,0.8529026137,2.1932475322 H,1.166454098,-0.7849540979,-3.7781330215 $\mathrm{H}, 0.4298679788,0.7520757972,-4.2354452934$ $\mathrm{H}, 1.7650169306,1.24379927,0.4809417782$ H,-1.8126060708,-0.0970651979,-3.682473614 H,-1.0675089189,-1.5077950908,-4.4472339052 $\mathrm{H},-1.8771241195,1.8744880223,-0.8576987159$ C,-2.7124856256,2.4569518591,1.0873598399 $\mathrm{H},-2.8902362515,0.6408088849,-0.0913367619$ $\mathrm{H},-0.2147614633,1.9800607192,2.4475349408$ $\mathrm{H},-0.8043927429,0.3687135806,2.8615335534$ C,-2.3866856774,1.8355565166,2.4509889407 H,-2.1943894199,3.4148988501,0.9740635212 H, $1.359833675,-2.6939983404,1.7527654947$ H,-3.7794260284,2.6320992691,0.9339630662 C,3.3970460349,-0.1585509551,0.9637360721
$\mathrm{H}, 0.6657584299,-2.5630552751,-1.5880455816$ H,-0.2662996674,-4.7460644378,-1.1860034741 $\mathrm{H},-1.7636419831,-4.0744001412,-0.5350794958$ $\mathrm{H},-0.5203843872,-4.6482048739,1.5079479255$ C,-1.6013433391,1.2089239525,-1.9642670605 $\mathrm{H}, 0.3500762652,0.4363831405,-2.2496293911$ C,-2.7660891927,0.213264362,-1.851986249 C,-1.3740318824,1.5704480309,-3.4400354671 C,-1.9032332407,2.4874062219,-1.1686588805 $\mathrm{H},-2.5977379509,-0.6626541823,-2.4819049194$ H,-3.693972657,0.6866895157,-2.1810701563 H,-2.9154884071,-0.1275078212,-0.8251835217 $\mathrm{H},-1.1466927724,0.6813021609,-4.0357621093$ $\mathrm{H},-0.5554074417,2.2868967966,-3.5495577954$ H,-2.2743287507,2.0258125104,-3.8562047095 H,-2.7969898593,2.9706558779,-1.569837384 $\mathrm{H},-1.0736920894,3.1942903122,-1.2406241623$ H,-2.0891580766,2.2758221125,-0.1152302219

$\mathrm{HF}=-1190.6244126 \mathrm{NImag}=0$

$\mathrm{HP}_{1}$ (pyrr)H+

pyr3+.log.acs

Brutoformula: C12H26N4P1(1+)

1,1

N,-0.4080025941,-0.5846470977,-1.4580050541 $\mathrm{P},-0.2775568025,-0.6954417624,0.1754633594$ N,1.2560173937,-0.4596395992,0.7186242311 H,3.8470348546,0.8394659229,0.348985123 $\mathrm{N},-1.3317573693,0.4026523759,0.8019169573$ $\mathrm{N},-0.6357341423,-2.2399660648,0.6703419952$ C, $0.7409283074,-0.8544070443,-2.3801019691$ C,-1.6992294831,-0.6331106843,-2.2010497243 C,-1.8602369779,0.2714395149,2.1925498983 C, $-1.5482134638,1.7873053274,0.2825568855$ C, $2.3462971933,-1.4760948161,0.7829288624$ C, $1.786765057,0.9029687625,1.0307456887$ H,1.3214188044,-1.7210804411,-2.05855393 H, $1.4010561715,0.0161688133,-2.4013893706$ C,0.0741524289,-1.0970518089,-3.7441116366 H,-2.1628378598,-1.6241603436,-2.1176447867 C,-1.2699241834,-0.3637557759,-3.6437850038 $\mathrm{H},-2.3995006339,0.1054042126,-1.8131622766$ H, $1.2894724374,1.2939821221,1.9221682157$ $\mathrm{H},-0.0934889357,-2.1670252645,-3.8933530627$ $\mathrm{H}, 0.6965970239,-0.7417683615,-4.5658182522$ H,1.6166700594,1.6014700981,0.2078682522 H,-1.1375043228,0.7106711315,-3.7984138489 H,-2.006649779,-0.7212276866,-4.3640325707 $\mathrm{H},-0.6504832608,2.185966967,-0.1902828166$ C,-1.970949625,2.5803781397,1.5236840666 $\mathrm{H},-2.3485394342,1.779962746,-0.462995962$ $\mathrm{H},-1.0397536505,0.2438001438,2.9193393274$ H,-2.4459725567,-0.6434014899,2.2957785564 C,-2.7054238836,1.5369020785,2.3752028272 $\mathrm{H},-1.0902382796,2.9530524383,2.0547098369$ H, 1.9727006863,-2.4197370487,1.1763864582 $\mathrm{H},-2.5924765873,3.4384066366,1.2648696277$ C,3.288273944,0.6729026443,1.2736826411 
$\mathrm{H},-2.4047681676,2.5550562034,3.2727692775$ H,-3.1030510955,1.0407868208,2.6803557809 C,3.2424338968,-1.5794205335,1.5244313464 $\mathrm{H}, 1.867052886,-2.4637090141,0.0804366521$ $\mathrm{H}, 4.0078223038,-2.2726778766,1.169650942$ H,3.2935034677,-1.5568015144,2.6177686588 $\mathrm{H}, 4.1817156373,0.4201345926,1.4565742423$ H,3.6295770438,-0.1997364684,-0.1056404049

$H F=-1032.9040286$ NImag $=0$

\section{$\operatorname{EtP}_{1}$ (pyrr)}

pyr3et.log.acs

\section{Brutoformula: C14H29N4P1}

0,1

$\mathrm{N},-0.5352157642,-0.9338306451,-1.1173612789$

P, $-0.2620168288,-0.7069884559,0.5210285789$

$\mathrm{N}, 1.3821883559,-0.2414626484,0.5791601494$

$\mathrm{N},-0.7848524682,-2.0011331179,1.2166369314$

$\mathrm{N},-0.9758508695,0.7266427118,1.067798213$

C, $-0.7552797582,-2.3119179766,2.6351036186$

C, $-0.3625546129,0.1103046909,-2.1390423822$

C,-0.9488574477,-2.2220833006,-1.7120791968

C, $-2.4021989701,0.7014256744,1.4837443067$

C, $-0.5592332977,2.1057407813,0.7393040004$

C, $2.3569346552,-1.0249798586,-0.2267206114$

C, $2.0000938072,0.125105663,1.8725149858$

$\mathrm{H}, 0.5660016318,0.6703482911,-1.990097917$

$\mathrm{H},-1.1915453346,0.8313592293,-2.1154782273$

C, $-0.3731168466,-0.6687948403,-3.4614559454$

$\mathrm{H},-0.1248924505,-2.9470552601,-1.6883928933$

C,-1.3083556377,-1.8478344869,-3.1565155787

$\mathrm{H},-1.7782627875,-2.6562855696,-1.1551328734$ H,1.8084483799,-0.6278364097,2.649652411

$\mathrm{H}, 0.6330512231,-1.0360874601,-3.6874721808$ H,-0.7035101135,-0.0553520789,-4.302675899 H,1.6122152676,1.0782724542,2.236552044 H,-2.3520309136,-1.5215722633,-3.211947646 H,-1.1828406699,-2.6864392012,-3.8447086377 $\mathrm{H}, 0.4687876861,2.2927339562,1.050391138$ C,-1.5758910782,2.9697485532,1.4906768838 $\mathrm{H},-0.6171993766,2.3082236117,-0.3381468676$ H,-2.4854747686,0.3537423433,2.5184631251 $\mathrm{H},-2.9888945894,0.0197866868,0.8627924625$ C,-2.8715640185,2.1597447952,1.3485098583 H,-1.2910666442,3.0500004063,2.5446197843 $\mathrm{H}, 2.009750709,-2.0467608042,-0.406007941$ $\mathrm{H},-1.6484566193,3.9799770008,1.0814207959$ C,3.493552155,0.1747563527,1.5471445399 $\mathrm{H},-3.6293763896,2.4196963461,2.0904263047$ H,-3.3061937298,2.3267006256,0.357794316 C,3.6617271927,-1.0167337389,0.5943602216 H,2.4972846007,-0.5464415543,-1.2020236599 $\mathrm{H}, 4.5473036151,-0.9408988748,-0.0400202384$ $\mathrm{H}, 3.7470588421,-1.9419986586,1.1717354144$ H,4.1191026013,0.1042822624,2.4398165356 H,3.7338292599,1.1131742945,1.0372789813 H,0.2311030235,-2.6974952154,2.9388267722
H,-2.7906145474,1.8195292533,3.4248746335 H,-3.7149126637,1.3721719915,1.988860511 C,3.3803020662,-0.8023108634,1.6853477983 $\mathrm{H}, 2.7638920389,-1.6612414958,-0.2125600474$ $\mathrm{H}, 4.3776831693,-1.2214857436,1.5477292812$ H,3.1017876048,-0.9292907109,2.7353376798 $\mathrm{H}, 3.683490471,1.3556175372,2.0263794042$ H,-0.5285191072,-2.5011958703,1.6416248904 H,-1.3858804885,-2.7328949428,0.2047939154

$$
\mathrm{HF}=-1033.335283 \mathrm{NImag}=0
$$

\section{EtP $\mathbf{P}_{1}$ (pyrr) $\mathrm{H}^{+}$}

pyr3+et.log.acs

\section{Brutoformula: C14H30N4P1(1+)}

1,1

$\mathrm{N},-0.5738600894,-0.6176827309,-1.3675799117$ P,- $0.4594646372,-0.6243257877,0.275321415$ N,1.0979663637,-0.5101533927,0.7976858017 $\mathrm{H}, 3.8333844868,0.5677552581,0.6402415212$ $\mathrm{N},-1.3593719003,0.6542184368,0.7977854182$ $\mathrm{N},-1.0218113253,-2.0688703952,0.8556341797$ C, $0.5437248482,-1.0098705749,-2.2796067842$ C, $-1.862481951,-0.5731849873,-2.108477978$ C, $-2.0805120731,0.6725799684,2.1027687404$ C, $-1.3433774225,2.0135655523,0.1785919492$ C,2.0918245243,-1.6173265441,0.8223261227 C, $1.7421723973,0.7794002282,1.1897828201$ H,1.0095104543,-1.9479168021,-1.969870037 H, $1.3068725327,-0.2279306807,-2.2774853911$ C,-0.1300431296,-1.1487866667,-3.6581982019 H,-2.391275843,-1.5328940173,-2.0397479991 C,-1.4133831899,-0.3136398627,-3.5463279335 H,-2.513797688,0.2025362633,-1.7064887267 $\mathrm{H}, 1.231807108,1.1959666631,2.0614482556$ $\mathrm{H},-0.3815278061,-2.1957530615,-3.846709923$ H, $0.5272116529,-0.817914357,-4.4627847454$ H, $1.6981080559,1.5143499233,0.3827727231$ H,-1.1975678002,0.7497334048,-3.6821764538 $\mathrm{H},-2.1730833609,-0.6010340231,-4.2741599291$ H,-0.3454883136,2.2934285921,-0.1600637025 C,-1.8644037563,2.9273767062,1.2939053227 $\mathrm{H},-2.0099795774,2.0351822495,-0.6885780793$ $\mathrm{H},-1.3769877911,0.625836665,2.9417623932$ $\mathrm{H},-2.7644512137,-0.1735899828,2.1763273873$ C, $-2.8135275953,2.0178962678,2.0837296267$ H,-1.0377768454,3.2579173197,1.9294530274 H,1.6199866967,-2.5528517721,1.1183827575 H,-2.3539045747,3.8163709185,0.8944713192 C,3.1976998898,0.3913514668,1.511721242 H,-3.0208528679,2.382182664,3.090454792 $\mathrm{H},-3.7680172359,1.9172304996,1.5596441172$ C,3.134872326,-1.1112019011,1.8189295076 $\mathrm{H}, 2.5416731355,-1.758680875,-0.1664772677$ H,4.0966692178,-1.6109670228,1.6972530386 H,2.7911831788,-1.283570899,2.8429331408 H,3.5981018073,0.9817338848,2.3363160367 C, $-0.9677888712,-2.5827416095,2.2417309747$ H,-1.6948783627,-2.5345465689,0.2593891435 
C,-1.800239176,-3.3795216111,2.9681382761 H,-0.9424586909,-1.4314163328,3.2730367977 H,-1.6219948125,-4.2798433346,2.3738690789 $\mathrm{H},-2.8057515363,-3.0186186341,2.7340254315$ H,-1.7645191961,-3.6494210767,4.0294079904

$\mathrm{HF}=-1111.5381274$ NImag $=0$

\section{2-Cl- $\mathrm{C}_{6} \mathbf{H}_{\mathbf{4}} \mathbf{P}_{\mathbf{1}}$ (dma)}

c2lph.log.acs

Brutoformula: C12H22C11N4P1

0,1

$\mathrm{N},-0.3327045694,-0.2239987837,-2.2563473958$ $\mathrm{P}, 0.020974941,0.1619372865,-0.6571721806$ $\mathrm{N}, 1.6545977754,0.5556493381,-0.4027892307$ N, $-0.4563278836,-1.0721644316,0.178076382$ N,-0.705497252,1.6883451409,-0.4897704492 C, $0.0473065386,0.65805613,-3.3543012792$ C, $-0.6567997801,-1.5895836563,-2.6717021587$ C, $-2.1124303174,1.8186669175,-0.888282917$ C, $-0.3874749256,2.5427228385,0.6598581344$ C, $2.5775533623,-0.5256027152,-0.0458147822$ C, $2.3251322776,1.671745114,-1.0697831957$ $\mathrm{H}, 0.9898291358,0.3489954308,-3.8274696417$ $\mathrm{H}, 0.1490035331,1.6864344749,-3.0097213213$ $\mathrm{H},-0.7334517605,0.6388984629,-4.1229094588$ H,0.2064375687,-2.0838255923,-3.1380166805 $\mathrm{H},-1.4704405131,-1.5585932818,-3.4055327453$ $\mathrm{H},-0.9748496555,-2.1660992063,-1.8073866979$ H,- $1.032614846,2.3240480858,1.5201213587$ $\mathrm{H},-0.5364245639,3.5907745094,0.3788484121$ $\mathrm{H}, 0.6489845582,2.4112073307,0.9625078947$ H,-2.7932744841,1.5464102173,-0.071399897 $\mathrm{H},-2.324845322,1.1791148265,-1.742696453$ H,-2.3094054467,2.8580721691,-1.1695736928 H,3.0873728618,2.088664134,-0.4019680135 H, 1.6149238646,2.4608023272,-1.3094006962 H,2.8268294755, 1.3573232265,-1.9953813334 H,3.0724423121,-0.948631215,-0.9312922766 $\mathrm{H}, 2.0471259633,-1.3231705884,0.4696123558$ H,3.3516293828,-0.1341261786,0.62235187 C, $-0.5420174766,-1.3517174935,1.5176542528$ C,- $0.7843801489,-2.0725564464,4.2706533725$ C, $-1.2655543312,-2.493325323,1.9445040201$ C, $0.0630112668,-0.5991969712,2.5521285955$ C, $-0.0562050157,-0.9465331397,3.8930882933$ C, $-1.3884730745,-2.846683321,3.2813587073$ $\mathrm{Cl},-2.0489413742,-3.5130530283,0.7383823217$ $\mathrm{H}, 0.6429664206,0.2742377593,2.2783795245$ $\mathrm{H}, 0.4269009496,-0.3329446537,4.6461614631$ H,-1.9589937435,-3.7297097969,3.5418801439 H,-0.8819157043,-2.350589895,5.3131453935

$H F=-1491.3147119$ NImag $=0$

\section{$\mathbf{P h P}_{\mathbf{1}}$ (pyrr)}

pyr3ph.log.acs
H,-0.0973045455,-2.1367208709,2.7271898344 C,-0.8704980604,-4.1045704074,2.2583432413 $\mathrm{H},-1.8496932321,-2.2596592625,2.8038199988$ $\mathrm{H}, 0.0252019431,-4.4552218458,1.7410158662$ $\mathrm{H},-1.7420532592,-4.5607561255,1.7799398097$ $\mathrm{H},-0.8365592742,-4.4650445628,3.2888364311$

$$
\mathrm{HF}=-1111.9762296 \mathrm{NImag}=0
$$

\section{2-Cl- $\mathrm{C}_{6} \mathrm{H}_{4}-\mathrm{P}_{\mathbf{1}}$ (dma)H+}

c2lph+.log.acs

\section{Brutoformula: C12H23C11N4P1(1+)}

1,1

N,-0.0012067337,-0.0553626153,-2.3503088077 $\mathrm{P}, 0.25883932,-0.2174505173,-0.7261548612$ $\mathrm{N}, 0.6793917726,-1.7955089118,-0.5090054195$ $\mathrm{H},-1.9801230371,0.1501635739,-0.515222323$ $\mathrm{N}, 1.3854477783,0.8421036934,-0.158816262$ C, $0.3147542745,1.1802988566,-3.0849557563$ C,-0.9311435044,-0.9531256833,-3.0609263587 C, $1.0934782065,2.2698344991,0.0682470982$ C, $2.8077970565,0.4921484879,-0.0233441776$ C, $0.2735669258,-2.58819003,0.6639827428$ C,1.6255353206,-2.4889134492,-1.4015621868 H,0.6486059882,0.910472382,-4.0891589564 H,1.1227309114,1.7218390215,-2.5983398854 $\mathrm{H},-0.5575738266,1.8363891321,-3.1717638328$ $\mathrm{H},-0.4950875989,-1.236061338,-4.0219515078$ H,-1.8904286081,-0.4588017559,-3.2484102432 H,-1.1070952974,-1.859291451,-2.4825894433 H,3.1751041085,0.8689185161,0.9345573734 $\mathrm{H}, 3.4057581678,0.9364952189,-0.8250183425$ $\mathrm{H}, 2.9448323284,-0.5867522078,-0.0368813277$ $\mathrm{H}, 1.3538149435,2.535209146,1.0956314451$ $\mathrm{H}, 0.036194503,2.479496482,-0.0824579744$ H, 1.6770170356,2.893732257,-0.6150493865 H,2.5315593999,-2.772817166,-0.8577471772 H,1.9022457597,-1.8535691376,-2.2409265087 H,1.1585516961,-3.3974994107,-1.7915339144 $\mathrm{H},-0.0526953333,-3.5737552689,0.3229994566$ H, $-0.5575517367,-2.1185919356,1.1839977049$ H,1.1059306695,-2.7187970526,1.3622336005 C,-1.4438260744,0.4558863432,1.4307541144 C,-2.1290554166,1.1409069125,4.0701011338 C, $-2.7325324283,0.9111602263,1.752986343$ C, $-0.5073149464,0.346412045,2.4637447688$ C,-0.8469406097,0.6909764501,3.7689542603 C, $-3.0761418778,1.2474884265,3.0559623557$ Cl,-3.9462382694,1.0524807295,0.4854784475 $\mathrm{H}, 0.492896712,-0.0062185547,2.2490145515$ $\mathrm{H},-0.103945142,0.5990387031,4.5518328444$ $\mathrm{H},-4.0807528685,1.5907800806,3.2669981077$ $\mathrm{H},-2.3968386342,1.4048648522,5.0853655284$ $\mathrm{N},-1.1575609349,0.1236104511,0.079282779$

$H F=-1491.7267997 \quad$ NImag $=0$

PhP $_{1}\left(\right.$ pyrr)) $H^{+}$

pyr3ph+.log.acs 


\section{Brutoformula: C18H29N4P1}

0,1

N,1.9376770218,0.3761197886,-0.3561204161 $\mathrm{P}, 0.3100372601,0.1224951349,-0.0588357224$ $\mathrm{N}, 0.2431807481,-1.5275195738,0.2915578853$ $\mathrm{N},-0.4450063671,0.7037149388,-1.3068352208$ N,-0.1413405428,0.8410776415,1.3990290464 C,-1.7533097513,0.6224737476,-1.7401855518 C,3.0641496474,-0.3767453755,0.2170166636 C, $2.413488165,1.4501103532,-1.2602581629$ C, $-1.2416959546,1.831064179,1.4909646052$ C, $0.7673825481,0.9507502192,2.558172081$ C, $0.4544065273,-2.5061881843,-0.8033524818$ C,- $0.5508960561,-2.1249134269,1.3871615851$ $\mathrm{H}, 2.8158340785,-1.4327988267,0.324344163$ H,3.3447937731,-0.0004287395,1.2117847561 C,4.1971101392,-0.1174413135,-0.7811505728 $\mathrm{H}, 2.0257350773,1.2811284331,-2.2672152937$ C,3.9478841443,1.3393145948,-1.1980170858 H,2.0620860301,2.434474638,-0.9377834352 H,-1.5850330988,-1.7610011901,1.386011967 $\mathrm{H}, 4.0903187721,-0.7838139739,-1.6426350614$ $\mathrm{H}, 5.18546364,-0.2794298781,-0.3450940749$ H,- $0.120551684,-1.8882755246,2.3628535347$ $\mathrm{H}, 4.3468016858,2.0154522474,-0.4351293484$ $\mathrm{H}, 4.4153019532,1.6020519724,-2.1490416998$ $\mathrm{H}, 1.0079291818,-0.0309186251,2.9790739655$ C, $-0.0015768714,1.8349751077,3.5484574209$ $\mathrm{H}, 1.711912527,1.4284527735,2.2686965675$ $\mathrm{H},-2.187542429,1.3344087326,1.7386659272$ H,-1.3785490507,2.3490540703,0.5414505667 C,-0.8053702521,2.763275569,2.6275832449 $\mathrm{H},-0.6805323714,1.2229608374,4.1506308796$ H,0.2124272632,-2.0655695965,-1.7728919406 $\mathrm{H}, 0.6655444394,2.3666155976,4.2303499258$ C,-0.5105488545,-3.6290075729,1.0869742334 $\mathrm{H},-1.6544714498,3.235575813,3.1257303804$ $\mathrm{H},-0.1598532142,3.5564611982,2.2369238734$ C,- $-0.4678388466,-3.6793334259,-0.4462985743$ $\mathrm{H}, 1.503387858,-2.8296219729,-0.8332699298$ H,-0.1020009893,-4.6305593878,-0.8382828763 $\mathrm{H},-1.4662302127,-3.5056177297,-0.8575726769$ $\mathrm{H},-1.3658190572,-4.1560488994,1.5149123723$ H, $0.3997876263,-4.0712846735,1.5044810384$ C,-4.3963299692,0.5446872928,-2.7946153529 C, $-2.0895897176,1.2717913899,-2.9504184147$ C,-2.7964849773,-0.0646874474,-1.0782976776 C,-4.0883237239,-0.1017973652,-1.5994669758 C,-3.3798139602,1.2329719021,-3.462308886 H,-1.3035698946,1.8047336992,-3.4733210411 $\mathrm{H},-2.5898294625,-0.57339053,-0.1430136989$ $\mathrm{H},-4.8616132351,-0.6406779942,-1.060570414$ $\mathrm{H},-3.5964441487,1.7452495823,-4.3944950572$ H,-5.4024739625,0.515629774,-3.1963490384

$H F=-1264.009253$ NImag $=0$
Brutoformula: C18H30N4P1(1+)

1,1

$\mathrm{N}, 0.1537241991,0.1178705673,-2.0214397644$ $\mathrm{P}, 0.0248705713,0.0034055384,-0.3837767699$ $\mathrm{N},-1.5451757141,-0.1300449294,0.0788962313$ $\mathrm{H},-4.2289520073,-1.1030052182,-0.4064805311$ $\mathrm{N}, 0.9306376482,-1.2749936704,0.1148058137$ $\mathrm{N}, 0.6227513104,1.4171278657,0.2647872502$ C,- $-0.9634310345,0.5025565384,-2.9376454443$ C, $1.4390915685,0.0220355463,-2.7642401872$ C, $2.0100144178,-1.2072348956,1.1521818296$ C, $0.8827638568,-2.6255393959,-0.5128765134$ C,-2.5019254411,1.0077644243,0.198900354 C,-2.2230817457,-1.432893742,0.3516159641 H,-1.4499080576,1.4251303826,-2.6153246128 $\mathrm{H},-1.7122673867,-0.2930543714,-2.9580547599$ C, $-0.277434486,0.6745870438,-4.304345319$ $\mathrm{H}, 2.0147650313,0.9517878021,-2.6750372155$ C, $0.9848118445,-0.1934211915,-4.2087468878$ H,2.0500933536,-0.7921775014,-2.3738689196 $\mathrm{H},-1.802618365,-1.88513468,1.2543978779$ $\mathrm{H},-0.0007752695,1.7215935231,-4.4547453068$ $\mathrm{H},-0.9353463437,0.3860872412,-5.1245993373$ H,-2.0957558873,-2.1349746547,-0.475178139 $\mathrm{H}, 0.741942736,-1.2473920299,-4.3712678314$ H,1.7533470892,0.0901298404,-4.9286700185 $\mathrm{H}, 0.0847553339,-3.2276427702,-0.0671216339$ C,2.2542838918,-3.2159451447,-0.1789041056 $\mathrm{H}, 0.6918253107,-2.5473105729,-1.5845662327$ $\mathrm{H}, 1.6217930537,-0.867810085,2.1120513511$ $\mathrm{H}, 2.7870808025,-0.5085889089,0.8318140588$ C, $2.5398144301,-2.647057321,1.216746073$ $\mathrm{H}, 2.2468255231,-4.3063037634,-0.2041489389$ H,-2.022574566,1.8622425263,0.6760060185 H,3.0027284454,-2.8675774532,-0.896760848 C,-3.6971650979,-1.0450678715,0.5470292737 H,1.9920539888,-3.2158529238,1.9730535733 H,3.5968278675,-2.6729754442,1.4834711855 C,-3.6388733367,0.4100763594,1.0304642092 H,-2.8656832269, $1.3164997838,-0.7865878339$ $\mathrm{H},-4.5768935954,0.9465794305,0.8830674774$ $\mathrm{H},-3.3889336589,0.4545945518,2.0940307447$ $\mathrm{H},-4.1995170863,-1.7124442285,1.2479952228$ C, $0.6833134616,1.908420454,1.6039706269$ H, 1.0991804815,2.0017608291,-0.4093043862 C, $0.8541387298,2.9799421901,4.1862592856$ C, $1.4253034937,3.0706013953,1.8393061601$ C, $0.0257338805,1.2838909042,2.6670212168$ C, $0.1174211078,1.8230822935,3.950075336$ C, $1.5059624454,3.601475348,3.122275887$ H, 1.9402728107,3.5607227722,1.0188551961 H,-0.559661413,0.3880183975,2.5036041246 $\mathrm{H},-0.3956621328,1.3303340422,4.7678183759$ H,2.0835865572,4.503100234,3.2881274655 $\mathrm{H}, 0.9199206111,3.3930249401,5.1850633527$

$$
\mathrm{HF}=-1264.4344013 \mathrm{NImag}=0
$$

2,6- $\mathrm{Cl}_{2} \mathrm{C}_{6} \mathrm{H}_{3}-\mathrm{P}_{1}$ (pyrr) $\mathrm{H}+$ 
pyr3phc121.log.acs

Brutoformula: C18H27C12N4P1

0,1

N, $0.6492983834,-1.6808342065,-0.0859765125$ P, $0.1117008516,-0.1248819003,0.2288989842$ $\mathrm{N},-1.3866004853,-0.1044903298,-0.546860251$ $\mathrm{N}, 1.1405998203,1.0137728171,-0.0767521664$ N,-0.2133619797,-0.0489724278,1.864812867 C, $1.4215999727,1.8868639406,-1.0818032853$ C, $-0.1659686691,-2.8584733434,-0.4506820493$ C, $2.0097589627,-2.0786709896,0.3364124842$ C, $0.1125953148,1.0749007411,2.7648049753$ C, $-0.8181231068,-1.1747800317,2.6024821659$ C,-1.4866218836,-0.266336564,-2.0228790655 C,-2.4251535579,0.8462416755,-0.0661106347 $\mathrm{H},-0.3104693878,-2.9022068255,-1.5370690866$ H,-1.1541010354,-2.8270780132,0.0125305245 C,0.6749513866,-4.0523777155,0.0223044582 H,2.7597051227,-1.4350487367,-0.1228548311 C,2.1159031002,-3.5405800299,-0.1133119088 $\mathrm{H}, 2.1191309263,-1.9984271276,1.4273511724$ $\mathrm{H},-1.9887778023,1.8198893468,0.1845320032$ $\mathrm{H}, 0.4772879643,-4.9531865023,-0.5628094527$ $\mathrm{H}, 0.4534358939,-4.2787524447,1.070289437$ $\mathrm{H},-2.9199078891,0.4568456808,0.8270968473$ $\mathrm{H}, 2.8342724473,-4.1063428149,0.4832751466$ H,2.4358537623,-3.5872861019,-1.1587618812 $\mathrm{H},-1.7910956215,-1.4577437058,2.1856584372$ C,-0.9447618762,-0.6542669482,4.0457098173 $\mathrm{H},-0.1705389511,-2.0584702258,2.5562158836$ H, $-0.6683438764,1.8454907485,2.7348101784$ H, $1.0499234534,1.5389067694,2.4650396936$ C, $0.1582815536,0.4098533325,4.1431758009$ H,-1.9242348652,-0.1883065605,4.191337698 $\mathrm{H},-1.8984655738,-1.2544791436,-2.2596259687$ $\mathrm{H},-0.8459676592,-1.4551175551,4.781314833$ C,-3.3825594685,0.9804183284,-1.2562742823 H,-0.0034601829,1.1215380443,4.9554732151 H,1.1325689176,-0.0647407972,4.2973150592 C, $-2.4523976794,0.8387831516,-2.4677843518$ $\mathrm{H},-0.5089610777,-0.1913832364,-2.502404505$ $\mathrm{H},-2.9778582851,0.5835559087,-3.3905152342$ $\mathrm{H},-1.9056531031,1.7712659837,-2.6354271488$ H,-3.929219169,1.9251838817,-1.2362254199 H,-4.1147430086,0.1665665758,-1.2465351038 C,2.1472406195,3.8302227053,-3.0751743316 C, $1.1381057868,3.2767883805,-0.963294335$ C, $2.0922040908,1.5556519459,-2.2908540953$ C, $2.448250665,2.4849173948,-3.259413666$ C, $1.4840437438,4.2237149088,-1.9171132652$ $\mathrm{Cl}, 0.2962326328,3.849910428,0.4760423511$ $\mathrm{Cl}, 2.5029071732,-0.1306499246,-2.6099859253$ H,2.9619308727,2.1478173469,-4.1507735466 H, 1.2333425161,5.2629139555,-1.746288231 H,2.4262202636,4.5618702094,-3.8233234965

$\mathrm{HF}=-2183.2544744 \mathrm{NImag}=0$ pyr3ph+cl2.log.acs

Brutoformula: XYZ

$\mathrm{N}, 0,-0.3136138722,-0.3812317896,-1.8575686304$ $\mathrm{P}, 0,-0.2236489747,0.078973995,-0.2762298513$ $\mathrm{N}, 0,1.2703555851,0.6585155185,0.0894904589$ $\mathrm{H}, 0,3.7491341263,0.5483758859,-1.213569375$ $\mathrm{N}, 0,-1.3950156061,1.1962520737,-0.0158771489$ $\mathrm{N}, 0,-0.5143141798,-1.3601200236,0.557845042$ C, $0,0.6934415568,-1.2857889601,-2.4889473453$ C, $0,-1.2581938425,0.1579337485,-2.8792976649$ C, $0,-2.8437379488,0.8544826977,0.0731571974$ C, $0,-1.1481406036,2.6177555614,0.3609619372$ C,0,2.2153495235,0.1145102871,1.1152532473 C, $0,1.9494694031,1.6711454899,-0.7691231074$ $\mathrm{H}, 0,0.7659659907,-2.2377381271,-1.959515785$ $\mathrm{H}, 0,1.6817256629,-0.8153357992,-2.4694894446$ C, $0,0.1842262334,-1.4523853413,-3.9268472557$ H, $0,-1.4427149593,1.2190304042,-2.715119769$ C, $0,-0.5476258001,-0.1326805244,-4.2036690899$ $\mathrm{H}, 0,-2.2141617424,-0.3728243173,-2.8284180199$ $\mathrm{H}, 0,1.7090047067,2.6830608697,-0.4288760273$ $\mathrm{H}, 0,-0.5151453324,-2.291096623,-3.9828005198$ $\mathrm{H}, 0,0.9982467859,-1.6498448621,-4.6250273681$ $\mathrm{H}, 0,1.6269981448,1.5730474453,-1.8078470955$ $\mathrm{H}, 0,0.1692182742,0.662084143,-4.4304138303$ $\mathrm{H}, 0,-1.2493983041,-0.1979539688,-5.0359887947$ $\mathrm{H}, 0,-0.6259402697,2.6668075374,1.3200993813$ C, $0,-2.559653802,3.2142556646,0.4522755426$ $\mathrm{H}, 0,-0.5427883235,3.127973192,-0.3897942444$ $\mathrm{H}, 0,-2.9783888106,-0.1053535955,0.5740424874$ $\mathrm{H}, 0,-3.2826272078,0.7888326436,-0.9274650132$ C, $0,-3.4409778134,2.0261165055,0.8594983866$ $\mathrm{H}, 0,-2.6004835464,4.0416499902,1.1616804989$ $\mathrm{H}, 0,1.8162094272,0.2316464875,2.1218407263$ $\mathrm{H}, 0,-2.8682883162,3.5976511411,-0.5241971502$ C,0,3.4349259612,1.3690725584,-0.56241178 $\mathrm{H}, 0,-3.3607022794,1.8370479196,1.9322920856$ $\mathrm{H}, 0,-4.4948296118,2.1772191949,0.6228101612$ C, $0,3.4926267421,0.9432925937,0.9096921169$ $\mathrm{H}, 0,2.3959415456,-0.9471046594,0.9298834823$ $\mathrm{H}, 0,4.3857500755,0.3679964377,1.1557944484$ $\mathrm{H}, 0,3.475234572,1.8244012078,1.5566073982$ $\mathrm{H}, 0,4.0622248852,2.2337737643,-0.7824493744$ C, $0,-0.2070828642,-1.9124057331,1.8267181541$ $\mathrm{H}, 0,-0.6945037163,-2.0965842192,-0.1160152688$ C, $0,0.3452150751,-3.2070928688,4.2837301213$ C, $0,0.2125679831,-3.2582302173,1.8895921324$ C, $0,-0.3519710668,-1.2553689259,3.0575645527$ C, $0,-0.0686284951,-1.8820126738,4.2673261894$ C, $0,0.4779788453,-3.9041082801,3.0877802895$ $\mathrm{Cl}, 0,0.3823276835,-4.1792443058,0.3988917076$ $\mathrm{Cl}, 0,-0.9062039153,0.407108142,3.1225429788$ $\mathrm{H}, 0,-0.1904427907,-1.3266031698,5.1879026406$ $\mathrm{H}, 0,0.7890604928,-4.9403280487,3.0766888903$ $\mathrm{H}, 0,0.5560247091,-3.6985760675,5.2249966967$

$\mathrm{HF}=-2183.6406 \mathrm{NImag}=0$ 
2,5- $-\mathrm{Cl}_{2}-\mathrm{C}_{6} \mathrm{H}_{3} \mathbf{P}_{1}$ (pyrr)

cl25.log.acs

cl25

Charge $=0$ Multiplicity $=1$

$\mathrm{N}, 0,-0.124021164,-1.9044247566,0.1408425734$

$\mathrm{P}, 0,0.0424910532,-0.2516449755,-0.0146967214$

$\mathrm{N}, 0,-0.7080958362,0.1009091326,-1.4854065865$

$\mathrm{N}, 0,1.5849668619,0.0014621875,0.1736090968$

$\mathrm{N}, 0,-0.933678295,0.5785297838,1.075069327$

C, $0,2.342854333,1.1346957518,0.2471688771$

C, $0,-1.4295726049,-2.5852556787,0.2067492779$

$\mathrm{C}, 0,1.0011114009,-2.8606465314,0.2619162519$

C, $0,-0.4802508234,0.6679455595,2.4889518055$

$\mathrm{C}, 0,-2.4084083424,0.7258457419,0.9945849266$

C, $0,-0.4041166107,-0.7680576594,-2.6588278748$

C, $0,-0.9269369677,1.495186938,-1.9421912999$

$\mathrm{H}, 0,-2.0869608778,-2.2800040613,-0.6137986911$

$\mathrm{H}, 0,-1.9454747266,-2.3562930998,1.1488319791$

C, $0,-1.0701457858,-4.0774667778,0.136808492$

$\mathrm{H}, 0,1.4682193003,-3.0359972477,-0.7150279383$

C, $0,0.3227230433,-4.133180621,0.7802805324$

$\mathrm{H}, 0,1.769782493,-2.4691134411,0.9251662546$

$\mathrm{H}, 0,-0.0001387582,2.0805885798,-1.915961493$

$\mathrm{H}, 0,-1.0129701122,-4.3993620653,-0.9076475466$

$\mathrm{H}, 0,-1.8096416894,-4.7040009833,0.6396646999$

$\mathrm{H}, 0,-1.6643097363,1.9976730027,-1.315540421$

$\mathrm{H}, 0,0.2360993728,-4.095502495,1.8709458826$

$\mathrm{H}, 0,0.8805937406,-5.0346420322,0.5190900101$

$\mathrm{H}, 0,-2.6653448627,1.7137049957,0.5929679743$

C, $0,-2.8825770649,0.6108718444,2.4481709452$

$\mathrm{H}, 0,-2.8525117676,-0.0211752261,0.3360257272$

$\mathrm{H}, 0,0.3880909077,1.3228754588,2.5786796804$

$\mathrm{H}, 0,-0.1952789006,-0.320934266,2.8693172371$

$\mathrm{C}, 0,-1.7067909356,1.2031375705,3.2365681442$

$\mathrm{H}, 0,-3.8263860842,1.1332618065,2.6182690529$

$\mathrm{H}, 0,0.590482597,-1.2152797392,-2.5871868297$

$\mathrm{H}, 0,-3.026411041,-0.4403947358,2.7182931707$

C, $0,-1.3962067131,1.3179204242,-3.3865588428$

$\mathrm{H}, 0,-1.7314405831,2.2959864459,3.1850019958$

$\mathrm{H}, 0,-1.7022120863,0.9178454819,4.290161062$

C, $0,-0.5148229299,0.1628561965,-3.8801947711$

$\mathrm{H}, 0,-1.1348068962,-1.5818748976,-2.7104998056$

$\mathrm{H}, 0,-0.9240532067,-0.3517601693,-4.7516557203$

$\mathrm{H}, 0,0.4742212145,0.5423515976,-4.1519271413$

$\mathrm{H}, 0,-1.2800858444,2.2304263314,-3.9745945878$

$\mathrm{H}, 0,-2.4527145108,1.0324239099,-3.405578819$

C, $0,4.0606422711,3.4228973401,0.4278844794$

C, $0,3.758463013,1.0311855301,0.2169408941$

C, $0,1.8336520406,2.4511260579,0.3816249792$

C, $0,2.677847795,3.5467914138,0.4661513871$

C, $0,4.5889125903,2.1383475847,0.3024958976$

$\mathrm{Cl}, 0,4.4972022584,-0.558759039,0.0622515947$

$\mathrm{H}, 0,0.7627620385,2.5982638057,0.4207929984$

$\mathrm{Cl}, 0,1.9615700537,5.1570955425,0.62803584$

$\mathrm{H}, 0,5.6620412454,1.9968267199,0.2704500225$

$\mathrm{H}, 0,4.7043792603,4.2890466417,0.4940744223$

2-NO $-4-\mathrm{Cl}_{2}-\mathrm{C}_{6} \mathrm{H}_{3} \mathbf{P}_{\mathbf{1}}$ (pyrr)

c1256.log.acs
$2,5-\mathrm{Cl}_{2} \mathrm{C}_{6} \mathrm{H}_{3}-\mathrm{P}_{1}$ (pyrr)H+
2-NO ${ }_{2}-\mathbf{4 - C l}-\mathrm{C}_{6} \mathrm{H}_{3} \mathbf{P}_{\mathbf{1}}$ (pyrr)H+

cl256+.log.acs 


\section{Brutoformula: C18H27C11N5O2P1 \\ 0,1}

N,-0.0556677951,-1.9178866934,0.0840032114 P, $-0.0849627854,-0.2425375777,0.1187359531$ N,-0.7365434686,0.1768318758,-1.3743964222 N, 1.3809055574,0.1674729977,0.4933662314 $\mathrm{N},-1.2494127911,0.221388745,1.2138072185$ C, 2.3608792543,1.0905678808,0.447551791 C,-1.2864420173,-2.7295888266,0.1254140858 C, $1.1517401168,-2.7619429237,0.0161615939$ C, $-0.9073519436,0.3712450745,2.6471982263$ C,-2.6969304037,0.3881613362,0.9857732571 C, $-0.3776330434,-0.6036365825,-2.5881591844$ C,-1.1212584738,1.5457483426,-1.7767561692 H,-2.0051127977,-2.4191110621,-0.641122525 $\mathrm{H},-1.7808155995,-2.6317765981,1.0998029665$ C,-0.7930904633,-4.16715033,-0.1044046691 H, $1.5580246727,-2.7994976899,-1.0042955387$ C,0.6404031913,-4.1427083827,0.4438595751 H, 1.9275565351,-2.3668412431,0.6702615783 H,- $0.2469018473,2.1944645343,-1.9144807675$ $\mathrm{H},-0.7783102847,-4.3910525883,-1.1756058582$ $\mathrm{H},-1.4334260374,-4.9062185591,0.3812093515$ H,-1.7631107529,2.0100000671,-1.0315373428 H,0.6293589857,-4.2141298038,1.5359794493 H,1.2615741402,-4.9542109004,0.059301181 $\mathrm{H},-2.9207177347,1.3940582469,0.6089764376$ C,-3.3007805717,0.2098905488,2.3832766597 H,-3.0763336683,-0.3333403749, 0.2604338359 $\mathrm{H},-0.1112864611,1.1044819849,2.7760023783$ $\mathrm{H},-0.5663242073,-0.5852720226,3.0637337255$ C,-2.226124528,0.8193870507,3.2942620415 $\mathrm{H},-4.2744511131,0.695552254,2.4773297144$ $\mathrm{H}, 0.7024694646,-0.7658868275,-2.6684460877$ H,-3.4302142346,-0.8544046156,2.6054991697 C,-1.8027991923,1.3079627747,-3.1233375173 H,-2.2955446709, 1.9106340885,3.274426419 $\mathrm{H},-2.3056155826,0.4958848519,4.3338876547$ C,-0.8949873018,0.2492909407,-3.770926372 $\mathrm{H},-0.8577761956,-1.5844083908,-2.5504792611$ $\mathrm{H},-1.4124346372,-0.359576775,-4.5145652863$ $\mathrm{H},-0.0564350272,0.7378870339,-4.2738421265$ $\mathrm{H},-1.8834315983,2.2203038397,-3.7175441439$ H,-2.8105495925,0.9122386507,-2.9655008025 C,4.6750782405,2.7732485785,0.2540705925 C,3.6991753116,0.5968652474,0.5387996527 C, $2.283591312,2.5090549832,0.289552474$ C,3.4197386612,3.3252758843,0.1639298117 C,4.8185918475,1.3932490136,0.4522101178 H,3.8074855767,-0.4719216755,0.6733721245 $\mathrm{N}, 1.0168846206,3.2208557226,0.2524187164$ $\mathrm{H}, 3.2804847727,4.3867185862,0.0161562868$ H,5.8073459175,0.9576770819,0.5304288272 $\mathrm{Cl}, 6.1039764691,3.7940011389,0.1348789466$ $\mathrm{O}, 0.9473942845,4.2636904015,-0.4008537548$ $\mathrm{O}, 0.0701178894,2.7590106855,0.8901825735$

$\mathrm{HF}=-1928.1929118 \mathrm{NImag}=0$

\section{Brutoformula: C18H28C11N5O2P1(1+)}

1,1

$\mathrm{N},-0.3970588164,-1.9144364109,0.1939007326$ $\mathrm{P},-0.2749470859,-0.2781984356,0.0509819018$ N,-0.8002085039,0.129487168,-1.4518100795 $\mathrm{N}, 1.3520491562,-0.0340540073,0.3811948629$ $\mathrm{N},-1.1202500912,0.6592806682,1.1174296718$ C,2.1201188268,1.1354778186,0.2834147932 C,-1.7047150005,-2.6012923799,-0.0111266801 C, $0.6419859169,-2.881291815,0.6726296845$ C, $-0.6791664457,0.6927040774,2.5536803807$ C,-2.601969828,0.8763752364,1.0400246997 C, $-0.3676727168,-0.6923222027,-2.6304479338$ C,-1.2865187273,1.4699748543,-1.9043272228 H,-2.2011172177,-2.2328539977,-0.9104258889 $\mathrm{H},-2.3669128463,-2.4274262006,0.8446836548$ C,-1.3133000271,-4.0774651013,-0.1013999359 H, $1.416358715,-3.0054421673,-0.089956029$ C,-0.142557865,-4.1839958294,0.883643282 H,1.1149945248,-2.5381472288, 1.5941689054 H,-0.5309376494,2.2378806436,-1.7197454589 $\mathrm{H},-0.984887939,-4.3179147935,-1.1163519183$ $\mathrm{H},-2.145316682,-4.736475064,0.1490368242$ H,-2.2009844887, 1.7483243387,-1.3846697683 $\mathrm{H},-0.5163347942,-4.2349186147,1.9098146139$ $\mathrm{H}, 0.483996078,-5.0598357958,0.7134709355$ $\mathrm{H},-2.7974337095,1.8951948654,0.6944492239$ C,-3.0834767547,0.7007607552,2.4819436365 $\mathrm{H},-3.0706219157,0.1834639477,0.3404496048$ H,0.2028308827,1.3234204772,2.6679599193 H,-0.428954398,-0.3149790941,2.9004347176 C,-1.9020968574,1.2329297525,3.3011877669 H,-4.0145339901,1.2376407974,2.6677004011 $\mathrm{H}, 0.6237376698,-1.1204115213,-2.4723313508$ H,-3.2556212926,-0.3571760376,2.7018654097 C,-1.5064548562,1.2774194493,-3.4075221196 H,-1.8974409741,2.3265153233,3.2939025193 $\mathrm{H},-1.9142196718,0.9063889486,4.3414269672$ C,- $0.3997983881,0.2935242384,-3.8026001286$ H,-1.0738097115,-1.5131029006,-2.782432932 H, $-0.589773431,-0.211761539,-4.7500812632$ $\mathrm{H}, 0.5587579431,0.8131847533,-3.8858535253$ $\mathrm{H},-1.4549093766,2.2240580865,-3.9465383878$ H,-2.4916841088,0.8395656187,-3.5912255259 C,3.6852444435,3.4884457501,0.1206476562 C,3.5295128487,1.0879731711,0.1402341322 C, $1.5387403899,2.4121752993,0.3429389834$ C, $2.2986621108,3.5679231749,0.2613661216$ C,4.2995813476,2.2517621331,0.0627831975 $\mathrm{N}, 4.2767233085,-0.1775269332,0.0582977573$ $\mathrm{H}, 0.4680715792,2.4942952569,0.4700243891$ $\mathrm{H}, 1.8171388189,4.5362331134,0.3159091463$ H,5.3711405244,2.1613139774,-0.0441913878 $\mathrm{Cl}, 4.637204455,4.9444199152,0.0244638118$ H, 1.9554013974,-0.860370353,0.3719225764 O, $5.4707078971,-0.1339743254,-0.1548728397$ O,3.6527273238,-1.2427408597,0.2099274948

$H F=-1928.6109188$ NImag $=0$ 


\section{$\mathbf{P h P}_{\mathbf{1}}$ (tmg)}

tmgph.log.acs

tmgph.log

Charge $=0$ Multiplicity $=1$

$\mathrm{N}, 0,-0.4058673018,-0.7042641569,-1.2008891738$ $\mathrm{P}, 0,-0.1027790908,-0.3316878155,0.3979906325$ $\mathrm{N}, 0,1.5436106661,-0.4233183524,0.6418007066$ $\mathrm{N}, 0,-0.8096829549,-1.3234145708,1.4243279791$ $\mathrm{N}, 0,-0.6011634515,1.2405974847,0.6196534713$ C, $0,-0.0154487174,-1.6264560281,-2.0176270521$ C, $0,-0.8949059245,2.2695134946,-0.1018870209$ C, $0,2.3564266405,0.1738786977,1.451375251$ C, $0,-2.1507083314,-1.6031274495,1.5678653277$ $\mathrm{N}, 0,0.8417619215,-2.6853766202,-1.7814488495$ $\mathrm{N}, 0,-0.478987405,-1.5755739345,-3.3425846049$ $\mathrm{N}, 0,-0.2997447713,2.6447783606,-1.2948327857$ $\mathrm{N}, 0,-1.8844097622,3.1396752421,0.3419978175$ $\mathrm{N}, 0,2.0642297758,0.8285128844,2.6298793577$ $\mathrm{N}, 0,3.7191034725,0.1891599318,1.1228550914$ C, $0,-4.8801424899,-2.2896171289,2.0417588986$ C, $0,-3.1855478896,-1.1967946765,0.6880791499$ C, $0,-2.5451479318,-2.3793101566,2.6862686784$ $\mathrm{C}, 0,-3.8736173677,-2.7111310561,2.9155477607$ C, $0,-4.5161548698,-1.5331309018,0.9284390195$ H,0,-2.9298432629,-0.6303318052,-0.1998677882 $\mathrm{H}, 0,-1.7694121459,-2.7097319026,3.3687453821$ H,0,-4.1293340552,-3.3060117245,3.7874092683 $\mathrm{H}, 0,-5.2788790679,-1.2018071069,0.2292082344$ $\mathrm{H}, 0,-5.9170137444,-2.5497000198,2.2218081337$ C, $0,-1.1981163555,-0.3795140643,-3.7546573119$ C, $0,-1.03373547,-2.7906709052,-3.943777938$ C, $0,1.7999216769,-3.1381785131,-2.7799880885$ C, $0,0.9775256343,-3.3360324736,-0.4839606374$ C, $0,-1.019905555,3.3266777727,-2.3608785928$ C, $0,1.0596903343,2.2476776619,-1.6174445516$ C, $0,-2.7662490869,2.6814077872,1.4110136306$ C, $0,-1.6694295274,4.5845391322,0.3411204219$ C, $0,4.0961877093,-0.2220637617,-0.2220383082$ C, $0,4.7069794154,-0.1943830911,2.1312248605$ C, $0,2.7762704318,2.0287258651,3.0492880651$ C, $0,0.8887502654,0.5142064984,3.4354174306$ $\mathrm{H}, 0,-0.6795688695,0.5055652701,-3.3986444582$ $\mathrm{H}, 0,-2.2222936903,-0.3536097406,-3.3587493053$ $\mathrm{H}, 0,-1.2414539833,-0.3590422745,-4.8472681171$ $\mathrm{H}, 0,-0.8342533511,-2.8051583138,-5.0195702554$ $\mathrm{H}, 0,-2.1216832269,-2.8347625542,-3.7942004291$ $\mathrm{H}, 0,-0.5978213732,-3.6793609201,-3.4963654488$ $\mathrm{H}, 0,-0.6321785135,4.3380833496,-2.5402769171$ $\mathrm{H}, 0,-2.0773555301,3.3936693031,-2.1144243392$ $\mathrm{H}, 0,-0.918021289,2.7623371305,-3.2945151144$ $\mathrm{H}, 0,1.5636609036,1.8986538807,-0.7202622645$ $\mathrm{H}, 0,1.6034577278,3.1130911567,-2.014785746$ $\mathrm{H}, 0,1.092111641,1.4443587243,-2.3608553166$ $\mathrm{H}, 0,-0.8290523627,4.8513543129,-0.294983226$ $\mathrm{H}, 0,-1.449557458,4.9349698249,1.3587569237$ $\mathrm{H}, 0,-2.5601892283,5.1136792746,-0.0132649595$ $\mathrm{H}, 0,-2.3103135368,2.8154232834,2.4004926308$ $\mathrm{H}, 0,-2.994518994,1.6262937589,1.2849177733$ $\mathrm{H}, 0,-3.6917607556,3.2627893685,1.3707304636$

\section{$\mathbf{P h P}_{\mathbf{1}}$ (tmg)H+}

tmgph + .log.acs

tmgph+.chk

Charge $=0$ Multiplicity $=1$

$\mathrm{N}, 0,-0.5933984429,-0.0180077953,-1.2327887613$ $\mathrm{P}, 0,-0.2184065909,-0.2586081411,0.3403362631$ $\mathrm{N}, 0,-0.3916067669,-1.8269316643,0.754402096$ $\mathrm{N}, 0,-1.3368064234,0.5050296135,1.3596345094$ $\mathrm{N}, 0,1.2418929094,0.3760727047,0.6675691179$ C, $0,-1.7182831119,0.30341731,-1.8398572304$ C, $0,2.2880352994,0.7484291005,-0.03413279$ C, $0,0.3920094337,-2.6978720601,1.3547050354$ C, $0,-1.7676692523,1.8425876743,1.4081393313$ $\mathrm{N}, 0,-2.9889421883,0.0406867336,-1.3963341151$ $\mathrm{N}, 0,-1.6330675977,0.9669090699,-3.0367171353$ $\mathrm{N}, 0,2.7210408361,0.1710094242,-1.2011548422$ $\mathrm{N}, 0,3.0586971439,1.7721777416,0.4511825666$ $\mathrm{N}, 0,1.2240866395,-2.4300595733,2.40978574$ $\mathrm{N}, 0,0.3592296634,-3.9975862733,0.9238261583$ C, $0,-2.7002191032,4.4939702399,1.5824971105$ C, $0,-1.2480317508,2.8374029313,0.5688296819$ C, $0,-2.7599953659,2.1960005383,2.336112606$ C, $0,-3.2185587697,3.5056407267,2.4192718741$ C, $0,-1.7146824109,4.1479935936,0.6621492857$ $\mathrm{H}, 0,-0.4829285996,2.592740971,-0.1560984878$ $\mathrm{H}, 0,-3.1668361729,1.4390827527,2.9999136786$ $\mathrm{H}, 0,-3.9836565774,3.7543016753,3.145876973$ $\mathrm{H}, 0,-1.2986904143,4.9041638579,0.0054526578$ $\mathrm{H}, 0,-3.0567012591,5.5143244263,1.6503370173$ C, $0,-0.3918323927,1.6227517795,-3.4260219329$ C, $0,-2.5545137729,0.7093661393,-4.144770968$ C, $0,-3.3275427562,-1.1490138516,-0.6247809457$ C, $0,-4.1018610346,0.9666884262,-1.6081078303$ C, $0,3.3271150957,0.943827808,-2.2849741666$ C, $0,2.378355165,-1.2019815425,-1.551805178$ C, $0,2.5400694604,2.6325523489,1.5131321188$ C, $0,4.5182366121,1.7845071002,0.3267067482$ C, $0,-0.241836769,-4.3161774447,-0.3686906608$ C, $0,0.4553532684,-5.1349475915,1.8427295191$ C, $0,2.5197704608,-3.0867948356,2.5833048869$ C, $0,0.9656112797,-1.333817313,3.3404391283$ $\mathrm{H}, 0,0.2600136682,0.9472222346,-3.9929885748$ $\mathrm{H}, 0,0.1424659729,1.9613663823,-2.5430568275$ $\mathrm{H}, 0,-0.6302499633,2.4832448514,-4.0560084711$ $\mathrm{H}, 0,-3.2648638103,-0.0689213511,-3.8779020519$ $\mathrm{H}, 0,-1.9828219136,0.364832501,-5.0123438267$ $\mathrm{H}, 0,-3.1038566082,1.610616466,-4.4329033715$ $\mathrm{H}, 0,4.3614153551,0.6398643264,-2.4760504809$ $\mathrm{H}, 0,3.3080772005,2.0060333455,-2.0523644216$ $\mathrm{H}, 0,2.7516918741,0.7817768581,-3.2016959185$ $\mathrm{H}, 0,2.1846413542,-1.7788051266,-0.6496413369$ H,0,3.2317287026,-1.6515095878,-2.067055766 $\mathrm{H}, 0,1.4979368865,-1.2484423344,-2.198238202$ $\mathrm{H}, 0,4.8686134561,0.8846583155,-0.1728137728$ $\mathrm{H}, 0,4.9612447998,1.8143750015,1.3271027924$ $\mathrm{H}, 0,4.8702732864,2.6602306752,-0.2265110127$ $\mathrm{H}, 0,2.7700722279,2.2262798241,2.5048519484$ $\mathrm{H}, 0,1.4629847489,2.7368985892,1.4245101999$ H,0,3.007133973,3.6160013274,1.4241592213 
$\mathrm{H}, 0,3.2607434061,1.8924158709,4.0245448004$ $\mathrm{H}, 0,2.0636565512,2.8570810245,3.144451516$ $\mathrm{H}, 0,3.5320491386,2.3013457078,2.3161687346$ $\mathrm{H}, 0,1.195743238,0.4331952823,4.4848721325$ $\mathrm{H}, 0,0.4403240174,-0.4227721177,3.1103761845$ $\mathrm{H}, 0,0.1281288593,1.2965297893,3.3465448042$ $\mathrm{H}, 0,4.9653504009,-1.2584808558,2.0340496361$ $\mathrm{H}, 0,4.3188957179,-0.0328855063,3.1333330231$ $\mathrm{H}, 0,5.6235544487,0.3913929453,2.0108115631$ $\mathrm{H}, 0,5.1016091137,0.154327602,-0.43129779$ $\mathrm{H}, 0,3.3993667498,0.1877660483,-0.9489043636$ H,0,4.1001509144,-1.3146641137,-0.3356759938 H,0,1.7430191094,-2.5184950129,-3.6720390211 $\mathrm{H}, 0,1.6293798036,-4.1848266176,-3.0647619918$ $\mathrm{H}, 0,2.8142284754,-3.0691237966,-2.3670364587$ $\mathrm{H}, 0,0.8936341421,-4.4208203996,-0.6248362103$ $\mathrm{H}, 0,0.2002106983,-3.0064765047,0.2023653463$ $\mathrm{H}, 0,1.946961328,-3.1119648919,-0.0282457272$

$\mathrm{HF}=-1714.1506 \mathrm{NImag}=0$

\section{$\mathbf{E t P}_{2}$ (dma)}

etp2.log.acs

\section{Brutoformula: C12H35N7P2}

0,1

N,1.8755376066,-1.0123164033,-1.0541541228

$\mathrm{P}, 1.4031799411,-0.3880803635,0.4389627951$

N,1.2902511943,-1.7776826284,1.3914328059

$\mathrm{N}, 0.1131329472,0.4894829234,0.6164581724$

$\mathrm{N}, 2.7337738979,0.5791443955,0.8406667837$

$\mathrm{P},-1.2518684474,0.624238042,-0.2938498566$

$\mathrm{N},-1.86846121,-0.5704412408,-1.1061223051$

N,-0.8728080226,1.7950753086,-1.4844897372

$\mathrm{N},-2.3378377319,1.2528964204,0.8737887384$

C, $2.2638848825,-0.0570444335,-2.1035520027$

C, $1.1562006208,-2.1699174393,-1.6135284224$

C, $2.5751180528,1.7064166856,1.7572804196$

C,4.1034494716,0.0742487682,0.7851783851

C, $0.3158104914,-1.8777224581,2.4765206124$

C, $2.3783939766,-2.746396615,1.4956443701$

C,-0.3209318145,3.0704374843,-1.0482648091

C,-1.6959480258,1.9069649252,-2.686490091

C,-3.7045795455,1.5345113544,0.4497762523

C,-1.9421441265,1.9809031023,2.0706534348

C,-2.4888673949,-1.7242919831,-0.4801030071

H,4.4412110594,-0.3252229332,1.7519798408

$\mathrm{H}, 4.7775605821,0.8925689911,0.5089658967$

H,4.1944322051,-0.7057051035,0.0312002344

H,2.8367930636, 1.4354203682,2.790407112

$\mathrm{H}, 1.545864749,2.055319243,1.7354332474$

H,3.2340975908,2.5240023629,1.4445577774

$\mathrm{H}, 0.786473617,-1.7157404541,3.4564363492$

H,- $0.1336323747,-2.8770056643,2.4743858235$

H,-0.4713688914,-1.1411029946,2.338473517

$\mathrm{H}, 0.8919216852,-2.8696685691,-0.8235196317$

$\mathrm{H}, 1.8169158769,-2.6774324971,-2.3234918168$

$\mathrm{H}, 0.2363728707,-1.8592157055,-2.1202901384$

H,2.917535227,-0.5661694944,-2.8185900578
$\mathrm{H}, 0,2.5538580732,-3.689008018,3.4970014678$ $\mathrm{H}, 0,3.2987367035,-2.3208665278,2.6529025426$ $\mathrm{H}, 0,2.7409528057,-3.7251140123,1.7309818216$ $\mathrm{H}, 0,1.2478388174,-1.6609288624,4.3447200462$ $\mathrm{H}, 0,-0.0948130913,-1.092138749,3.3468517521$ $\mathrm{H}, 0,1.5253451948,-0.4335136259,3.0766775913$ $\mathrm{H}, 0,-0.4562535088,-5.7352305909,1.7638496595$ $\mathrm{H}, 0,0.5458143684,-4.7907221948,2.8698746693$ $\mathrm{H}, 0,1.3086280227,-5.7763271423,1.603625631$ $\mathrm{H}, 0,0.2410670664,-5.2109001419,-0.7683494595$ $\mathrm{H}, 0,-0.1012632135,-3.4900815037,-1.0604051717$ H,0,-1.3159919306,-4.5123410939,-0.2732165494 $\mathrm{H}, 0,-2.4570036659,-1.785919354,-0.4956546995$ H,0,-4.1099630032,-1.7091999628,-1.1495331134 $\mathrm{H}, 0,-3.7132937068,-0.8764193585,0.3627892323$ $\mathrm{H}, 0,-4.8605689235,0.5525168253,-2.2808068711$ $\mathrm{H}, 0,-3.7392732368,1.9083592658,-2.0125034688$ $\mathrm{H}, 0,-4.5746002661,1.1699118405,-0.6428469985$ H,0,-1.8291534561,-0.1276356928,1.9728927294

\section{$\mathrm{EtP}_{\mathbf{2}}$ (dma)H+}

etp2+.log.acs

Brutoformula: C12H36N7P2(1+)

1,1

N,1.7716543076,-1.179968664,-1.1230383841

$\mathrm{P}, 1.3357202893,-0.5027959776,0.3390139182$

$\mathrm{N}, 1.2837081711,-1.8317061805,1.361064491$

$\mathrm{N},-0.0430753285,0.26285558,0.5073520522$

N,2.5687718926,0.5961923723,0.6306723511

P,-1.3347049921,0.8760891496,-0.1697456989

H,-2.9429022597,0.0618061744,-1.5675724118

N,-1.2021529178,2.0552801903,-1.3496844316

$\mathrm{N},-2.202887896,1.6098894472,1.0481361788$

C, $2.3683201443,-0.3902669645,-2.2084740621$

C, $1.1574691212,-2.4246115502,-1.6051066771$

C, $2.3487447215,1.8176559661,1.4136271522$

C,3.9740603645,0.1648805678,0.6415283575

C, $0.4315392264,-1.8541866531,2.5551925252$

C, $2.4155515571,-2.7645594882,1.4572988199$

C,-0.8565127459,3.4337867486,-0.9764927203

C, $-0.8329662782,1.739714543,-2.7374157729$

C,-3.5473740316,2.1282090795,0.7740845256

C, $-1.8576125047,1.5609548645,2.4697525338$

C,-2.5335595363,-1.5993764066,-0.3681031274

$\mathrm{H}, 4.316694468,-0.0743932742,1.6550241332$

$\mathrm{H}, 4.5990548377,0.9740115658,0.2534818276$

H,4.1205363329,-0.7068605166,0.0067466908

H,2.6009138474, 1.6722544552,2.4713232453

$\mathrm{H}, 1.3123197285,2.1341217183,1.3398094816$

H,2.987004871,2.6123996368,1.0172515709

H,0.9756556669,-1.5272690224,3.4499206732

$\mathrm{H}, 0.0850287876,-2.8778329427,2.7233767204$

$\mathrm{H},-0.4332662533,-1.2128750149,2.4127866158$

$\mathrm{H}, 0.7361771187,-2.9928571248,-0.7793372457$

$\mathrm{H}, 1.9190436495,-3.0375953197,-2.0957314727$

$\mathrm{H}, 0.3643643961,-2.2147685864,-2.3315647828$

H,3.1992673316,-0.9476508669,-2.6511978165 
$\mathrm{H}, 2.8069063865,0.7815514557,-1.6711832562$ H,1.3870654725,0.3306112934,-2.6344512502 H,2.9840916936,-2.5889145228,2.3989066225 $\mathrm{H}, 3.029018847,-2.6939548143,0.6241147997$ H,1.962859213,-3.7594089298, 1.5440370096 $\mathrm{H},-1.0816637019,2.2950362785,-3.5081188293$ $\mathrm{H},-2.5464740878,2.5944664229,-2.5525920605$ H,-2.0729245195,0.9223298553,-2.9532042239 H, $0.2733951602,3.5094177967,-1.8579074567$ $\mathrm{H}, 0.3341554538,2.9248919222,-0.1898226981$ H,-1.0997795681,3.8008664616,-0.775372996 $\mathrm{H},-4.4058812272,1.2736287965,1.2517061721$ H,-3.9529167992,0.939251402,-0.4287492762 $\mathrm{H},-3.8560528199,2.5980573547,0.2070385276$ H,-2.6272895283,1.7387026826,2.8933897222 H,-1.9699892552,3.0730231091,1.9273927148 $\mathrm{H},-0.9353963611,1.6904305427,2.3615886379$ C, $-3.3809816371,-2.4623349568,-1.4816220263$ $\mathrm{H},-3.0984806565,-1.4536993782,0.398684985$ $\mathrm{H},-1.7344458478,-2.4394481331,-0.1065358883$ $\mathrm{H},-2.7987941768,-2.7741955009,-2.3536087888$ $\mathrm{H},-4.1831349139,-1.8084694729,-1.8361333014$ H,-3.8327511488,-3.3523130584,-1.0293137119

$\mathrm{HF}=-1544.6158879 \mathrm{NImag}=0$

\section{2-Cl- $\mathrm{C}_{6} \mathbf{H}_{4} \mathbf{P}_{2}$ (dma)}

c2lp2.log.acs

\section{Brutoformula: C16H34C11N7P2}

0,1

N,-0.8111784023,0.8305084882,-2.6206119901 $\mathrm{P}, 0.5863143984,0.95698642,-1.6757048987$ $\mathrm{N}, 1.8003774852,-0.0942667959,-2.1758141856$ $\mathrm{N}, 0.288573298,0.8594923736,-0.1508133644$ $\mathrm{N}, 1.1787019788,2.475170191,-2.139362593$ C,-0.8726113107,1.2659614552,-4.0167467375 C, $-1.7106070381,-0.2990276768,-2.345966332$ C, $2.5593119989,2.8037689744,-1.7626367339$ C, $0.2827523077,3.6188880005,-1.9142406657$ C, $2.1264619742,-1.3212680702,-1.4455301817$ C, $2.2631652998,-0.0980810725,-3.5628330219$ P,-0.2294663194,0.0969655876,1.1731291103 $\mathrm{N}, 0.881058511,0.3308701088,2.443441076$ $\mathrm{N},-0.4924938079,-1.4349331654,0.8529212014$ $\mathrm{N},-1.5288918333,1.048763815,1.7356384365$ C,-2.1150009544,0.7656973155,3.0473356439 C,-2.5238485902,1.5216491114,0.7761508946 C, $1.9997376948,-0.6078991793,2.545803753$ C, $1.2723621909,1.6980666018,2.7901671208$ C, $-0.890215118,-2.5037751592,1.6141177896$ $\mathrm{H},-0.6397235672,0.4483505269,-4.7128762677$ H,-0.1821352723,2.0869366987,-4.198958539 H,-1.8883994458,1.6116472077,-4.2382010649 $\mathrm{H},-1.5393113783,-1.1204377495,-3.0535933575$ $\mathrm{H},-2.7505723673,0.0283039923,-2.4468637119$ H,-1.5595068321,-0.6921209076,-1.3413299992 $\mathrm{H}, 0.6208344962,4.459544733,-2.5270114285$ $\mathrm{H}, 0.2824143684,3.9312532318,-0.861602223$
H, 2.7455682395,0.558166975,-1.8339120099 $\mathrm{H}, 1.6335915542,-0.1933746368,-2.9964929903$ H,3.0749303962,-2.5168434998,2.29715701 $\mathrm{H}, 3.0003855991,-2.7656082424,0.5396343913$ H,2.0317153134,-3.776520371,1.61520252 H,0.2310607861,1.9329735759,-2.9170911538 $\mathrm{H},-1.4117719244,2.3721039103,-3.416473029$ H,-1.0470831082,0.6984544496,-2.9666798318 H,0.222491184,3.6129161971,-1.055497528 H,-1.1765183311,3.6465064241,0.0414638034 H,-1.3684980626,4.1249155901,-1.6511305899 $\mathrm{H},-4.3184694645,1.478902004,1.2042156348$ $\mathrm{H},-3.7192422767,2.2146108733,-0.2987591448$ $\mathrm{H},-3.6570950351,3.1260519696,1.2093983577$ H,-2.5358006786,0.8975153325,3.0189602066 $\mathrm{H},-1.9359470977,2.5638825522,2.9016314491$ $\mathrm{H},-0.8384934415,1.2067069006,2.5954646628$ C,-3.1250700334,-2.5542298422,-1.3991171806 H,-3.2389982457,-1.4633197088,0.461320907 H,-1.6172695078,-2.0169398859,0.0524475305 $\mathrm{H},-2.4219246705,-2.7414914987,-2.2137019444$ H,-4.0472488906,-2.1520366232,-1.8291883109 H,-3.3724990994,-3.5090497858,-0.9299660042 N,-2.1923992935,-0.3048201653,-0.9928660172

$\mathrm{HF}=-1545.0660752 \mathrm{NImag}=0$

\section{2-Cl- $\mathrm{C}_{6} \mathbf{H}_{4} \mathbf{P}_{2}$ (dma)H+}

c2 1 p $2+. \log$.acs

Brutoformula: C16H35C11N7P2(1+) 1,1

N,-0.6044988744,0.8170519224,-2.9906832324 $\mathrm{P}, 0.6202934229,0.8224186011,-1.8424092706$ N,2.0667735601,0.1277311942,-2.321954791 $\mathrm{N}, 0.0816769905,0.0186197977,-0.5903435543$ $\mathrm{N}, 0.9860413854,2.4438882537,-1.7009482265$ C, $-0.461938585,1.5446374403,-4.2610024585$ C, $-1.5167628262,-0.3297666603,-3.1055054034$ C, $2.2790549496,2.91731326,-1.1905849732$ C, $-0.0777461579,3.4497253655,-1.5666997236$ C, $2.442464632,-1.2393653579,-1.9441256158$ C, $2.7544630428,0.5925397873,-3.5352238067$ P,-0.217009894,-0.1915190927,0.9448460913 $\mathrm{N}, 0.9556669129,0.1467256043,2.0809820595$ H,-0.5357994077,-2.2684858974,0.1173278433 $\mathrm{N},-1.5148401039,0.7326115907,1.4353013635$ C,-1.9527997849,0.8116955266,2.8395273909 C,-2.6064621005,1.0221372774,0.4936304295 C,2.112227359,-0.7490663858,2.2478989609 C, $1.2696264862,1.5390083306,2.4370234176$ C,-1.0289091078,-2.6479985263,2.0583691391 H, $-0.0644002439,0.9023239779,-5.0555564229$ H, $0.1926552737,2.4056474203,-4.1444651386$ H,-1.4455548801,1.90404702,-4.575460012 H,-1.14436782,-1.0754340174,-3.8186627439 $\mathrm{H},-2.4875182447,0.0259037367,-3.461117587$ H,-1.6562578372,-0.8058266431,-2.1385182983 H, $0.2021236755,4.3493694125,-2.122018729$ H,-0.2256764306,3.7278049612,-0.5166399024 
H,- $0.7378723487,3.3707385183,-2.1992839108$ H,2.9010338743,3.6412002951,-2.3777462328 H,3.2165523637,1.9541420732,-1.9352187989 H,2.6363710692,3.0957084901,-0.7064694715 $\mathrm{H}, 3.3457312195,-0.2678853122,-3.5858990854$ $\mathrm{H}, 2.0638863613,0.860066601,-4.0414232715$ $\mathrm{H}, 1.7861438337,-0.8938073901,-4.1503215807$ H,3.2085700695,-1.3796144588,-1.2777841847 H,1.8141801022,-2.2043575981,-2.0153361899 H,1.6064706002,-1.3531294693,-0.4919172765 H,-3.0016752754,2.4232515028,1.1747912698 $\mathrm{H},-3.3107048278,0.7768734447,0.5863393363$ H,-2.0461290002,1.7759488445,-0.1677926429 $\mathrm{H},-1.3368801481,0.4915034718,3.7575876673$ H,-2.8603597941,-0.040386931,3.012257458 $\mathrm{H},-2.6101126655,1.6713944723,3.4146487488$ $\mathrm{H}, 2.8357752603,-0.3274609095,1.8874699611$ $\mathrm{H}, 1.6773776521,-1.6158429417,2.2913221655$ H,2.369196497,-0.6156221131,3.5762397945 H,2.0690747693,2.0756337804,2.1326373515 $\mathrm{H}, 1.6411427804,1.717983926,3.8212044245$ $\mathrm{H}, 0.4143412792,2.3638876472,2.7125828323$ C,-1.6998806189,-4.8401448589,3.0630794876 C,-1.3247930712,-3.69420727,0.9740486317 C, $-0.8750721429,-2.5663085553,3.0306982957$ C,-1.2706142564,-3.6973412867,3.7349561036 C,-1.7206349172,-4.8281352903,1.6695031263 $\mathrm{Cl},-1.3701244017,-3.7572706134,-0.7923094291$ H,- $0.5352262791,-1.6946222797,3.575738458$ H,-1.239512868,-3.6838291365,4.8195974475 H,-2.0446396553,-5.6997302158,1.1138973088 H,-2.0097192287,-5.7256514933,3.6048944733

$\mathrm{HF}=-2156.709217 \mathrm{NImag}=0$

\section{BEMP}

bemp.log.acs

Brutoformula: C13H31N4P1

0,1

$\mathrm{N}, 0.1022656588,-0.4366747278,-1.38228914$ $\mathrm{P}, 0.1473283022,0.0094051152,0.1033009761$ $\mathrm{N}, 1.6700417544,-0.0007844462,0.8858080047$ $\mathrm{N},-0.8896558245,-1.0769875776,0.9003805052$ N,-0.3830261944,1.5845020207,0.4828599028 C, $0.7587829747,0.0664909171,-2.5893537298$ C, $2.3432833524,-1.2966061191,0.9277837118$ H, $1.7815056096,0.5923113317,2.9298638549$ C, $0.1342142559,2.4313548105,1.5573190053$ C,-1.7434029315,1.9213352064,0.0706954559 C,-1.3816164536,-2.301696128,0.2470398938 C,-0.9764619024,-1.0351496096,2.3603120804 C, $1.6492175851,2.3466453166,1.6710592372$ $\mathrm{H},-0.3325214523,2.1875712857,2.527821721$ H,- $0.1564077322,3.4629554273,1.3279764033$ H, 2.1030026915,2.7162888183,0.7466360362 C, $2.1147185216,0.9215435838,1.9299473931$ H, 1.9867569493,2.9896410777,2.4899020569 H,3.2107775601,0.8929208159,1.9476741685 H,-1.7732530771,2.9516506699,-0.3003072576
$\mathrm{H},-1.0172609422,3.0749667227,-1.966062317$ H,2.6293365741,3.7526767541,-1.8040328826 H,3.0216555779,2.1240282709,-1.2245406618 $\mathrm{H}, 2.1863982268,3.2712743039,-0.1577228088$ H,3.8354499504,0.5457281264,-3.3757180444 H,2.4931761928,1.6243204075,-3.7633966065 H,2.5102538194,-0.0330729405,-4.4015019322 H,3.5216496199,-1.2781492547,-1.7700144678 H,2.1969731721,-1.9574625717,-2.7357847018 H, $1.9314778133,-1.5351680958,-1.0324195818$ $\mathrm{H},-3.0196766422,2.0065371029,0.7278226082$ H,-3.4118179215,0.2827383584,0.5724412836 $\mathrm{H},-2.2373404208,1.0374570436,-0.5285738218$ $\mathrm{H},-1.1240833879,0.6225821861,3.5163180181$ H,-2.7499637017,0.0917976903,3.0502826456 $\mathrm{H},-2.3336552128,1.8186796681,3.0313237096$ H,2.9464805332,-0.4510205825,1.6018558861 $\mathrm{H}, 1.8397029606,-1.7776790089,2.023834015$ H,2.4495263273,-0.6982519184,3.2862130177 H,2.1287448615,1.908298442,1.8655989039 $\mathrm{H}, 1.521453519,1.5898085234,3.4996722191$ $\mathrm{H}, 0.4168555363,2.1890038486,2.2524528374$ C, $-2.1787727742,-4.3078373926,4.0230855656$ C, $-1.8411358237,-3.7407323015,1.7106914608$ C,-0.797340748,-2.4188128163,3.4198157194 C, $-1.3749122731,-3.2320149725,4.3899455286$ C,-2.4050034913,-4.5668103658,2.6743626139 Cl,-2.1414610887,-4.0949812826,0.0094690423 H,-0.154099741,-1.6013412266,3.7159277419 H,-1.1815014207,-3.0295857821,5.4366025986 H,-3.0196308488,-5.4020459466,2.3639819832 $\mathrm{H},-2.6221674016,-4.9481046908,4.7753516364$ $\mathrm{N},-0.4418362363,-1.8605641988,1.0397319873$

$H F=-2157.1462013 \quad$ NImag $=0$

\section{BEMPH+}

bemp+2.log.acs

Brutoformula: C13H32N4P1(1+)

1,1

H, $0.1035503345,1.1387846212,-1.6983159001$ $\mathrm{P},-0.0179805039,0.0246747571,0.2451003793$ $\mathrm{N},-1.4928257323,0.127336992,0.9852353817$ $\mathrm{N}, 0.9136340042,1.3252290048,0.7135689569$ $\mathrm{N}, 0.5367089044,-1.4938509681,0.6252078637$ C,-0.6685327646,-0.6582502404,-2.4804364297 C, $-2.3111233624,1.3314796005,0.7814322186$ $\mathrm{H},-1.70208852,-0.4354796883,3.0218611784$ C,- $-0.0094045423,-2.3145811487,1.7255058714$ C, $1.9333405191,-1.8134958843,0.2878750296$ C, $0.8594024104,1.7928276744,2.121099978$ C, $2.0408533851,1.8784732731,-0.0828948338$ C, $-1.5243023005,-2.2235602688,1.8097595446$ $\mathrm{H}, 0.4499677051,-2.0304560023,2.6825867817$ $\mathrm{H}, 0.289746403,-3.346288269,1.5232573385$ $\mathrm{H},-1.969753032,-2.6283678849,0.8966023304$ C,-1.9959971887,-0.793915669,2.026322396 $\mathrm{H},-1.8714327387,-2.8401344975,2.6426632237$ H,-3.0870889832,-0.7549105645,1.9804080143 H,1.9966927968,-2.8641104163,-0.0036998019 
$\mathrm{H},-2.4602151971,1.830176884,0.8988612851$ H,-2.0645788812,1.2616838709,-0.7342965133 C, $0.3693602704,1.5293838795,-2.8950008348$ C, $0.2740833974,-0.8231903526,-3.7511637754$ C, $2.297093705,-0.0368015469,-2.5024293538$ $\mathrm{H},-0.8136712327,-0.7665521819,-3.8416633605$ $\mathrm{H}, 0.5419955822,-1.8663152432,-3.5636749521$ $\mathrm{H}, 0.7204815909,-0.5140258896,-4.7021604567$ $\mathrm{H}, 2.7633665602,0.2894361853,-3.4380855217$ $\mathrm{H}, 2.5983050317,-1.0714606034,-2.317026044$ $\mathrm{H}, 2.6876641193,0.582023075,-1.6910292876$ $\mathrm{H}, 0.824271345,1.8673209458,-3.8320262209$ $\mathrm{H}, 0.6964933172,2.1984772267,-2.0951385002$ $\mathrm{H},-0.7158102968,1.6216466024,-2.992074087$ H,-1.4299980134,-3.0834011116,1.0153987515 $\mathrm{H},-0.6530526676,-2.6186023768,-0.5001015655$ C,-2.7437654111,-2.1506035294,-0.4340593152 H,- $0.466317627,-1.9107228279,2.7908942978$ C,-2.4051989483,-0.9720380132,2.9109824096 H,- $0.4285368388,-0.160143097,2.7157734202$ H,-3.0535293432,-3.1052114648,-0.8729829605 H,-2.6748785138,-1.4151510498,-1.2373566054 $\mathrm{H},-3.5180505682,-1.8318243099,0.2682051885$ $\mathrm{H},-2.9793645869,-1.8614925459,2.6413469793$ $\mathrm{H},-2.9358911355,-0.0975917253,2.526702595$ H,-2.3831057452,-0.9115410914,4.0037837552 H,3.4247565599,-1.1548940074,0.8270592305 $\mathrm{H}, 2.0089164278,-1.9200713005,0.0986780444$ H,2.1536274493,-1.8352321858,1.8681531158

$\mathrm{HF}=-1074.6384516 \mathrm{NImag}=0$

\section{DBD}

dbd1.log.acs

\section{Brutoformula: C8H14N2}

0,1

C,-1.4769739184,2.1863904145,0.1546306042 $\mathrm{N},-1.3835568494,1.0895717098,-0.8035200091$ C,- $-0.6527087372,0.073233564,-0.5230840051$ $\mathrm{H},-0.9644741919,-0.6760450187,-2.4729090853$ H,-2.0001582915,1.1235001958,1.9605573351 $\mathrm{H},-2.4804616771,2.616165464,0.0778344175$ H,-0.7830963198,2.9823520732,-0.1495970996 C, $0.1175491344,0.9727320715,1.6220975902$ C, $-1.187111047,1.7584516376,1.5935626501$ $\mathrm{H}, 0.9665757318,1.6406628346,1.4029503199$ $\mathrm{N}, 0.0709391792,-0.1226435537,0.6537823666$ $\mathrm{H}, 0.2966449593,0.5416797905,2.6128642864$ C,1.2558567701,-0.9855911909,0.6858997499 H,-1.4403980747,-1.7795100656,-1.1899133358 H,2.1431990504,-0.4116911084,0.3666829946 C, $1.1194554371,-2.2284207078,-0.1866416775$ $\mathrm{H}, 1.426224581,-1.2712029855,1.7291118454$ H,2.0784915754,-2.7551204479,-0.2023850907 C, $0.674451462,-1.8332210818,-1.59418007$ $\mathrm{H}, 0.3821631366,-2.9087281556,0.254834238$ $\mathrm{H}, 1.4377582822,-1.1951967835,-2.0568028448$ C,-0.6560650687,-1.0813722681,-1.5094694168
H,2.6024414725,-1.6421337594,1.1380284197 H,2.2734076049,-1.2136917421,-0.5549554661 C,-2.1919547118,-0.7183493245,-2.2871291496 C,-0.0736376705,-2.0716717027,-2.5144296431 C, $-0.3377519766,0.0651576273,-3.7946597704$ H,-0.2817439399,-2.6251058602,-1.5985568599 $\mathrm{H}, 1.0068755004,-2.0392393695,-2.667541697$ $\mathrm{H},-0.5132766858,-2.6249470841,-3.3471454246$ H,- $0.7724741114,-0.4756908753,-4.6369635912$ $\mathrm{H}, 0.7420015725,0.1241402779,-3.9521868189$ $\mathrm{H},-0.751454905,1.0786819812,-3.8072491116$ H,-2.6491740167,-1.2791731493,-3.1055901983 $\mathrm{H},-2.6286906793,0.2834788462,-2.2829106393$ $\mathrm{H},-2.4590212664,-1.2151786421,-1.3522426239$ H, $-0.1500926374,1.6317767361,2.5006941392$ H,1.0081544944,2.8751810224,2.1035679975 C, $1.8777499277,1.1372635007,3.0555659176$ H, 2.9216006823,1.9181063639, 0.5633575335 C, $1.7523111502,3.2669950643,-0.6542914478$ H,2.2849128537,1.1856349398,-0.8877632631 $\mathrm{H}, 1.8009324616,1.5799688886,4.0518857421$ H,2.9039718391,1.2830900718,2.7119992081 $\mathrm{H}, 1.7011478851,0.06305862,3.1529969456$ H,1.5118151562,3.9876215634,0.1301922695 H,0.9183077869,3.2538777947,-1.3622494063 H,2.6323963217,3.6384866397,-1.1852777383 $\mathrm{H},-3.3122440032,1.0449953554,0.449558739$ $\mathrm{H},-1.8716022764,1.9776337221,0.0220586703$ H,-2.3988652584,1.9083866855,1.7076971098 N,-0.0194093654,0.1802413879,-1.3995993646

$\mathrm{HF}=-1075.0709345 \mathrm{NImag}=0$

\section{DBDH+}

$\mathrm{dbd}+. \log$.acs

Brutoformula: C8H15N2(1+)

1,1

C,-1.7485517381,2.1347061176,0.2376301224

$\mathrm{N},-1.5726254534,1.0025409207,-0.6831714046$ C,-0.6449461819,0.0549899239,-0.5495120451 C, $-0.402429542,2.4474061083,0.8795755397$ H,-2.4995762913,1.8781785014,0.9909768923 $\mathrm{H},-2.1230159552,2.9818496045,-0.3374165568$ H, $0.2710117165,2.8809510064,0.1353846016$ C, $0.2064425362,1.1729278087,1.4524433855$ $\mathrm{H},-0.5275704432,3.1809988753,1.6772322739$ $\mathrm{H}, 1.2423681967,1.3403218859,1.751428398$ $\mathrm{N}, 0.2126709432,0.0766855722,0.4554032147$ H,-0.3418154082,0.8277480996,2.3346917983 C, $1.2678467861,-0.9500378436,0.6587488893$ H,-1.4870422477,-1.7051003736,-1.3403190372 $\mathrm{H}, 2.2241590441,-0.4863685321,0.3944596906$ C, $1.0375537044,-2.2187892638,-0.1500069638$ H,1.29063348,-1.1661344859,1.7292000987 H, $1.9498034823,-2.8177262383,-0.1161964208$ C, $0.6586670563,-1.8652200591,-1.5869548654$ $\mathrm{H}, 0.244996282,-2.8184809911,0.3097825021$ H,1.4609215695,-1.2898957813,-2.0601393834 C,-0.6374176038,-1.0526653452,-1.5758783857 
$\mathrm{H}, 0.5688118108,-2.7142856936,-2.2338012961$ $\mathrm{H},-1.1192056834,2.6234985345,2.2600443481$

$H F=-422.8978604$ NImag $=0$

\section{DBN}

dbn.log.acs

Brutoformula: C7H12N2

0,1

C, $0.9464378744,2.1512749103,-0.2471679915$ $\mathrm{N}, 1.2374721543,0.9456405836,-1.0240045654$ C, $0.6376584773,-0.1129670502,-0.6308807922$ $\mathrm{H}, 1.6557242884,2.2225458127,0.5907037715$ C,-0.4917769768,2.185328694,0.2893212743 H,1.1308371956,3.0219352977,-0.8821638239 C, $-0.7835364351,0.9274780454,1.1147505383$ H,-1.1817861646,2.2314584408,-0.5597614566 H,-0.6633201205,3.0764446917,0.8994717542 $\mathrm{H},-0.3573504368,1.01726337,2.1226408919$ $\mathrm{N},-0.2263812609,-0.2380963605,0.4405396369$ $\mathrm{H},-1.865004409,0.7889547019,1.2336925087$ C, $0.7547482842,-1.4721699594,-1.2926561934$ H,- $0.8894227676,-1.8750510181,1.5998713771$ H, $1.7867260307,-1.6892465109,-1.5671546308$ $\mathrm{H}, 0.1644684524,-1.4622254824,-2.2151891221$ C, $0.1631604651,-2.443750837,-0.2538464353$ $\mathrm{H}, 0.9523469203,-2.813140778,0.4061158553$ $\mathrm{H},-0.3308043173,-3.307550282,-0.7009733838$ C,-0.8125969072,-1.5667189908,0.5521999514 H,-1.8276003461,-1.5874072776,0.1244908353

$\mathrm{HF}=-383.5733612 \mathrm{NImag}=0$

\section{DBU}

dbu.log.acs

\section{Brutoformula: C9H16N2}

0,1 C, $-0.9470146557,-0.1529434344,-0.2831100645$ $\mathrm{N},-1.5336516699,-1.2041161331,-0.7333257908$ C,-1.3492164941,-2.4955189362,-0.0829498522 C,-0.2220076925,-2.5312415589,0.9499216307 $\mathrm{H},-1.1816974039,-3.2399196294,-0.8686974245$ $\mathrm{H},-2.2983865984,-2.7735312345,0.3940806029$ $\mathrm{H}, 0.7541877946,-2.5566963168,0.4523515084$ $\mathrm{H},-0.2933113875,-3.4230693213,1.5802550698$ C,-0.3292221454,-1.2576359144,1.7858401924 $\mathrm{H}, 0.4618606345,-1.1976877361,2.535201655$ $\mathrm{H},-1.2855525001,-1.2466242361,2.3230469497$ $\mathrm{N},-0.2495390332,-0.0678686963,0.9267338688$ C, $0.9790814468,0.7169478844,1.1476580319$ C,-1.150618124,1.1308632564,-1.0634665004 H, $1.8411757494,0.0336180341,1.0663681797$ $\mathrm{H}, 0.9564841087,1.0632121743,2.1872583938$ C, $1.2774753216,1.9154015947,0.2415496755$ C, $0.0226218557,1.5385769983,-1.9707547127$ H,-1.3760340665,1.9386556311,-0.3614240618
$\mathrm{H}, 0.5147050485,-2.7639327629,-2.1883926564$ $\mathrm{H},-0.8436685744,-0.611745541,-2.5548743784$ $\mathrm{H},-2.1966451317,0.9227620835,-1.4730349416$

$\mathrm{HF}=-423.3116458$ NImag $=0$

\section{DBNH+}

dbn+.log.acs

Brutoformula: C7H13N2(1+)

1,1

C, $0.8409158419,2.1909309974,-0.175320706$

$\mathrm{N}, 1.1170512913,0.9184706442,-0.8701594888$

C, $0.5159975003,-0.2102246832,-0.5319338793$

$\mathrm{H}, 1.5771821175,2.3286329645,0.6218515557$

C,-0.5834327871,2.1631876287,0.380129797

H,0.9646490104,3.0007852297,-0.8941933905

C,- $0.8330950995,0.8924983879,1.1968848125$

$\mathrm{H},-1.2984687682,2.2159536837,-0.4452674431$

H,-0.7466229826,3.0386225494,1.0100565677

$\mathrm{H},-0.3102836577,0.9187801271,2.1577426695$

$\mathrm{N},-0.3712169071,-0.2816902418,0.4408607695$

$\mathrm{H},-1.8977345543,0.7643025405,1.4003584745$

C, $0.7113290728,-1.5441111383,-1.1954883435$

H,-0.9663158236,-1.8971246871,1.6675149156 $\mathrm{H}, 1.7646461081,-1.7588303903,-1.3823928193$ $\mathrm{H}, 0.2001915804,-1.528107416,-2.1642562507$ C,0.047452773,-2.519426797,-0.1959799573 $\mathrm{H}, 0.8025649617,-2.9422424148,0.4682187588$ H, $-0.4588567771,-3.3436677677,-0.695073037$ C,-0.9293342306,-1.6413338258,0.6077652447 H,-1.948669292,-1.6605163431,0.2105296608 H,1.8020506227,0.895110952,-1.611847913

$H F=-383.9841683$ NImag $=0$

\section{DBUH+}

$\mathrm{dbu}+. \log$.acs

Brutoformula: C9H17N2(1+)

1,1

C,-0.8904406428,-0.1197927618,-0.245381452

$\mathrm{N},-1.4383538083,-1.2709861928,-0.6291080578$ C,-1.2617746844,-2.547590166,0.0784754467 C, $0.0800238583,-2.5223868836,0.8017161287$ $\mathrm{H},-1.3013866805,-3.3496267664,-0.6589678868$ $\mathrm{H},-2.0901409742,-2.690238716,0.7789112626$ H,0.8926730613,-2.5721199117,0.0721570267 $\mathrm{H}, 0.1696213856,-3.3932119253,1.4526019566$ C, $0.2054617325,-1.2503333683,1.6341487996$ H,1.2270723209,-1.1296991624,1.9964197082 $\mathrm{H},-0.4561307051,-1.2761927526,2.5057826375$ $\mathrm{N},-0.1248829602,-0.0491036959,0.8330552816$ C,0.4705026695,1.2452733527,1.2406041198 C,-1.1627594945,1.1192273519,-1.0569306795 H,0.7769255803,1.1378340454,2.2809137034 $\mathrm{H},-0.316045593,2.000517816,1.2278202379$ C, $1.6540769221,1.6667443886,0.3516823637$ C, $0.0976270224,1.7499830397,-1.7324659114$ H,-1.638730791,1.842912723,-0.3897517205 
H,-2.0381702353,0.973697599,-1.6764874081 C, $1.383549848,1.5629476757,-1.249629939$ H,2.2385394814,2.3058217085,0.5927243434 $\mathrm{H}, 0.5512304079,2.7185386442,0.4005184004$ H,1.8695418226,0.5840190714,-1.3387980541 H, 2.0467395394,2.2734096282,-1.7522823482 $\mathrm{H},-0.1984244233,2.5321676397,-2.3759255932$ $\mathrm{H}, 0.0703584224,0.8589756064,-2.8266567542$

$H F=-462.2065258$ NImag $=0$

\section{TBD}

tbd.log.acs

\section{Brutoformula: C7H13N3}

0,1

C, $-0.4807593719,2.4619013696,-0.8574015546$

$\mathrm{N},-0.8122745214,1.1576524314,-1.4108195039$

C,-0.3921970895,0.0954244634,-0.8224870863

$\mathrm{H}, 0.2801567385,-3.3097625985,0.9600748573$

H,-0.7511089084,-3.2018119926,-1.3659697389 $\mathrm{H}, 1.324549769,-2.5212361925,-0.2347155846$ C, $-0.7742315873,-2.3566905851,-0.6735971831$ H,- $0.2841293182,1.4785410673,1.7455265285$ $\mathrm{N}, 0.3783653796,0.0370639876,0.339908861$ $\mathrm{N},-0.6385630276,-1.1283079571,-1.4450732247$ C, $0.3973158532,-1.1688953704,1.1659827282$ H,-1.2907727765,-0.9987088777,-2.2060482814 H,-1.7313719411,-2.4044370815,-0.1277401085 H,1.2993688354,-1.1349232849, 1.7852030349 C, $0.3828921913,-2.4331710842,0.3146365871$ $\mathrm{H},-0.4635326881,-1.1774371087,1.8570704963$ C, $0.724534877,2.441557118,0.0867568954$ H,-1.3586422995,2.8607734965,-0.3284485941 $\mathrm{H},-0.294769979,3.150119039,-1.6887984339$ $\mathrm{H}, 1.6452761621,2.2967425134,-0.4875233693$ C, $0.5662295658,1.288142909,1.0697092706$ $\mathrm{H}, 0.8161215317,3.3882553686,0.6278989923$ H, 1.4575426039,1.1792083703,1.6958544116

$\mathrm{HF}=-438.9452158 \mathrm{NImag}=0$

\section{MTBD}

mtbd1.log.acs

\section{Brutoformula: C8H15N3}

0,1 C, $2.2048673711,0.3741066404,1.4443409223$ $\mathrm{N}, 0.7552190752,0.3999882664,1.5495771892$ C, $0.0265146872,0.2297556754,0.5037623286$ H, $2.5718115681,-0.5939163124,1.8153544448$ $\mathrm{H}, 2.6108426051,1.1280308485,2.1275551859$ C,2.7258816393,0.6124543539,0.0265571437 H,2.1564526693,-1.3287993084,-0.7432929722 $\mathrm{H}, 3.7941303324,0.3857819919,-0.0448078344$ $\mathrm{H}, 2.5888023686,1.6624129078,-0.251634611$ H,-2.3890727904,-0.7524956263,-2.4029998023 C, $1.9355862741,-0.2647489219,-0.9337439723$
H,-1.9102834637,0.879587004,-1.8149866497 C, $1.4537316166,1.3414743346,-1.1364647556$ H,2.5718395689, 1.1832806714,0.6979658895 H, 1.7999538418,2.7417171009,0.4941192699 H, 1.6031426889,0.2673703394,-1.293473544 H,2.2434927936,1.8302476639,-1.7117649125 $\mathrm{H},-0.0153263565,2.8365029118,-1.7026744899$ $\mathrm{H}, 0.0948732322,1.4705289656,-2.788089604$ H,-2.0194107428,-1.2514035332,-1.4539980807

$$
H F=-462.6267263 \text { NImag=0 }
$$

\section{TBDH+}

tbd+.log.acs

\section{Brutoformula: C7H14N3(1+)}

1,1 C,- $-0.3352341807,2.5433263911,-0.9189930592$ $\mathrm{N},-0.6698888849,1.2076231073,-1.4357661082$ C, $-0.4570437353,0.0589710841,-0.7680098307$ $\mathrm{H}, 0.4479995862,-3.3031886711,0.8731367756$ H,- $0.5485088254,-3.1503417416,-1.4943093023$ H,1.3886930299,-2.3330922836,-0.2608228267 C,-0.7207012619,-2.4183800692,-0.7045669837 $\mathrm{H},-0.3231625006,1.6365145418,1.7498578205$ $\mathrm{N}, 0.1482181231,0.0637759144,0.4258439445$ $\mathrm{N},-0.8483165469,-1.0963600485,-1.3364055897$ C, $0.3007968895,-1.1830065183,1.2026536149$ $\mathrm{H},-1.3200871684,-1.0421953816,-2.2262316135$ H,-1.6573655009,-2.6763622355,-0.2003521385 H,1.1925270853,-1.0688173997,1.8200553956 C, $0.4401663075,-2.3873704013,0.2799646585$ $\mathrm{H},-0.5568356177,-1.2971606979,1.8752280919$ C,0.8149856276,2.4187692723,0.0704607585 H,-1.216893172,2.9851605194,-0.4441101911 H,-0.0586547202,3.1696035124,-1.7677239091 $\mathrm{H}, 1.7395431093,2.1734895809,-0.4588500983$ C, $0.4999125006,1.3336577632,1.092793588$ H,0.9680814622,3.3698359473,0.5829391329 H,1.3691835619,1.1355999421,1.7209626829 H,-1.1445131383,1.1503889362,-2.3238367449

$H F=-439.3615636$ NImag=0

\section{MTBDH+}

$\mathrm{mtbd}+. \log$.acs

Brutoformula: C8H16N3(1+)

1,1

C,-1.3926441502,-0.3798792291,-2.2328338076 $\mathrm{H},-2.2985720939,-0.7253030352,-0.3348154079$ C,- $-0.4257928735,-0.0641364565,0.0475316013$ $\mathrm{H},-1.8022562822,0.5721921248,-2.5852722739$ $\mathrm{H},-2.0303486728,-1.1803961311,-2.6090757761$ C, $0.0463970562,-0.5573080156,-2.6914509726$ C, $0.9381339453,0.4236589016,-1.9429727543$ $\mathrm{H}, 0.1226072149,-0.3750246908,-3.7646343058$ $\mathrm{H}, 0.376630292,-1.5823048354,-2.5029716535$ $\mathrm{H}, 0.7347962746,1.4547847187,-2.2551964749$ H, 1.9894521926,0.2183558945,-2.1492706273 
$\mathrm{H},-1.8789332311,-0.3941740313,2.5504238888$ $\mathrm{H},-3.249441013,0.1175877832,-0.1835052543$ $\mathrm{N}, 0.5055273112,0.0023100606,-0.7960585825$ $\mathrm{N},-1.3599204351,0.375172693,0.6284598352$ C, $-1.8830340682,0.5444529232,1.9777263002$ H,-2.3436404786,-1.389662952,-0.0330042062 $\mathrm{H}, 0.1072534441,-0.4386589231,-2.7834269514$ C, $-0.322811212,-0.6564954372,-1.8006553578$ H,- $0.2922733066,-1.7533097168,-1.6749569846$ H,-2.9104983836,0.9090981455, 1.9039066415 H,-1.2755974203,1.2651927363,2.5186131661 C,-2.2532631899,-0.319382494,-0.2920164998 C,-1.760424854,-0.1709335598,-1.7228338091 $\mathrm{H},-1.8148020393,0.8798045559,-2.0219299835$ H,2.2118327074,-0.0605285309,-1.9731143089

$\mathrm{HF}=-478.2595151 \quad \mathrm{NImag}=0$

\section{ITBD}

tbdipr.log.acs

Brutoformula: C10H19N3

0,1

C, $-0.5791424688,2.1654757416,-1.1775820036$ $\mathrm{N},-0.4359840098,0.8032887408,-1.6620226116$ C, $-0.0066897051,-0.1313101473,-0.8892342336$ $\mathrm{H}, 0.900382649,-3.1647827493,1.4049352808$ $\mathrm{H}, 0.6647816371,-3.4031528469,-1.0940995176$ H,2.0292307133,-2.1396123325,0.5009625339 C, $0.2048582061,-2.5747297343,-0.5506530009$ H, $-1.0720889602,1.309644831,1.4061137354$ $\mathrm{N}, 0.3462816019,0.0452677645,0.4617125188$ N,0.222143093,-1.402634576,-1.4251036914 C, $0.390426588,-1.0717353876,1.397478712$ C,-0.0994610052,-1.6873604057,-2.8461652847 $\mathrm{H},-0.8254766145,-2.8856405275,-0.3062722204$ $\mathrm{H}, 0.9996112266,-0.766623018,2.2544689842$ C, $0.9729930178,-2.3052253725,0.7324412831$ H, $-0.617686055,-1.2987481813,1.7874828499$ C,0.2119794796,2.4591522809,0.096737017 H,-1.6458263207,2.3715862149,-1.0051444334 H,-0.2714381182,2.8480477407,-1.9781756468 $\mathrm{H}, 1.2809455387,2.5252095393,-0.1301005231$ C,- $0.0190462443,1.3191482864,1.0771441605$ H,-0.0940905106,3.4114700256,0.5407152454 $\mathrm{H}, 0.596288304,1.4403853547,1.9740234622$ $\mathrm{H}, 0.2000275763,-2.7339205436,-2.9636139592$ C, $0.7579970196,-0.8850533084,-3.8326573673$ C,-1.5997701481,-1.6104644662,-3.1731903011 H,-1.7784204008,-1.9882274449,-4.1848250005 $\mathrm{H},-1.951179689,-0.5813852203,-3.1118447045$ H,-2.1856303532,-2.224008403,-2.481756785 $\mathrm{H}, 0.6334714064,-1.2998625557,-4.8381545319$ $\mathrm{H}, 1.8157151071,-0.9557550971,-3.5650249104$ $\mathrm{H}, 0.4691605181,0.1638611428,-3.8422060872$

$\mathrm{HF}=-556.9051879 \mathrm{NImag}=0$
$\mathrm{N},-1.4397299883,-0.4254289916,-0.7641941864$ C, $1.8182665358,0.1277996775,1.7167181453$ $\mathrm{N}, 0.7534477724,0.3102267915,-0.4825055443$ $\mathrm{N},-0.6212626157,-0.0984150216,1.3812613249$ C, $-1.9423306863,-0.4143951896,1.935445438$ C, $1.8293334104,0.8375323327,0.372492092$ $\mathrm{H}, 1.7100047189,1.920706672,0.4937894913$ H,2.771259066,0.6653359032,-0.1491108442 $\mathrm{H}, 0.349153531,-0.4254251306,3.1959403135$ $\mathrm{H},-1.9033037424,-0.2658917228,3.0120335376$ H,-2.2195374613,-1.4574748632,1.7529543237 $\mathrm{H},-2.7135747638,0.2526935064,1.5400484399$ H,2.0779871219,-0.9253258319,1.5794790081 C, $0.4413870547,0.2528439043,2.3462360738$ H, 2.5639470846,0.5692100243,2.3801682478 H,0.266550058,1.2713686953,2.7102065915

$H F=-478.6782083$ NImag=0

\section{ITBDH+}

tbd+ipr.log.acs

Brutoformula: C10H20N3(1+)

1,1 C,- $-0.5361997724,2.277571983,-1.1320584873$ $\mathrm{N},-0.2868633546,0.9193894352,-1.6310663925$ C,-0.0421576503,-0.1505431982,-0.8492955128 H,1.1824565856,-3.0616904705,1.3434469827 $\mathrm{H}, 1.2666825602,-3.0823229138,-1.226650018$ H, 2.1076876823,-1.7537247853,0.6055441557 C, $0.5221433631,-2.5493550592,-0.6309473181$ H,-1.11870702,1.4372874204,1.4908215523 $\mathrm{N}, 0.0743067927,0.0238257086,0.4829890867$ $\mathrm{N}, 0.0869773848,-1.3695088904,-1.4128932166$ C, $0.1999921501,-1.1381780269,1.3745241685$ C,-0.2863216488,-1.6906584802,-2.8314555031 $\mathrm{H},-0.3392933504,-3.214421614,-0.5049586004$ H,0.6241894812,-0.7833040031,2.3137144395 C, $1.1029255066,-2.1693850149,0.719864786$ H, $-0.7900026732,-1.5555026403,1.593474036$ C, $0.2384227357,2.4797049229,0.1604820003$ $\mathrm{H},-1.6095646116,2.4262872615,-0.974144371$ $\mathrm{H},-0.212385811,2.9759320558,-1.9041767511$ $\mathrm{H}, 1.3116470809,2.49810559,-0.0472680563$ C,- $0.0910743365,1.3456295923,1.1201707159$ $\mathrm{H},-0.0293105501,3.4332454185,0.6187270719$ $\mathrm{H}, 0.573918222,1.3720848292,1.9849623832$ $\mathrm{H},-0.2433399425,0.7829964905,-2.6251532349$ H, $-0.1858491396,-2.776566565,-2.855942208$ C, $0.7100925654,-1.1506025973,-3.8687103341$ C,-1.7558328099,-1.3945292962,-3.1598958402 $\mathrm{H}, 0.5370828872,-1.6539496506,-4.8223689908$ $\mathrm{H}, 1.7406938075,-1.3470550139,-3.5663139534$ $\mathrm{H}, 0.6157210302,-0.0782838937,-4.0652346994$ H,-1.999743531,-1.8545413604,-4.1198966542 H,-1.9828163743,-0.3304723636,-3.2443347885 $\mathrm{H},-2.4182121493,-1.8247184624,-2.4056494108$ $\mathrm{HF}=-557.326438 \mathrm{NImag}=0$ 
$\mathbf{M e}_{3}$-Verkade

V.log.acs

\section{Brutoformula: C9H21N4P1}

0,1

N,-1.7023778903,0.1839241128,-0.5104075029

$\mathrm{P},-0.2077630928,0.4367541599,-1.3598150476$

$\mathrm{N}, 0.6480882016,1.6111087151,-0.4096554462$

$\mathrm{N}, 0.6435787802,-1.0896563381,-1.3205599086$

C, $1.3841924868,-1.3447026477,-2.5599957551$

C, $1.279145464,-1.7826228523,-0.2026857521$

C, $-2.5605985064,-0.8556439786,-1.0611877011$

C,-1.9829270941,0.6010565327,0.8529938032

$\mathrm{C}, 0.924575283,1.7458279458,1.0257535222$

C, $0.7875301405,2.9098013504,-1.0761770795$

C, $0.4648937643,-1.8457472947,1.0862823052$

$\mathrm{H}, 1.4707317969,-2.8192610132,-0.5110858293$

$\mathrm{H}, 2.2723547008,-1.3474713912,0.0025097767$

C,-1.2931807125,-0.2687885396,1.9338391708

$\mathrm{H},-3.0688580221,0.5788926845,1.0052406085$

$\mathrm{H},-1.6825020247,1.6438057753,0.9696870496$

H, $1.8637919872,2.3068297402,1.1294421421$

C, $1.0908238459,0.4494365961,1.8364924016$

$\mathrm{H}, 0.1565745926,2.3753360858,1.5016088815$

H,-1.8288128875,-1.2168352493,2.0381063435

$\mathrm{H},-1.3698564879,0.2402176359,2.9078270733$

$\mathrm{H},-0.4485292465,-2.4135056355,0.8972942626$

$\mathrm{H}, 1.1301900587,0.750729504,2.898265553$

$\mathrm{H}, 2.0638161556,0.0106356042,1.6068485326$

$\mathrm{N}, 0.0833247627,-0.5562179905,1.618386705$

$\mathrm{H}, 1.0587850219,-2.4313000728,1.8121228997$

$\mathrm{H}, 0.0958795298,3.6611562517,-0.664825682$

H, $0.5814042669,2.8076882998,-2.1420093144$

$\mathrm{H}, 1.8082805887,3.2938790051,-0.9565554942$

$\mathrm{H},-2.3843724711,-1.8530952888,-0.6305064776$ $\mathrm{H},-2.3941984735,-0.9365056762,-2.1390701997$ $\mathrm{H},-3.6128403722,-0.5935789603,-0.9034657645$ H,2.4393956567,-1.0355242022,-2.4911939398 $\mathrm{H}, 0.9257737508,-0.8014198001,-3.387401914$ H, 1.363686447,-2.4152030664,-2.7961022232

$\mathrm{HF}=-916.0986722 \mathrm{NImag}=0$

\section{ETBD}

etbd.log.acs

\section{Brutoformula: C9H17N3}

0,1

C, $0.4459979379,2.6651386122,-0.2312964435$

N,0.3051228024,1.3684356438,-0.8720614363

C, $0.2535898592,0.2936896274,-0.1650216891$

H, $-0.2111814685,-3.0631157813,1.7859064441$

$\mathrm{H}, 0.0949989466,-3.0015836523,-0.7340585394$

H, $1.3160341463,-2.318317825,1.2818193282$

C,-0.2691039852,-2.1412522057,-0.164895181

$\mathrm{H},-0.8613490997,1.8040488409,2.0569586761$

$\mathrm{N}, 0.3192515067,0.2430409828,1.2362469811$

$\mathrm{N}, 0.2181261429,-0.9359380144,-0.8245221394$

C,-0.1605067654,-0.9167504359,1.9781160102
$\mathrm{Me}_{3}$-VerkadeH+

$\mathrm{V}+1 . \log$.acs

Brutoformula: C9H22N4P1(1+)

1,1

C,-2.5228098919,-0.4600673145,-1.7022066066

$\mathrm{P}, 0.0026771994,0.000995983,-0.7195768862$

$\mathrm{N}, 0.8037544307,1.4858981164,-0.5681541003$

$\mathrm{N}, 0.8860020415,-1.4360682597,-0.5683388074$

$\mathrm{H},-2.0059052856,-0.2921705121,-2.6464334798$

C, $1.3370611856,-1.9060063921,0.7349621916$

$\mathrm{H},-3.4382079042,0.1365928055,-1.7132701072$

C,-2.3171940795,-0.2020861159,0.7323132046

C, $0.9815321259,2.1114719405,0.7358725151$

C, $0.8631624313,2.4203501356,-1.6986083196$

C, $0.3531095665,-1.3882300335,1.7757655579$

H,1.3492560904,-2.9992694429,0.7518439015

H,2.3630188462,-1.570525952,0.9436773216

C,-1.3777041113,0.3895901161,1.7749530069

$\mathrm{H},-3.2695880718,0.3348856356,0.748462164$

$\mathrm{N},-1.6837011769,-0.0474659507,-0.5708467409$

H,1.9197345564,2.672795902,0.7540186098

C, $1.0276822786,1.0003260213,1.7761729307$

$\mathrm{H}, 0.174502568,2.8284304738,0.9451325568$

$\mathrm{H},-1.6023041706,0.0457416729,2.7875525905$

$\mathrm{H},-1.4391086449,1.4776505621,1.7570985171$

$\mathrm{H},-0.5584901534,-1.9851348016,1.7539988348$

$\mathrm{H}, 0.8435119088,1.3644055168,2.7899199134$

H,2.0011682166,0.5105643461,1.7551196668

$\mathrm{N}, 0.0015533717,0.0005139628,1.3841605559$

$\mathrm{H}, 0.7602349159,-1.4114686617,2.7895597073$

$\mathrm{H}, 0.078740819,3.1834917725,-1.633172464$

$\mathrm{H}, 0.7515721726,1.8893056177,-2.6433487757$

H,1.8362239969,2.9173749023,-1.7091225133

$\mathrm{H},-2.7943679019,-1.5202907093,-1.6374633248$

$\mathrm{H}, 0.0034071792,0.0013174751,-2.1268891417$

$\mathrm{H},-2.5412327713,-1.2579980566,0.9411504517$

C, $1.6565951298,-1.9641951484,-1.700667847$

H, $1.5853111654,-3.0546031515,-1.7129351753$

H,2.7138623417,-1.6815870845,-1.6362447538

H,1.2569396253,-1.5945353706,-2.644454247

$H F=-916.5315343$ NImag=0

\section{ETBDH+}

etbd.$+ \log$.acs

Brutoformula: C9H18N3(1+)

1,1

C,-0.4979774653,-0.0335483004,-2.6914347548

$\mathrm{N},-0.5044905375,-0.6525882802,-1.3590141929$

C, $-0.2520628281,0.0059176295,-0.2089532498$

$\mathrm{H}, 0.8967692984,1.7527962471,3.0198868291$

$\mathrm{H}, 0.4570453102,-0.7728541321,2.8702716499$

H, $1.8598622687,0.8962136141,1.8157222004$

C,-0.0451281363,-0.0198177326,2.2597710554

$\mathrm{H},-0.7322039745,2.5847062087,-1.688047705$

$\mathrm{N}, 0.1261592236,1.2980413326,-0.2555598323$

N,-0.3793951183,-0.6458148549,0.9639328143

C,0.2701419733,2.0798287786,0.9826823271 
C, $0.1506079288,-0.928342356,-2.2868786333$ H,-1.3706603764,-2.1953448104,-0.1753758413 $\mathrm{H}, 0.2785484851,-0.8773079543,2.9800967878$ C,0.2281781358,-2.2041558747,1.2709082497 H,-1.2565344004,-0.87787589,2.1079225709 C, $0.96481211,2.5981411529,1.2057585463$ $\mathrm{H},-0.5274156604,3.1773096688,-0.2472067967$ H, $1.1144520783,3.2799233171,-0.8442058044$ $\mathrm{H}, 2.0305078892,2.3474096124,1.210035904$ C,0.1970727816,1.5130354192,1.947574037 $\mathrm{H}, 0.8470903104,3.5611618062,1.7123172459$ $\mathrm{H}, 0.5964584426,1.3673980125,2.9560728313$ $\mathrm{H}, 0.8062445529,-0.1342610577,-2.6397050511$ C,-1.2552202915,-0.7252816283,-2.8642271563 $\mathrm{H}, 0.5610421111,-1.885903454,-2.6261988075$ H,-1.2177802116,-0.7459734324,-3.9577704765 $\mathrm{H},-1.651928009,0.2428148505,-2.5540347625$ H,-1.9464558998,-1.5101431755,-2.542274853

$\mathrm{HF}=-517.5850454 \mathrm{NImag}=0$

\section{TTT}

ttt.log.acs

\section{Brutoformula: C9H14N4}

0,1 $\mathrm{H}, 1.6546102347,1.0392449524,0.8150316495$ C,- $0.3994540575,-0.0790975129,-1.518228171$ C,-1.2912228183,0.5939546249,-0.6046924049 $\mathrm{N}, 0.8904476043,-0.5425083225,-1.1730018947$ C, $0.427021454,-0.8780727263,-3.4218163015$ $\mathrm{N},-0.7197672613,-0.2481515113,-2.762057185$ $\mathrm{H}, 0.9741198399,-0.122406702,-4.0031271197$ H,0.1043751151,-1.6555393034,-4.1184954273 C, $1.2985168415,-1.4213292615,-2.2768117788$ C, $1.2379028928,-0.9734500528,0.1681115414$ $\mathrm{H}, 2.3733811161,-1.351314309,-2.462100662$ H,1.0504732803,-2.4711846976,-2.0473999582 H,1.6970843427,-0.1821921973,2.1005764047 $\mathrm{H}, 2.2779217703,-1.3100144553,0.1337200548$ $\mathrm{H},-0.0756021673,0.5066533764,3.740798616$ $\mathrm{H}, 0.63005129,-1.8308297534,0.5031235736$ C, $-0.4100795144,1.123486247,2.9040927516$ C, $1.1545401325,0.1412439839,1.2079923454$ H,-2.1832718968,0.970226089,-1.0890943358 C,-1.1890981656,0.8196305114,0.7327569149 H,-3.0952214154,1.4813647688,1.1135740613 N,-0.2043025662,0.4328344494,1.629686549 C,-1.921913101,1.3484730967,2.8888438966 $\mathrm{N},-2.1476469838,1.5368111316,1.4567081205$ $\mathrm{H},-2.2256329715,2.2272574356,3.4601104311$ $\mathrm{H}, 0.1293327373,2.0826163185,2.9326074881$ H,-2.4459827025,0.4667405906,3.2828798962

$H F=-570.5172399$ NImag $=0$

\section{PhTMG}

phtmg.log.acs
C,-0.903482509,-2.0245922327,1.0479209976 H,-0.9793862545,0.2480371085,2.7656047436 $\mathrm{H}, 0.9379716136,2.9132834348,0.763628831$ C,0.8433376164,1.1991987455,2.0808334337 H,-0.6997810516,2.4993635672,1.2749151112 C, $0.4996873691,1.1136084491,-2.7000782599$ $\mathrm{H},-1.5037863336,0.3186848644,-2.942360126$ $\mathrm{H},-0.2233197488,-0.804676256,-3.4116597578$ $\mathrm{H}, 1.5183111433,0.7237509398,-2.6249326359$ C,0.2064210027,2.0409548772,-1.5293068459 $\mathrm{H}, 0.4242808335,1.6718014504,-3.6347096504$ $\mathrm{H}, 1.0008822388,2.7806577995,-1.4192717944$ $\mathrm{H},-0.7101933269,-1.6348295091,-1.3009530526$ H,-1.3946217864,-2.1005373789,2.0198027347 C, $0.1693308259,-3.1079941313,0.9174678001$ H,-1.7013436831,-2.1537665065,0.3118302691 $\mathrm{H},-0.2876181089,-4.0933595966,1.0331479158$ $\mathrm{H}, 0.9325235139,-3.0016662692,1.6913694656$ H,0.6720666314,-3.0807998687,-0.0525063239

$\mathrm{HF}=-518.005318 \mathrm{NImag}=0$

\section{TTTH+}

$\mathrm{ttt}+$.log.acs

Brutoformula: C9H15N4(1+)

1,1

$\mathrm{N},-0.8150299041,-0.0426351193,-2.7338050625$

C,- $0.4459560103,-0.026857745,-1.4257498233$

C,- $0.5386525616,1.124859184,-0.6405457766$

$\mathrm{N},-0.0219935237,-1.265710177,-1.0733570825$

C,-0.4132086284,-1.2967431407,-3.3816891363

$\mathrm{H},-0.8779161739,0.818900779,-3.2546393326$

$\mathrm{H}, 0.544924391,-1.1800738122,-3.8986767446$

$\mathrm{H},-1.1653741101,-1.6337722371,-4.0937696228$

C,-0.2871118012,-2.2226499701,-2.1654158475

C, $0.0976780381,-1.7379348256,0.2982734281$

$\mathrm{H}, 0.52909455,-2.9393782064,-2.2576145927$

H,-1.2193742607,-2.7660029428,-1.9718303093

C, $0.9858546675,-0.8491703312,1.1682372863$

H, $0.5566892118,-2.7270137474,0.2510502286$

$\mathrm{H}, 0.5325714475,0.4351407962,3.6785628855$

$\mathrm{H},-0.8891307042,-1.850325517,0.7636501456$

C, $0.7437452068,1.0674563105,2.8160404842$

H, 1.2085717762,-1.3939874792,2.087357357

$\mathrm{H},-0.9306425538,2.0017384288,-1.1383620049$

C, $-0.1889753933,1.2959226755,0.7012863293$

H,- $0.9695406152,3.1832735784,0.9784209668$

N,0.3434502496,0.3955586352, 1.5644980007

C,-0.1080332321,2.3428714103,2.7842906424

$\mathrm{N},-0.3387338824,2.4851088618,1.3418811661$

$\mathrm{H}, 0.4125795573,3.2094358193,3.1897979002$

$\mathrm{H}, 1.8158319422,1.2956537162,2.794641765$

$\mathrm{H},-1.0532700791,2.2080290745,3.3202330277$

H, 1.9366576342,-0.6561516187,0.656553681

$\mathrm{HF}=-570.9511961 \mathrm{NImag}=0$

PhTMGH+

phtmg+.log.acs 


\section{Brutoformula: C11H17N3}

0,1

$\mathrm{N}, 0.0879739889,0.6922620182,-1.2751398188$

C, $0.8631186789,-0.0847681421,-0.4173445755$

$\mathrm{N}, 2.2334896399,0.0067705677,-0.642697132$

$\mathrm{N}, 0.435428004,-0.8747427343,0.5108449604$

C, $-0.7953590948,-0.7279025283,1.1539471193$

C, $0.4471861367,2.0651397688,-1.6018335432$

C,-1.2731578432,0.3112946675,-1.6169731431

C,3.1188020088,-0.5635988947,0.365158603

C, $2.7566730485,-0.0917531548,-2.0034067416$

C,-3.2135076643,-0.551396949,2.6021096977

C,-1.5769074058,-1.8699702725,1.4005626133

C,-1.2409389247,0.5022395588,1.6730720744

C,-2.4348639903,0.5849006883,2.385601745

C,-2.7726324678,-1.7790181316,2.1052040449

$\mathrm{H},-1.2235512575,-2.8253717659,1.0293276635$

$\mathrm{H},-0.6319537645,1.3887066067,1.5308582496$

$\mathrm{H},-2.7539232478,1.543597803,2.7814523286$

$\mathrm{H},-3.3606074523,-2.6747492756,2.2757923657$

$\mathrm{H},-4.140700363,-0.4842170327,3.1593446753$

$\mathrm{H},-1.4488864766,0.5429621538,-2.6734786068$

$\mathrm{H},-2.0241256755,0.8377127312,-1.015400377$

H,-1.4098215176,-0.7584934189,-1.4718347713

$\mathrm{H}, 0.4417218485,2.2302153203,-2.6863619691$

$\mathrm{H}, 1.439603743,2.2923445572,-1.2180879498$

H,-0.2696946516,2.7657650151,-1.151636011

H,3.0293460334,-1.1295778354,-2.2437420404

H,3.6511185041,0.5290271677,-2.1099060362

H,2.0127683979,0.2405222428,-2.7233395351

$\mathrm{H}, 4.1145897401,-0.1290248138,0.2406433934$

H,3.1945327649,-1.6554471727,0.2771150143

$\mathrm{H}, 2.7442792627,-0.333428745,1.3601477017$

$H F=-593.8013961$ NImag $=0$

\section{H2NNH2}

hy1.log.acs

\section{Brutoformula: H4N2}

0,1

$\mathrm{H}, 1.1722280517,0.7416835296,-0.475374271$

$\mathrm{N}, 0.7053578001,-0.1187490492,-0.2154478363$

$\mathrm{H}, 1.0553165829,-0.4187079655,0.6908221073$

$\mathrm{N},-0.7053578001,0.1187490492,-0.2154478363$

$\mathrm{H},-1.1722280517,-0.7416835296,-0.475374271$

H,-1.0553165829,0.4187079655,0.6908221073

$H F=-111.9105742$ NImag $=0$

\section{NCNH2}

nh2cn.log.acs

\section{Brutoformula: C1H2N2}

0,1

$\mathrm{N},-0.1730670376,0.0001321294,-0.5768323229$

C,-0.0516514957,0.0004643948,0.7577602001
Brutoformula: C11H18N3(1+)

1,1

$\mathrm{N}, 0.0935620691,0.828997433,-1.2386501728$

C,-0.1933507733,-0.4339190827,-0.8771290625

$\mathrm{N},-0.0062201172,-1.4586457314,-1.7329123828$

$\mathrm{N},-0.6995582144,-0.7040921281,0.3487481731$

C,-0.3932344072,-0.0402400849, 1.5853171908

C, $1.2261715433,1.1563888979,-2.1146823462$

C,-0.6176483203,1.9860065757,-0.6779843648

C, $-0.1863716831,-1.3113343984,-3.184391477$

C, $0.1963031086,-2.8366861421,-1.264203992$

C,0.1641484466,1.1603644644,4.0327584387

C,-1.4178285586,0.1474670505,2.5129229522

C, $0.9115589924,0.3585986729,1.8774164327$

C,1.1819985415,0.9700339998,3.0996287706

C, $-1.1324652254,0.741228647,3.739947537$

H,-2.4296349093,-0.165043851,2.2781807887

$\mathrm{H}, 1.7132847655,0.1785241718,1.170310272$

H,2.1948181818,1.2789875523,3.3289910219

H,-1.9269802198,0.8812263299,4.4628765956

$\mathrm{H}, 0.3821438152,1.6261299199,4.985981193$

$\mathrm{H},-1.5594083431,1.6730230451,-0.234675069$

$\mathrm{H},-0.827027802,2.6821724306,-1.492635972$

$\mathrm{H},-0.0148571687,2.4933457769,0.0790621568$

$\mathrm{H}, 0.8864817663,1.5550181802,-3.0736240849$

$\mathrm{H}, 1.8428268909,0.2770918817,-2.2850739426$

$\mathrm{H}, 1.8352091493,1.9168264373,-1.6197897832$

H,-0.7357814174,-3.4104797828,-1.2917482509

$\mathrm{H}, 0.5999979405,-2.839479943,-0.25343463$

$\mathrm{H}, 0.9158629949,-3.3243067713,-1.9239833481$

H,-0.6281664732,-0.3449654597,-3.4144336989

$\mathrm{H},-0.8661585758,-2.0932667422,-3.5302389187$

$\mathrm{H}, 0.7625030471,-1.4152466062,-3.71586224$

H,-1.2721790433,-1.5337247431,0.4233122146

$\mathrm{HF}=-594.2097857 \mathrm{NImag}=0$

\section{H2NNH2H+}

Hy1+.log.acs

Brutoformula: H5N2(1+)

1,1

$\mathrm{H}, 0.2197118218,-0.8312829553,1.2503015465$

N,-0.2302166247,0.,0.8720303006

$\mathrm{H}, 0.2197118217,0.8312829553,1.2503015465$

$\mathrm{N},-0.054405858,0 .,-0.5643474728$

$\mathrm{H},-0.5360903066,0.8211142494,-0.949351041$

$\mathrm{H},-0.5360903066,-0.8211142494,-0.949351041$

H,0.9173794513,0.,-0.9095838378

$\mathrm{HF}=-112.2504799 \mathrm{NImag}=0$

\section{NCNH2H+}

$\mathrm{Nh} 2 \mathrm{cn}+. \log$.acs

Brutoformula: C1H3N2(1+)

1,1

H,-0.2795089032,0.8750905211,1.5022317897

N,-0.1980706904,0.0000000046,0.989410334 
$\mathrm{N}, 0.0004355137,-0.0005467713,1.9144013911$ $\mathrm{H}, 0.111989897,0.8477472846,-1.0480256595$ H,0.1122931217,-0.8477970376,-1.0473036098

$\mathrm{HF}=-148.8349251$ NImag $=0$

\section{(CF3)3CNH2}

cf33nh21.log.acs

Brutoformula: C4H2F9N1

0,1

C, $0.9280476278,1.2092380767,0.3352489883$

C, $0.1033860519,-0.0439935833,-0.1572481638$

C, $0.7866724953,-1.3590063279,0.3537466127$

$\mathrm{N}, 0.0295883183,-0.1123947953,-1.5945127038$

C, $-1.3636590218,0.0455423002,0.388909198$

F, $2.0668037874,1.3135125649,-0.3753406025$

F, $1.2565657487,1.17309593,1.6307229783$

F, $0.2224055013,2.3350301579,0.1131832928$

F,-1.3973071375,0.439127897,1.6695379507

F, $-1.9943634379,-1.1293130516,0.2994034056$

F,-2.0682697357,0.9375348711,-0.3345007983

F,0.6891711458,-1.4899401826,1.6834197582

F, 2.0979569384,-1.3433422696,0.031891188

F, $0.2541302462,-2.4429222651,-0.21184491$

$\mathrm{H},-0.4443075984,0.6923583952,-1.9874248664$

H,0.9464938492,-0.2060250086,-2.0159256668

$\mathrm{HF}=-1107.3139711 \mathrm{NImag}=0$

\section{CF3CH2NH2}

cf3nh2.log.acs

Brutoformula: C2H4F3N1

0,1

F, $0.8536378389,1.0875313994,1.2214728817$

C, $0.0666989708,0 ., 1.0376248267$

F, $0.8536378387,-1.0875313997,1.2214728816$

C, $-0.5905799057,0 .,-0.3369893207$

F, $-0.8552834485,-0.0000000001,2.0262300705$

$\mathrm{N}, 0.319693257,-0.0000000002,-1.4632793945$

$\mathrm{H},-1.235618747,0.8809682538,-0.3810785544$

$\mathrm{H},-1.2356187472,-0.8809682535,-0.3810785544$

$\mathrm{H}, 0.9117164695,0.8226755334,-1.4721874144$

$\mathrm{H}, 0.9117164705,-0.8226755331,-1.4721874132$

$\mathrm{HF}=-433.0505996 \mathrm{NImag}=0$

\section{$\mathrm{PhCH}_{2} \mathrm{NH}_{2}$}

bznh2.log.acs

\section{Brutoformula: C7H9N1}

0,1

C, $0.5046780017,-0.2703711312,-1.7715988187$

C, $0.2387315361,-0.0363674321,-0.2968193829$

C, $-0.1909078267,0.4409438273,2.438156539$

C,0.9841301461,-0.7134336521,0.6725101992
$\mathrm{H},-0.2795089539,-0.87509049,1.502231818$

C, $0.0158006725,-0.0000000241,-0.2737899843$

$\mathrm{N}, 0.1095392403,-0.0000001254,-1.4268663342$

$\mathrm{H}, 0.6317469117,0.0000001138,-2.2932181532$

$H F=-149.1532585$ NImag $=0$

\section{(CF3)3CNH2H+}

cf33nh2+10.log.acs

Brutoformula: C4H3F9N1(1+)

1,1

C, $-1.5081383585,-0.0282682167,0.5026962321$

C, $0.0000091654,0.0002640746,0.0416830238$

C, $0.7303615647,1.3199464443,0.5025110574$

N,0.00084309,0.0006174173,-1.4745945637

C, $0.776767672,-1.2920218705,0.5042345136$

F,-2.0651831911,1.1670631412,0.3053075988

F,-1.6023267563,-0.3730181671,1.7692350155

F,-2.1670141426,-0.9174627632,-0.2650311109

F, 1.1204984142,-1.2009885441,1.7711208909

F, 1.8774643392,-1.4206646216,-0.2612191448

F, $0.0181219989,-2.3709617769,0.3062458339$

F, $0.4849442228,1.57139295,1.7705059524$

F, $0.2869165308,2.336916745,-0.261243781$

F,2.0433848789,1.2045815691,0.2983136602

$\mathrm{H}, 0.9609202637,-0.0891304815,-1.8359308741$

H,- $0.4009258264,0.8770767993,-1.8361427603$

H,- $0.5566438647,-0.7853426984,-1.837691563$

$H F=-1107.6193989$ NImag $=0$

\section{CF3CH2NH2H+}

cf3nh $2+. \log$.acs

Brutoformula: C2H5F3N1(1+)

1,1

F,0.8525360878,1.0874694991,1.3298192328

C, $0.0613614108,0 ., 1.2421424531$

F, $0.8525360874,-1.0874694995,1.3298192327$

C,-0.6634339491,0.0000000001,-0.1044062039

F,-0.8122924045,0.,2.2334008827

$\mathrm{N}, 0.3726306528,0 .,-1.2053804649$

$\mathrm{H},-1.2753575632,0.8956119357,-0.2040901253$

H,-1.2753575634,-0.8956119353,-0.2040901254

$\mathrm{H}, 0.9772501495,0.8289564818,-1.1426334366$

$\mathrm{H},-0.0671230474,-0.0000000007,-2.1319478797$

H,0.9772501503,-0.8289564811,-1.1426334357

$H F=-433.3804451$ NImag=0

\section{$\mathrm{PhCH}_{2} \mathrm{NH}_{2} \mathrm{H}+$}

bznh2+.log.acs

Brutoformula: C7H10N1(1+)

1,1

C,0.6752384536,0.000000001,-1.6234546075

C, $0.3819645605,0.0000000007,-0.1552582763$

C, $-0.2229843313,-0.0000000001,2.5679305766$

C, $0.2349816316,-1.2117864289,0.5324564476$ 
C,-0.73259178,0.8798668727,0.1221099398

C, $-0.9454639095,1.1160077871,1.4783500361$

C,0.7757049295,-0.4758910778,2.0309650161

$\mathrm{H}, 1.733855299,-1.4352727039,0.3626982403$

H,-1.3232389668,1.3950748022,-0.6261070479

$\mathrm{H},-1.7024010645,1.8287370389,1.7875890729$

$\mathrm{H}, 1.3629393194,-1.0117345004,2.7686959625$

H,-0.3585775696,0.6248592387,3.4935346395

H,1.107234692,-1.1849563038,-1.8766608989

$\mathrm{H}, 1.1202141444,0.5513155265,-2.1568262784$

$\mathrm{N},-0.7346523129,-0.2848121221,-2.5578815274$

$\mathrm{H},-0.5334734052,-0.3349778739,-3.5505886443$

$\mathrm{H},-1.3061812331,-1.0889882993,-2.3181270468$

$\mathrm{HF}=-327.0026519 \mathrm{NImag}=0$

\section{4-MeO-Aniline}

meo.log.acs

\section{Brutoformula: C7H9N1O1}

0,1

N, $0.431487577,-0.0366559837,-3.2652729075$

C, $0.3640169924,0.0037053163,-1.8609468758$

C, $0.2427081307,-0.046217196,0.9579287701$

C, $1.5352395739,-0.0167425338,-1.0866368111$

C, $-0.8619320549,-0.0046869627,-1.1937582415$

C,- $-0.9287334301,-0.0291054699,0.2017388642$

C, $1.4760131246,-0.0392142242,0.298135601$

$\mathrm{H}, 2.50269413,-0.0199870244,-1.5790179606$

$\mathrm{H},-1.7847376762,0.0028299899,-1.7653818103$

H,- $1.9007898152,-0.0334640145,0.6767840286$

$\mathrm{H}, 2.3825573361,-0.0526672576,0.8915922325$

$\mathrm{O}, 0.2928630958,-0.0691022159,2.3294223911$

C, $-0.9311472293,-0.081244493,3.0440127149$

$\mathrm{H},-1.5254443361,0.8174217583,2.8404320601$

$\mathrm{H},-0.6626370364,-0.1018212226,4.0993339505$

$\mathrm{H},-1.5277669883,-0.969840491,2.8060708758$

$\mathrm{H},-0.383720003,0.3283247685,-3.7373420394$

H,1.279328608,0.3484672566,-3.6570948415

$\mathrm{HF}=-402.2406752 \mathrm{NImag}=0$

\section{2-Me-Aniline}

mean.log.acs

\section{Brutoformula: C7H9N1}

0,1

$\mathrm{N},-0.0000000001,0.1476631582,-3.0251479494$

C, $0 ., 0.0586459443,-1.6265377284$

C, $0.0000000002,-0.0045315319,1.2132225933$

C, $1.2016193701,0.0418648965,-0.9063407048$

C,-1.2016193699,0.0418648962,-0.9063407047

C,-1.193639998,0.0092089478,0.4846576441

C, $1.1936399983,0.0092089482,0.484657644$

$\mathrm{H}, 2.1470199823,0.0543494342,-1.4401661632$

H,-2.1470199822,0.0543494336,-1.4401661631

$\mathrm{H},-2.1417235619,-0.0083479309,1.0136464812$

H,2.1417235623,-0.0083479297,1.0136464811
C, $0.2349816334,1.2117864298,0.5324564486$

C, $-0.0683042548,1.2094321697,1.8909718986$ C,-0.0683042563,-1.2094321695,1.8909718977 $\mathrm{H}, 0.3814413538,-2.1564182235,0.0170108978$ $\mathrm{H}, 0.3814413567,2.1564182247,0.0170108995$ $\mathrm{H},-0.1706285552,2.1476471697,2.4225610109$ $\mathrm{H},-0.1706285579,-2.1476471698,2.4225610092$ $\mathrm{H},-0.4512113216,-0.0000000003,3.6270821465$ N,-0.6495349358,-0.0000000012,-2.4266305572 H, 1.21234149,-0.8893649974,-1.9523730993

H,1.2123414876,0.8893650007,-1.9523730996 $\mathrm{H},-1.2092524661,-0.8234637855,-2.1854551954$ H,-0.494630782,-0.0000000022,-3.4400131396 $\mathrm{H},-1.2092524683,0.8234637821,-2.1854551969$

$H F=-327.3681879$ NImag $=0$

\section{4-MeO-Aniline $\mathrm{H}+$}

meo+.log.acs

Brutoformula: C7H10N1O1(1+)

1,1

N,-0.40872121,-0.0217368674,-3.1102231144

C, $-0.3380037722,-0.0025689529,-1.6184327049$

C,- $0.2075109232,0.0532036253,1.1424978236$

C,-1.5167680799,0.1652125286,-0.8939065047

C, $0.8938212479,-0.1413225474,-1.0027614151$

C, $0.9634769593,-0.1144454696,0.388183643$

C,-1.4467067234,0.1918542132,0.4857277066

H,-2.4770914287,0.2734028394,-1.3874290678

$\mathrm{H}, 1.8049844421,-0.2711835756,-1.5783030277$

$\mathrm{H}, 1.9255148203,-0.2257111226,0.8674209281$

$\mathrm{H},-2.3388026057,0.3182166579,1.0855417974$

$\mathrm{O},-0.256737945,0.0932423458,2.4791044857$

C, $0.9512526283,-0.0350248853,3.243773214$

H, $1.4236158653,-1.0042670378,3.0636458732$

$\mathrm{H}, 0.6421248555,0.0329039645,4.2834088595$

H,1.6445359001,0.7778882921,3.0126477596

$\mathrm{H}, 0.5053659812,-0.2516670734,-3.5113714147$

H,-0.690662788,0.8862180807,-3.4973531675

H,-1.0736872229,-0.7242150124,-3.4521716729

$H F=-402.5957004$ NImag=0

2-Me-Aniline $\mathrm{H}^{+}$

mean+1.log.acs

Brutoformula: C7H10N1(1+)

1,1

$\mathrm{N}, 0.0005386035,0.0026582762,-2.8755674948$

C, $0.0000389038,-0.015103813,-1.3798925042$

C, $-0.0006377862,0.0171578816,1.3949160383$

C,1.2168272343,-0.0056536424,-0.7144982142

C, $-1.2167625753,-0.0060957951,-0.715288954$

C,-1.2037325372,0.0099335129,0.6763957787

C, $1.2031457322,0.0103501408,0.6767566325$

H,2.159905201,-0.0090420249,-1.2514593168

$\mathrm{H},-2.1596412302,-0.0098100755,-1.2525932311$

H,-2.1473801846,0.016403451,1.2091208062

$\mathrm{H}, 2.1466151347,0.017079228,1.2099555507$ 
C, $-0.0000000001,-0.005384625,2.7235558461$ $\mathrm{H}, 0.0000000025,1.0146717485,3.1246395339$ $\mathrm{H}, 0.8832083767,-0.5111305935,3.1221676014$ $\mathrm{H},-0.8832083798,-0.5111305887,3.1221676005$ $\mathrm{H},-0.8358420569,-0.1914771037,-3.4788310074$ H,0.8358420566,-0.1914771041,-3.4788310076

$\mathrm{HF}=-327.0141687$ NImag $=0$

\section{4-CF3-Aniline}

cf3an.log.acs

\section{Brutoformula: C7H6F3N1}

0,1

H,-2.1505000723,0.0485807389,-1.4518822028

C, $-1.2075051893,0.0336480765,-0.9156772871$

C, $1.2048829978,-0.0022715298,0.4705754059$

C, $0.0000000001,0.0520631675,-1.6336081974$

C,-1.2048829981,-0.0022715289,0.4705754058

C, $-0.0000000002,-0.0239731438,1.1765258104$

C, $1.2075051893,0.0336480758,-0.9156772869$

$\mathrm{N}, 0.0000000005,0.1373453449,-3.0182567783$

$\mathrm{H},-2.1455966753,-0.0225671696,1.0069724607$

C,0.0000000001,-0.0084947271,2.6727944277

$\mathrm{H}, 2.1505000724,0.0485807379,-1.4518822025$

$\mathrm{H}, 2.1455966749,-0.0225671712,1.0069724609$

$\mathrm{H},-0.8432500288,-0.1433194173,-3.4946068004$

$\mathrm{H}, 0.8432500283,-0.1433194216,-3.4946068004$

F,- $0.0000000044,1.256848515,3.1801476341$

F,1.0894051096,-0.6209652706,3.1958169736

F,-1.0894051046,-0.6209652785,3.1958169747

$H F=-624.8398366$ NImag $=0$

\section{Pyrrolidine}

pyrr.log.acs

Brutoformula: C4H9N1

0,1

H,-1.5537709915,0.000000013,-1.1724382281

$\mathrm{N},-0.5460424918,0.0000000061,-1.3034518693$

C, $0.0345344846,1.1612341283,-0.6132227622$

C, $0.0345344675,-1.1612341266,-0.6132227631$

$\mathrm{H}, 1.0240645889,1.3530066677,-1.0426250346$

C, $0.1684356028,0.7774306711,0.8855287134$

$\mathrm{H},-0.5735136886,2.0520630864,-0.7820347483$

H, $1.0240645688,-1.3530066804,-1.0426250356$

$\mathrm{H},-0.5735137195,-2.0520630749,-0.782034751$

C,0.1684355934,-0.7774306758,0.8855287131

$\mathrm{H}, 1.075051433,1.1931725161,1.3317585519$

$\mathrm{H},-0.6786656415,-1.1634716907,1.45854033$

$\mathrm{H},-0.6786656253,1.1634716916,1.4585403369$

H,1.0750514204,-1.193172534,1.3317585469

$\mathrm{HF}=-212.6460428 \mathrm{NImag}=0$
C, $0.0006991913,0.0036541576,2.9000067857$

$\mathrm{H},-0.90794408,0.451572644,3.3044862325$

$\mathrm{H}, 0.8602664242,0.5420671244,3.3023828816$

$\mathrm{H}, 0.0543733416,-1.0256453865,3.269867511$

$\mathrm{H}, 0.0008820134,0.9578658896,-3.2525846935$

$\mathrm{H},-0.8244277268,-0.475968842,-3.2512398014$

$\mathrm{H}, 0.8255019483,-0.4763563296,-3.2507634271$

$H F=-327.3656369$ NImag $=0$

\section{4-CF3-Aniline $\mathrm{H}+$}

cf3an+1.log.acs

Brutoformula: C7H7F3N1(1+)

1,1

H, $0.1210237591,2.1578926205,-1.2645512352$

C, $0.0624968157,1.2179487351,-0.7263579051$

C,-0.0969606252,-1.2107693417,0.6647368858

C, $-0.0078942515,-0.0003382167,-1.3868527344$

C,0.0529617804,1.2093595147,0.664979364

C, $-0.026646843,-0.0017235223,1.3537796665$

C, $-0.0883626664,-1.2170655641,-0.7284384318$

$\mathrm{N}, 0.0186083876,0.0007366625,-2.8831675561$

$\mathrm{H}, 0.0980959192,2.144200315,1.2087886477$

C, $0.0101137975,-0.0008497619,2.8711685102$

$\mathrm{H},-0.1467978349,-2.1568046959,-1.2672735173$

$\mathrm{H},-0.1681404393,-2.1450119519,1.2064435087$

H,-0.6088254746,0.7143496623,-3.2700355644

$\mathrm{H},-0.2843399401,-0.9046328575,-3.2565505369$

$\mathrm{H}, 0.9607513215,0.1840187918,-3.2486481726$

F,-0.5702654034,1.10276689,3.3717181041

F, 1.2844817966,-0.0288796953,3.3091161296

F,-0.6212356823,-1.0728130827,3.3773319261

$\mathrm{HF}=-625.1714314 \mathrm{NImag}=0$

\section{Pyrrolidine $\mathbf{H}+$}

pyrr+.log.acs

Brutoformula: C4H10N1(1+)

1,1

$\mathrm{N}, 0 ., 0 .,-1.2619195142$

$\mathrm{H}, 0.086353647,0.8119806784,-1.8746232757$

C, $1.2379566761,-0.1391382823,-0.3548693015$

C, $-1.2379566761,0.1391382823,-0.3548693015$

H, $1.5456137549,-1.1832000651,-0.4022852209$

C, $0.7264555108,0.247978124,1.031825785$

H, 2.033710886,0.4881175614,-0.7520570498

$\mathrm{H},-2.0337108861,-0.4881175613,-0.7520570498$

$\mathrm{H},-1.5456137549,1.1832000651,-0.402285221$

C,- $0.7264555108,-0.2479781239,1.031825785$

$\mathrm{H}, 1.332946822,-0.2118092682,1.8120038134$

H,-1.332946822,0.2118092682,1.8120038134

$\mathrm{H}, 0.7690465231,1.3314220984,1.1709653126$

$\mathrm{H},-0.769046523,-1.3314220984,1.1709653125$

$\mathrm{H},-0.086353647,-0.8119806785,-1.8746232756$

$H F=-213.0216821 \quad$ NImag $=0$

\section{Et3N H+}


et3n.log.acs

Brutoformula: C6H15N1

0,1

C, $-1.3303286476,-0.1161607754,-0.2673058628$

$\mathrm{N}, 0.0647480776,-0.2312967453,-0.6778689669$

C, $0.8989500746,0.9575577267,-0.512853018$

C, $0.6855382837,-1.5212758146,-0.3917710992$

H, $1.6634926605,-1.5306072129,-0.8850333847$

$\mathrm{H},-1.7427989936,1.9645946653,-0.8162201123$

C, $0.8614454568,-1.9440993697,1.0805115664$

H, $1.8971566448,0.6997520008,-0.8804647048$

C, $1.0222881641,1.575760213,0.8938277201$

$\mathrm{H}, 0.5236796539,1.7321228126,-1.1909815319$

H,-1.4468511863,0.0677440577,0.8153694045

H,-1.7982052942,-1.0876197748,-0.4591744157

C, $-2.1028257752,0.9566484505,-1.0378145342$

H,-3.1615218365,0.9221132349,-0.7652951463

H,-2.0165756146,0.7938475497,-2.1153043875

$\mathrm{H}, 0.0826431357,-2.2862214221,-0.8964093457$

H,1.2510659136,-2.9663520817,1.1257089694

H,-0.089562341,-1.9299494469,1.6201756816

H,1.5631659587,-1.2992809392,1.6139113536

H,1.6469850523,2.4734357826,0.8446144197

H, $1.4782756031,0.8910156873,1.6114202825$

$\mathrm{H}, 0.0492350067,1.8782714015,1.2909571162$

$\mathrm{HF}=-292.4971809 \mathrm{NImag}=0$

\section{NCNMe2}

me2ncn.log.acs

\section{Brutoformula: C3H6N2}

0,1

C, $0.0624561932,-1.2480018655,-0.4351651979$

$\mathrm{N},-0.3182622619,0.0000392947,0.2348984521$

C, $-0.3254342873,0.0000178182,1.5682998421$

C, $0.0624409979,1.2480542868,-0.4352046765$

$\mathrm{N},-0.3697686077,-0.0002601609,2.7287436645$

H,-0.3602553167,2.0926676656,0.1074902308

H,-0.3475680794,1.2407272272,-1.4469981639

H, $1.152076825,1.3672452029,-0.4913622221$

H,1.1520943103,-1.3671476881,-0.4913658425

H,-0.3476042997,-1.2407432228,-1.4469389125

H,- $0.3601754745,-2.0925985584,0.1076028248$

$H F=-227.4680452$ NImag $=0$

\section{Ph3N}

ph3n.log.acs

Brutoformula: C18H15N1

0,1

C, $0.0000604582,-0.0477803988,1.4186079579$

$\mathrm{N}, 0.0001090573,0.00542509,-0.0018618622$

C,-1.2298026534,0.079702429,-0.7097321843

C, $1.2304491683,-0.0230396738,-0.7127025105$

H,-2.6520856532,1.6466982267,-3.3728322769 et3n+.log.acs

Brutoformula: C6H16N1(1+)

1,1

C, $0.7122026763,1.047470964,-0.28526823$

$\mathrm{N}, 0.0559569693,-0.1620368663,0.3652874003$

C, $0.4451722226,-1.465397648,-0.3237556347$

C,-1.4540624346,-0.0285289584,0.547438595

$\mathrm{H}, 0.4393970538,-0.2171244058,1.3127004178$

$\mathrm{H}, 0.2680549956,1.1459069379,-1.2751432469$

$\mathrm{H}, 1.7615222781,0.784694599,-0.4123024787$

C, $0.5754259819,2.3204447211,0.5358159639$

$\mathrm{H},-1.612502235,0.8567780504,1.1620500393$

$\mathrm{H},-1.7512722806,-0.8987385642,1.1351835275$

C,-2.2372855366,0.0492345676,-0.753110275

C, $1.8500479453,-1.9224162502,0.0403182759$

$\mathrm{H}, 0.3327578858,-1.3003182357,-1.3950274259$

H,-0.2918262089,-2.2066648519,-0.0132207109

$\mathrm{H},-2.002050755,0.9413541572,-1.3358028497$

$\mathrm{H},-3.2985081084,0.1011317418,-0.5012778132$

$\mathrm{H},-2.0964726008,-0.8325724408,-1.3799928167$

H,1.171631877,3.1012056941,0.0594412858

$\mathrm{H}, 0.9617929839,2.1946541405,1.5515166202$

$\mathrm{H},-0.4510767428,2.6849627323,0.5901253891$

H, 2.0370442424,-2.8832078397,-0.4432794457

$\mathrm{H}, 1.9585682757,-2.0764357364,1.1179099158$

H,2.6254815129,-1.2343965077,-0.2996065042

$H F=-292.8866221$ NImag=0

\section{NCNMe2H+}

me2ncn+.log.acs

Brutoformula: C3H7N2(1+)

1,1

C, $0.1572475487,-1.2831800161,-0.7247532913$

$\mathrm{N}, 0.0219490945,0.0003271653,0.0262652338$

C,- $0.2032752557,-0.000027613,1.2714585574$

C, $0.1536119064,1.2836240536,-0.7254472481$

$\mathrm{N},-0.2528902978,-0.000661005,2.4507282147$

H,-1.0119225736,-0.0014944622,3.1246475932 $\mathrm{H}, 0.0464345825,2.1214763396,-0.0396356147$

$\mathrm{H},-0.6261921079,1.3178460195,-1.4860100088$

H,1.140704489, 1.3036139299,-1.1871582741

H, $1.1447877495,-1.3010921391,-1.1855861382$

$\mathrm{H},-0.6218449542,-1.3195391125,-1.4859369625$

$\mathrm{H}, 0.0513898106,-2.12089316,-0.0385720614$

$\mathrm{HF}=-227.8084468$ NImag $=0$

\section{Ph3N H+}

ph3n+.log.acs

Brutoformula: C18H16N1(1+)

1,1

C,1.2821916011,0.6941090857,0.2710172592

$\mathrm{N}, 0 ., 0 ., 0.6445156893$

C,-0.0399796993,-1.4574650419,0.2710172592

C,-1.2422119018,0.7633559562,0.2710172592

H,0.,0.,1.6675422605 
C,-2.5674311144,0.9876781843,-2.5157125351 C,-3.6634156988,0.2279072638,-2.1079899645 C, $-1.3628883902,0.9235108527,-1.8216517694$ $\mathrm{H},-2.2374270771,-1.34795162,0.5470507412$ $\mathrm{H},-0.5196435169,1.5250897229,-2.1386359105$ C,-2.3311254647,-0.6894281474,-0.3078892464 $\mathrm{H},-4.601766648,0.2853402443,-2.6470831794$ H,-4.3774910062,-1.2096101236,-0.6727292158 C,-3.5368182682,-0.6066796928,-0.9980752632 C, $-0.0026241547,-0.1543651849,4.2266303735$ C, $0.8555103193,-0.9254398777,2.0990970926$ C, $-0.8561151833,0.7764447615,2.1622305329$ C,-0.8600931106,0.7145138152,3.5525205172 C,0.8565706524,-0.9693225983,3.4901752906 H, $1.5179509008,-1.5692575548,1.5332170278$ H,-1.5173585085,1.4616433897,1.6457476491 $\mathrm{H},-1.5288246301,1.3600853917,4.1112150928$ $\mathrm{H}, 1.5243130277,-1.6554562761,3.9995306851$ H,-0.00377981,-0.1957078503,5.3095484782 C,3.6653182015,-0.0811543876,-2.1147870998 C, $1.365402684,-0.7957145096,-1.8747545064$ C, $2.3302840082,0.7207480116,-0.2623473453$ C, $3.5367372945,0.6825394939,-0.9551351668$ C, $2.5707047718,-0.8153285402,-2.5703079356$ $\mathrm{H}, 0.5230568565,-1.3773804049,-2.2290283591$ H,2.2345399665,1.3247291862,0.6318183432 $\mathrm{H}, 4.3764140639,1.2649790632,-0.5920801601$ H,2.6570100506,-1.4197366119,-3.4666125136 H,4.6042594089,-0.1036816671,-2.655440774

$\mathrm{HF}=-749.8854119 \mathrm{NImag}=0$

\section{$\mathrm{N}, \mathrm{N}$-dimethylaniline (DMA) \\ dma1.log.acs}

\section{Brutoformula: C8H11N1}

0,1 $\mathrm{N}, 0.0830299421,0.0000050891,-1.5768833126$ C, $-0.0422502408,0.000009582,-0.1902322341$ C, $-0.3713770026,-0.0000093656,2.6298193022$ C, $-0.1160390417,-1.2060288342,0.5408667921$ C,-0.1160191154,1.2060393657,0.5408857287 C, $-0.282554472,1.1966389534,1.9218259792$ C, $-0.2825743878,-1.1966478776,1.9218070921$ H,-0.0449245967,-2.1582704797,0.034079795 $\mathrm{H},-0.04488761,2.1582884975,0.0341178959$ H,- $0.3382307095,2.1436034031,2.4483008392$ $\mathrm{H},-0.3382665518,-2.1436198957,2.4482665743$ H,- $0.498743406,-0.0000168863,3.7056257938$ C, $0.414882278,1.2406325963,-2.2584037522$ C,0.4149011444,-1.2406345788,-2.2583699823 H,1.3926687823,1.6465895676,-1.9571315569 $\mathrm{H},-0.3434781259,2.0053094248,-2.0708514292$ $\mathrm{H}, 0.4357883581,1.0600751492,-3.3327848731$ H,1.3927015247,-1.6465589794,-1.9570992926 $\mathrm{H}, 0.4357867176,-1.0601116908,-3.3327572044$ H,-0.3434389494,-2.0053227812,-2.0707769088

$H F=-366.3140513$ NImag=0
C,3.6078908154,2.042723552,-0.3509033164 C, $1.697065862,1.7364937699,1.0922288951$ C, $1.9988459023,0.3052933638,-0.8510980063$ C,3.1716516882,0.9956655666,-1.1592854411 C, $2.8731471505,2.4112011105,0.7755965673$ $\mathrm{H}, 1.1147837814,2.0307793237,1.959491099$ $\mathrm{H}, 1.6700982266,-0.5198860309,-1.4688363872$ H,3.7451126902,0.7043377005,-2.0307040681 H,3.2119415994,3.2212363305,1.4095788206 H,4.5228724881,2.568778243,-0.5945825731 C,-3.5729958966,2.1031633242,-0.3509033164 C, $-1.2638147599,1.5784046477,-0.8510980063$ C,-2.3523806492,0.6014552634,1.0922288951 C, $-3.5247349906,1.2826178659,0.7755965673$ C,-2.4480975184,2.2488981507,-1.1592854411 H,- $0.3848146035,1.7062905065,-1.4688363872$ $\mathrm{H},-2.3160983745,-0.0499585874,1.959491099$ $\mathrm{H},-4.3956432935,1.1710048553,1.4095788206$ H,-2.4825306865,2.8911938795,-2.0307040681 $\mathrm{H},-4.4860634592,2.6325333512,-0.5945825731$ C,- $0.0348949188,-4.1458868762,-0.3509033164$ C, $-0.7350311425,-1.8836980116,-0.8510980063$ C, $0.6553147872,-2.3379490333,1.0922288951$ C, $0.6515878401,-3.6938189764,0.7755965673$ C,-0.7235541698,-3.2445637173,-1.1592854411 $\mathrm{H},-1.2852836231,-1.1864044756,-1.4688363872$ H,1.2013145931,-1.9808207363,1.959491099 H,1.1837016941,-4.3922411858,1.4095788206 $\mathrm{H},-1.2625820036,-3.5955315799,-2.0307040681$ H,-0.0368090289,-5.2013115942,-0.5945825731

State $=1-\mathrm{A}$ NImag $=0$

\section{N,N-dimethylaniline (DMA)H+ dma+.log.acs}

Brutoformula: C8H12N1(1+)

1,1

$\mathrm{N}, 0.0233320598,0.1311761299,-1.6154782852$

C, $-0.0317866445,0.1369475811,-0.1238886628$ C, $-0.1484788214,0.2037468244,2.6324470909$ C, $0.2495779566,-1.0241386756,0.5866216019$ C, $-0.3690574469,1.3271003616,0.5077739022$ C, $-0.4265711733,1.3553770544,1.9004263826$ C, $0.1881363374,-0.9807942139,1.9777137339$ $\mathrm{H}, 0.5121793825,-1.9500235688,0.0889237866$ $\mathrm{H},-0.5859931293,2.2253838591,-0.0628391329$ $\mathrm{H},-0.6882249772,2.2765742453,2.4059284018$ $\mathrm{H}, 0.4042731564,-1.8759983101,2.5474690659$ H,- $0.1936926854,0.2277425568,3.7143059601$ C, $1.4092778054,-0.1355177461,-2.1551743713$ $\mathrm{H},-0.2183331379,1.0831685171,-1.8994426356$ C,-1.0102120477,-0.7756593715,-2.2420809093 H,1.6954047859,-1.1516061184,-1.8945971279 H,2.0974534424,0.5749188295,-1.7018835486 H, 1.3892958222,-0.0165954503,-3.2377681394 $\mathrm{H},-0.7738148215,-1.8049188358,-1.9833350338$ H,-0.9790782267,-0.6431779896,-3.3228363951 H,-1.9881098237,-0.5060715304,-1.8486098127 
$\mathrm{HF}=-366.6836647 \mathrm{NImag}=0$

\section{4-NH2-DMA}

4nh2dma.log.acs

\section{Brutoformula: C8H12N2}

0,1

H,-2.0392472539,0.0638699323,2.1622600238

C,-1.1317764615,0.0680935774,1.5659668095

C, $1.1569573858,0.0834778699,0.0074497096$

C,-1.2300567321,0.0897412313,0.1825739158

C, $0.1162458053,0.0668403746,2.2053422926$

C, $1.2552816871,0.0801440254,1.3989549912$

C, $-0.0878207074,0.0910268336,-0.63817879$

H,-2.2152093392,0.1297659548,-0.265985113

$\mathrm{N}, 0.210556158,-0.0093939111,3.6065439662$

$\mathrm{H}, 2.2391388291,0.0731969392,1.8583906856$

$\mathrm{H}, 1.0729908253,0.350462753,3.9902539629$

H,2.0730149992,0.0787994311,-0.5680729114

$\mathrm{H},-0.5882735883,0.359970367,4.1027373133$

$\mathrm{N},-0.2223887906,0.1512192514,-2.0486979097$ C, $0.9930392189,0.3399235622,-2.8212240036$ C,-1.1218077882,-0.823050683,-2.6619605195 $\mathrm{H},-0.6944616444,-1.8403660197,-2.669540038$ $\mathrm{H},-1.3234607046,-0.5268356368,-3.6942285873$ H,-2.0739380367,-0.8612486029,-2.1361070941 H,1.6667979839,-0.5349685784,-2.8021114924 $\mathrm{H}, 1.5437766404,1.2079183646,-2.454189903$ $\mathrm{H}, 0.7213252702,0.5294769659,-3.8618756757$

$H F=-421.6860698$ NImag $=0$

\section{Proton Sponge}

me2nnapth.log.acs

\section{Brutoformula: C14H18N2}

0,1 H,0.0381618202,-1.8754354489,-2.852875459 C,-0.0336947752,-1.8154940902,-1.7755285969 $\mathrm{N},-0.0198902562,0.6058769098,-1.9398274973$ C,-0.0519412603,-0.5715021014,-1.1590318791 C,- $-0.4314667491,-2.966407418,0.2964965822$ C, $-0.1252340519,-0.5045317537,0.2883367293$ C,-0.1871241261,-3.0115492787,-1.0497341822 C, $-0.4283425686,-1.725668251,0.9862085701$ C, $0.0798291134,0.683854191,1.0948233653$ H,-0.1705074716,-3.9591665043,-1.5769505431 $\mathrm{H},-0.9890579648,-2.63813902,2.8617309778$ $\mathrm{H},-0.6270063359,-3.8725163752,0.8590677008$ C,-0.2556647475,0.6497483107,2.4417449902 $\mathrm{N}, 0.6312339633,1.8570499698,0.5322802501$ $\mathrm{H},-0.1224007259,1.5360612758,3.0468256681$ C,-0.6919034877,-0.5297985961,3.0735922569 $\mathrm{H},-0.9381726556,-0.5073848877,4.1295566185$ C,- $0.7282364615,-1.7057012114,2.3735841637$ C,-1.1850501924,1.4830775892,-1.8284220614 C, $0.4433902334,0.5006391335,-3.3125295656$ C,0.4519822364,3.106557436,1.2510393754 C, $1.9838942483,1.7352339347,-0.010822767$

\section{4-NH2-DMA H+}

4nh2dma+.log.acs

Brutoformula: C8H13N2(1+)

1,1

$\mathrm{H},-1.8609829205,0 ., 2.1788455424$

C, $-0.9610812838,0 ., 1.575123932$

C, $1.3419587372,0 ., 0.0090454817$

C,-1.0708264232,0.,0.1950251761

C, $0.303402987,0 ., 2.2055834569$

C,1.4545391811,0.,1.3898118579

C, $0.0835108788,0 .,-0.5843123585$

H,-2.0589990115,0.,-0.2506152884

$\mathrm{N}, 0.4060120771,0 ., 3.5627811347$

$\mathrm{H}, 2.4380835842,0 ., 1.8444295869$

H,1.30193282,0.,4.0198216457

H,2.2455850397,0.,-0.5942307653

$\mathrm{H},-0.4108975255,0 ., 4.1493931644$

$\mathrm{N},-0.0021322409,0 .,-2.0705774185$

H,0.9670258609,0.,-2.3954833462

C,- $0.6372547538,1.2497323339,-2.6321985001$

C,-0.6372547538,-1.2497323339,-2.6321985001

H,- $0.1080165395,-2.1121428374,-2.2327985506$ $\mathrm{H},-0.5676561451,-1.2222339415,-3.7191842884$ $\mathrm{H},-1.6797643735,-1.2777195081,-2.3244520753$ H,-0.1080165395,2.1121428374,-2.2327985506 H,-1.6797643735,1.2777195081,-2.3244520753 H,-0.5676561451,1.2222339415,-3.7191842884

State $=1-A^{\prime}$ NImag $=1$

\section{Proton Sponge $\mathbf{H}+$}

me2nnapth+.log.acs

Brutoformula: C14H19N2(1+)

1,1

H,-2.65497407,-0.0907591075,-2.1230468862

C,-2.2906898358,-0.0192426134,-1.1048493945

$\mathrm{N}, 0.0129509789,-0.1606505157,-1.9725582322$

C, $-0.935850383,-0.0414384528,-0.864804174$

C,-2.7819839272,0.1864877005,1.2417532468

C, $-0.4380325147,0.0521013937,0.4753731796$

C,-3.2225079131,0.0948620703,-0.0532922871

C, $-1.3964995887,0.1681975977,1.541847833$

C, $0.9378404275,0.0409045344,0.858942849$

$\mathrm{H},-4.2820884819,0.1092783714,-0.2770467796$

$\mathrm{H},-1.6955626126,0.3501142821,3.6697265866$

H,-3.4886404464,0.2745963228,2.0587265852

C, $1.3383106582,0.1347609931,2.1683133818$

N, $1.9627553064,-0.0770606225,-0.2067598433$

$\mathrm{H}, 2.3915179519,0.1230522904,2.4238624783$

C,0.3829952528,0.2477284774,3.1981760673

$\mathrm{H}, 0.7120336449,0.3210168522,4.2269807432$

C,- $0.9523609819,0.263563968,2.8855297149$

C,-0.1203493683,-1.443229407,-2.7036042962

C, $-0.0454824521,0.9928485053,-2.9016588828$

C, $2.8380222161,1.1325544792,-0.3193278223$

H, $1.332473733,-0.1308614851,-1.1079323667$ 
H,1.3853011552,-0.0491207281,-3.3556702952 $\mathrm{H}, 0.6191461441,1.5090066178,-3.6966898584$ H, $-0.2790103866,0.0110644136,-3.9886559583$ $\mathrm{H},-0.9126031694,2.4992023455,-2.1269243316$ H,- $1.5445859098,1.5155620828,-0.8043277623$ $\mathrm{H},-2.007084622,1.1367990471,-2.4773067551$ $\mathrm{H}, 2.7417420704,1.8229232104,0.7858440687$ $\mathrm{H}, 2.1600095963,2.5262371618,-0.7450827925$ $\mathrm{H}, 2.1138380622,0.7777901034,-0.5063086124$ H,1.0733638457,3.1891557954,2.1597502028 H,- $0.5941017099,3.2366343286,1.5340262866$ H,0.7309378405,3.9300910562,0.5882796894

State $=1-\mathrm{A}$ NImag $=0$

\section{4-H2N-Pyridine}

py4nh21.log.acs

Brutoformula: C5H6N2

0,1

H,-2.0566794072,-0.0216813539,1.981989815

$\mathrm{N}, 0 .,-0.0250750284,2.1146395156$

C, $1.1351466172,-0.0057752278,1.4061275201$

C, $-1.1351466172,-0.0057752277,1.40612752$

$\mathrm{H}, 2.0566794071,-0.0216813541,1.9819898152$

C, $1.1960369637,0.0308601596,0.0193167206$

H, 2.1566239623, $0.0483203003,-0.4846423485$

C, $0.0000000001,0.0491668859,-0.7142175655$

$\mathrm{N}, 0.0000000002,0.1324266994,-2.0945235331$

$\mathrm{H},-2.1566239621,0.0483203001,-0.4846423486$

C, $-1.1960369635,0.0308601597,0.0193167205$

$\mathrm{H}, 0.8458677138,-0.1304560141,-2.5756534498$

$\mathrm{H},-0.8458677144,-0.130456012,-2.5756534494$

$\mathrm{HF}=-303.7324008$ NImag $=0$

\section{3-H2N-Pyridine}

py3nh21.log.acs

\section{Brutoformula: C5H6N2}

0,1

H,-1.6117507454,0.0697830168,1.7014723212 $\mathrm{N}, 0.4448187059,0.0764467774,1.821005171$

$\mathrm{C}, 1.6002886689,0.0661703615,1.1485030641$

C, $-0.6884346555,0.0580171122,1.1248479747$

$\mathrm{H}, 2.5080817228,0.0810395075,1.7430593267$

C, $1.6547486153,0.0362485444,-0.2434365013$

$\mathrm{H}, 2.6115486442,0.0280053931,-0.752797485$

C, $0.4698486221,0.0179017614,-0.968663053$

$\mathrm{H}, 0.4838925201,0.0008513089,-2.0539406524$

$\mathrm{N},-1.9715295622,0.0672018981,-0.945766219$

C, $-0.7481801865,0.0274309194,-0.2793711635$

$\mathrm{H},-1.9783215039,-0.3023975154,-1.8847918061$

H,-2.7750108457,-0.2266990853,-0.4101209792

$\mathrm{HF}=-303.7275259 \mathrm{NImag}=0$
C, $2.7623174302,-1.3399624571,-0.1186108363$ H,-0.9910958797, 1.0291969963,-3.4531315951 $\mathrm{H}, 0.7707955235,0.9119367166,-3.6223728207$ $\mathrm{H}, 0.0630948728,1.9227681367,-2.3423427628$ H,-0.065990062,-2.2755521719,-2.0009951012 $\mathrm{H}, 0.6958003421,-1.5296497461,-3.4238228923$ H,-1.0691199302,-1.5103069408,-3.2467089131 H,3.3936743134,-1.4169370191,-1.00395245 H,2.0793394063,-2.1861602621,-0.0723381278 H,3.3849220869,-1.3258356247,0.774850482

H,3.4687302908,1.0272158189,-1.2022111357 H,3.4627726386, 1.2249767479,0.5679490762 H,2.2079985617,2.0150670532,-0.4136990668

State $=1-\mathrm{A}$ NImag $=0$

4-H2N-Pyridine $\mathrm{H}+$

py 4 nh2+1.log.acs

Brutoformula: C5H7N2(1+)

1,1

H,0.,-0.0000000012,-2.8478065961

$\mathrm{N}, 0 .,-0.0000000001,-1.8352580786$

C, $0 ., 0.000000002,0.9309344066$

C,1.1844891946,0.0000000002,-1.1710682185

C,-1.1844891946,0.0000000009,-1.1710682185

C,-1.2159934411,0.000000001,0.1933094446

C, $1.2159934411,0.0000000012,0.1933094445$

H,2.0802330567,-0.0000000009,-1.7772231923

$\mathrm{H},-2.0802330567,-0.0000000002,-1.7772231922$

$\mathrm{H},-2.1723717189,-0.0000000007,0.6999155175$

$\mathrm{H}, 2.1723717189,0.0000000002,0.6999155176$

$\mathrm{N}, 0 ., 0.0000000002,2.2691471895$

$\mathrm{H},-0.8599474071,-0.0000000013,2.7965579588$

H,0.8599474071,-0.0000000012,2.7965579589

$\mathrm{HF}=-304.1214467 \mathrm{NImag}=0$

\section{3-H2N-Pyridine $\mathbf{H}^{+}$}

py 3 nh $2+1 . \log$.acs

Brutoformula: C5H7N2(1+)

1,1

H,-1.9867080093,0.0000000039,1.7036511748

N,-1.1142603914,0.000000001,1.1837441223

C, $1.2033148471,-0.0000000005,-0.2213603389$

C,-1.1952379846,-0.0000000039,-0.1611129991

C, $0.0377113263,-0.000000002,1.8778457619$

C, $1.226515126,-0.0000000037,1.1616206442$

C, $-0.0223769472,-0.0000000026,-0.9310222186$

$\mathrm{H},-2.1883258429,-0.0000000006,-0.5885411282$

$\mathrm{H},-0.0305606389,0.000000001,2.9556633922$

H,2.1668925093,0.0000000038,1.6965442721

$\mathrm{N},-0.0688421163,0.0000000006,-2.2834510226$

H,2.1360914425,-0.0000000013,-0.7740206611

$\mathrm{H}, 0.7760522849,0.0000000041,-2.8314494748$

$\mathrm{H},-0.9402655613,0.0000000003,-2.7881111522$

$H F=-304.1056998$ NImag $=0$ 


\section{2-H2N-Pyridine}

py2nh2.log.acs

Brutoformula: C5H6N2

0,1

N,1.9001413012,-0.0074925011,1.3324106254

$\mathrm{N},-0.410450678,-0.0260353244,1.2940348925$

C, $-1.5625107174,0.0272581855,0.6175990214$

C, $0.7306074619,-0.0068643453,0.5949758766$

$\mathrm{H},-2.4633939958,0.0124361341,1.2250549296$

C,-1.6484915682,0.0921875215,-0.7676059595

$\mathrm{H},-2.6110244584,0.1296683481,-1.2614886466$

C,-0.4519269011,0.1052848671,-1.4896420873

$\mathrm{H},-0.4624481851,0.1542972084,-2.5730490969$

H, $1.6979583031,0.079125964,-1.3455631509$

C, $0.7541479023,0.0588159742,-0.8123884009$

H, 1.7887323431,-0.2748209396,2.2991844226

H,2.7386591924,-0.3438610913,0.8864775738

$\mathrm{HF}=-303.7382815$ NImag $=0$

\section{2,6-Me2-Pyridine}

i26me1.log.acs

Brutoformula: C7H9N1

0,1

C,-2.4194996096,-0.0055701865,1.0126131487

N,-0.002911269,-0.0233758677,0.8671076547

C, $1.1566685101,-0.0159188428,0.1957025413$

C,-1.1578980014,0.0054313891,0.188424045

C, $2.4123868042,-0.0480095353,1.0282650081$

C, $1.2025373924,0.0208658185,-1.2017591387$

$\mathrm{H}, 2.1556529658,0.0258459827,-1.7174031727$

C, $0.0065449505,0.0506895248,-1.9103314822$

$\mathrm{H}, 0.0101829787,0.0793482549,-2.9946451492$

$\mathrm{H},-2.1437637785,0.0663627638,-1.7310147529$

C,-1.1941997611,0.0433077992,-1.2093084541

H,-2.4298963336,0.8461841711,1.6978723729

H,-2.4632045981,-0.9106722996,1.6244934763

H,-3.3126206935,0.0369724875,0.3864065699

H,3.3100800323,-0.050399856,0.4072517426

H,2.4218598568,-0.9378654317,1.6633182529

H,2.4474918469,0.8201462341,1.6920564752

$\mathrm{HF}=-327.0134325 \mathrm{NImag}=0$

\section{4-MeO-Pyridine}

i4meopy1.log.acs

\section{Brutoformula: C6H7N1O1}

0,1

H, 2.2277354789,0.0000000082, 1.97369759

$\mathrm{N}, 0.2591653217,0.0000000023,2.5726562621$

C,-1.0137028588,-0.0000000047,2.1493659477

C, $1.1977682479,0.0000000029,1.6266907125$

H,- $1.7743391598,0.0000000085,2.9248809843$

C,-1.3918678681,-0.0000000044,0.8156952487

H,-2.4340232775,0.0000000012,0.5217115893

\section{2-H2N-Pyridine $\mathbf{H}+$}

py2nh2+.log.acs

Brutoformula: C5H7N2(1+)

1,1

H, $0.4627473758,0.0404565062,-2.0889161928$

$\mathrm{N}, 0.4253279787,0.0128962371,-1.0760277984$

C, $0.3842523113,-0.0656728123,1.6406852916$

C, $-0.7878145972,0.0484503225,-0.4617315408$

C, $1.6135007092,-0.0594241435,-0.4002318375$

C,1.6223839321,-0.100017317,0.9621990669

C, $-0.8015023388,0.0070424337,0.9485266852$

$\mathrm{N},-1.9005048709,0.120193182,-1.2091260547$

$\mathrm{H}, 2.5019662591,-0.0805730519,-1.0159906743$

H, 2.5597832113,-0.1572099242,1.4968889601

$\mathrm{H},-1.753246396,0.0339077237,1.4629439608$

$\mathrm{H}, 0.3650832865,-0.0968909856,2.723607244$

$\mathrm{H},-1.8864525994,0.1496225156,-2.2171281565$

H,-2.8055242627,0.1472193135,-0.7656989535

$\mathrm{HF}=-304.1132561 \mathrm{NImag}=0$

\section{2,6-Me2-Pyridine $\mathrm{H}+$}

i26me+21.log.acs

Brutoformula: C7H10N1(1+)

1,1

H,0.0000622607,0.072434992,-1.7139885564

N,0.0000609986,0.0301969078,-0.6984871388

C, $0.0001155393,-0.081699755,1.9982162148$

C,-1.2094946656,0.0308033105,-0.0755479064

C, $1.2096997788,-0.0224647961,-0.0777681178$

C,1.210178262,-0.0815461974,1.3069310286

C,-1.2099121031,-0.0250050876,1.3093606875

C, $-2.4350162244,0.0771726351,-0.9319053249$

C,2.4349298603,-0.0012393084,-0.9355773219

$\mathrm{H}, 2.1538006761,-0.1255511238,1.8337935394$

H,-2.1535290209,-0.0247278291,1.8380799967 $\mathrm{H}, 0.0001538523,-0.1262074065,3.0811259418$

$\mathrm{H},-3.3245715409,0.2049103444,-0.3176362957$

H,-2.5455193367,-0.8528942155,-1.4987788491

H,-2.3893039928,0.9052457012,-1.644975915

$\mathrm{H}, 2.5301945582,0.9596173743,-1.4513511507$

H,2.40107195,-0.7903450146,-1.6923460031

H,3.327008537,-0.1487074273,-0.3294866471

$\mathrm{HF}=-327.3942812$ NImag $=0$

\section{4-MeO-Pyridine $\mathrm{H}^{+}$}

i4meopy+1.log.acs

Brutoformula: C6H8N1O1(1+)

1,1

$\mathrm{H}, 0.3792838898,0.0000000076,-3.2945671938$

$\mathrm{N}, 0.1685846377,0.0000000024,-2.3030476043$

C,-0.4002463064,-0.0000000085,0.3851538798

C, $1.1880463134,-0.0000000018,-1.4157009723$

C,-1.1312555463,-0.0000000008,-1.9052730701

C, $-1.4406774641,-0.0000000047,-0.5748137592$

C, $0.9393151648,-0.0000000056,-0.0656411331$ 
C, $-0.3917771352,-0.0000000121,-0.163383372$ $\mathrm{O},-0.8041475164,-0.0000000021,-1.4541297734$ $\mathrm{H}, 1.7662639466,-0.0000000025,-0.4442843487$ C,0.9410996988,-0.0000000064,0.2545438593 C, $0.1790446607,0.0000000013,-2.4858662985$ $\mathrm{H}, 0.8073365969,0.8948712631,-2.4347924375$ $\mathrm{H},-0.3758926887,0.0000000102,-3.4219935502$ H,0.8073365909,-0.8948712655,-2.4347924497

$H F=-362.9108961$ NImag $=0$

\section{2-MeO-Pyridine \\ meo2py.log.acs}

\section{Brutoformula: C6H7N1O1}

0,1

O,-0.7158883631,0.0000000012,-1.6809944535 $\mathrm{N}, 0.9504981048,0.0000000067,-0.0768716144$ C, $1.2809502207,0.0000000066,1.2249511165$ C, $-0.3381393937,0.0000000107,-0.3835043822$ $\mathrm{H}, 2.345491944,-0.000000003,1.4402597858$ C, $0.3511427536,-0.0000000024,2.2523505167$ $\mathrm{H}, 0.6723075864,-0.0000000117,3.2862636601$ C,-1.0064343928,0.0000000013,1.9073387902 $\mathrm{H},-1.7703537516,-0.0000000042,2.6769579224$ $\mathrm{H},-2.4012099654,0.0000000012,0.25047688$ C, $-1.3683522336,0.000000005,0.5735839255$ C, $0.3164069391,-0.0000000021,-2.6744655361$ $\mathrm{H}, 0.9454842422,0.8877504419,-2.5838138817$ H,-0.2073880664,-0.000000011,-3.6287187532 $\mathrm{H}, 0.9454842486,-0.8877504403,-2.5838138691$

$\mathrm{HF}=-362.9209596 \mathrm{NImag}=0$

\section{2-Me-Pyridine}

py2me1.log.acs

\section{Brutoformula: $\mathrm{C} 6 \mathrm{H} 7 \mathrm{~N} 1$}

0,1 C,-0.1946586495,-0.0061360372,2.2206890871 $\mathrm{N},-1.3074633147,-0.0524110237,0.0689688923$ C,-1.2977708411,-0.0535821756,-1.2656287638 C,-0.1324276026,-0.0045976759,0.71570889 $\mathrm{H},-2.2709763592,-0.0930708692,-1.7479498174$ C,-0.1344067325,-0.0079410034,-2.0313453916 $\mathrm{H},-0.1879428783,-0.0112426178,-3.1134276505$ C, $1.0843648214,0.0417409048,-1.3625191886$ H,2.0180507508,0.0787728794,-1.9130533195 H,2.0182998791,0.0819386613,0.5806127707 C, $1.0859841193,0.0435165357,0.0289446689$ $\mathrm{H}, 0.7998550282,0.0435203613,2.6677567103$ $\mathrm{H},-0.6946244005,-0.9110465971,2.5765333078$ H,-0.7820444843,0.8452069574,2.5748171171

$\mathrm{HF}=-287.6823928 \mathrm{NImag}=0$
$\mathrm{H}, 2.1891891375,0.0000000035,-1.8256861928$ $\mathrm{H},-1.8786423538,0.0000000032,-2.6869572151$ $\mathrm{H},-2.4713007838,-0.0000000023,-0.2463548437$ $\mathrm{H}, 1.7741540897,0.0000000003,0.6195979719$ $\mathrm{O},-0.7734762229,-0.0000000009,1.6448657324$ C, $0.2038512157,0.0000000011,2.7139799228$ $\mathrm{H}, 0.8176177366,-0.9010029958,2.6631192979$ H,- $0.3820613884,0.0000000111,3.6282061133$ $\mathrm{H}, 0.8176177455,0.9010029913,2.6631192854$

$\mathrm{HF}=-363.2913352 \mathrm{NImag}=0$

\section{2-MeO-Pyridine $\mathrm{H}+$}

meo2py+.log.acs

Brutoformula: C6H8N1O1(1+)

1,1

H,-2.2958754335,-0.0000000026,0.187262433

$\mathrm{N},-1.3457890522,0.0000000034,0.5496388334$

C, $1.2113126045,0.0000000067,1.4507961968$

C, $-0.3465750208,0.0000000087,-0.370722087$

C,-1.1317915413,0.0000000007,1.8899681157

C,0.1520181097,0.0000000006,2.3720508519

C, $0.9754919663,0.0000000049,0.0853001085$

O,-0.7835711824,0.000000006,-1.6081832226

$\mathrm{H},-2.0154303812,-0.0000000075,2.513585094$

$\mathrm{H}, 0.3287042563,-0.0000000059,3.4383818397$

H, 1.7919281037,-0.000000007,-0.6219368294

H,2.2340792972,0.0000000006,1.8092693373

C, $0.1559269572,-0.0000000008,-2.7175798631$

$\mathrm{H}, 0.7685773289,0.9017047967,-2.6858010342$

H,- $0.4675833076,-0.0000000029,-3.6062288345$

$\mathrm{H}, 0.7685773232,-0.9017048018,-2.6858010276$

$\mathrm{HF}=-363.29038 \mathrm{NImag}=0$

2-Me-Pyridine $\mathrm{H}+$

py 2 me+ $+\log$.acs

Brutoformula: C6H8N1(1+)

1,1

H, $0.5844331866,0.0000000014,-1.9764171436$

$\mathrm{N}, 0.5770363961,0.000000002,-0.960629641$

C, $0.5799473688,-0.0000000053,1.749349455$

C, $-0.627553079,-0.0000000005,-0.3397277189$

C, $1.7702801435,-0.0000000095,-0.3174026714$

C, $1.7966037507,-0.0000000055,1.0573614285$

C, $-0.6225072431,-0.0000000041,1.053215389$

C,-1.8684692846,0.0000000006,-1.1742049088

$\mathrm{H}, 2.6545680194,-0.0000000009,-0.9402002428$

$\mathrm{H}, 2.7453850773,0.00000001,1.5764856542$

$\mathrm{H},-1.5687203981,0.0000000012,1.5789446856$

$\mathrm{H}, 0.574597342,0.0000000073,2.8330090484$

$\mathrm{H},-1.6475234562,0.0000000058,-2.2426825816$

$\mathrm{H},-2.4740389135,0.8815416535,-0.9485503718$

$\mathrm{H},-2.4740389111,-0.881541656,-0.9485503796$

$\mathrm{HF}=-288.0569505 \mathrm{NImag}=1$

Pyridine H+

\section{Pyridine}


py1.log.acs

Brutoformula: C5H5N1

0,1

$\mathrm{H},-2.0569337124,0.0000000058,1.4964644292$

$\mathrm{N}, 0 .,-0.000000007,1.6051007526$

C, $1.1419667562,0.0000000025,0.9105067751$

C,-1.1419667562,-0.0000000052,0.9105067751

$\mathrm{H}, 2.0569337123,0.0000000059,1.4964644292$

C, $1.1967520894,-0.0000000065,-0.4824393935$

$\mathrm{H}, 2.1537963074,0.0000000034,-0.990868437$

C, $0 .,-0.0000000048,-1.1941613769$

$\mathrm{H},-0.0000000001,0.0000000023,-2.2782661223$

$\mathrm{H},-2.1537963073,0.0000000046,-0.9908684371$

C,-1.1967520894,-0.0000000011,-0.4824393934

$\mathrm{HF}=-248.3512161 \mathrm{NImag}=0$

\section{3-Cl-Pyridine}

py3cl.log.acs

Brutoformula: C5H4C11N1

0,1

$\mathrm{H},-2.0689558583,0.000000001,1.4896703709$

$\mathrm{N},-0.0028044006,0.0000000014,1.5999590151$

C, $1.1414417569,-0.0000000047,0.9103563222$

C, $-1.1477791408,-0.000000001,0.9163400893$

H,2.0544606092,0.0000000019, 1.4976860341

C, $1.1945278371,-0.0000000006,-0.4819402999$

$\mathrm{H}, 2.1483815026,0.0000000029,-0.9959828288$

C, $0.0019295027,-0.0000000006,-1.1990908731$

$\mathrm{H},-0.0096049791,-0.000000001,-2.2817183994$

$\mathrm{Cl},-2.7307428154,0.0000000016,-1.3066245175$

C,-1.1852433033,-0.0000000009,-0.477390206

$\mathrm{HF}=-707.9725709 \mathrm{NImag}=0$

\section{2-Cl-Pyridine}

py2cl.log.acs

Brutoformula: C5H4C11N1

0,1

$\mathrm{Cl},-2.6222616873,-0.0000000029,1.8398750904$ N,-0.0019228282,0.0000000078,1.6007764012 C, $1.1458490588,0.0000000158,0.9095236419$

C,-1.1228817722,0.0000000035,0.9117644679

H,2.0548984882,-0.0000000214, 1.5026529066

C, $1.1987871222,0.0000000101,-0.4797305596$

H, $2.1529448115,-0.0000000106,-0.9918296607$

C, $-0.0042836385,0.0000000141,-1.1850206733$

$\mathrm{H},-0.0118811823,-0.0000000015,-2.2690630582$

$\mathrm{H},-2.1612259092,-0.0000000155,-0.982292059$

C,-1.202467966,0.0000000005,-0.4819455921

$\mathrm{HF}=-707.976559 \mathrm{NImag}=0$ py.log.acs

Brutoformula: C5H6N1(1+)

1,1

H, $0 .,-0.0000000008,-2.3469592894$

N, $0 ., 0.0000000005,-1.3308007819$

C,0.,0.0000000014,1.3894636253

C,-1.1881546124,0.,-0.6887238748

C, $1.1881546124,0.0000000015,-0.6887238748$

C, $1.2097851713,0.0000000011,0.692136098$

C, $-1.2097851713,0.0000000008,0.692136098$

$\mathrm{H},-2.0768852418,-0.0000000008,-1.3051836278$

$\mathrm{H}, 2.0768852418,-0.0000000003,-1.3051836278$

$\mathrm{H}, 2.1597062303,-0.0000000022,1.2094466709$

H,-2.1597062304,-0.0000000006, 1.2094466709

H,0.,-0.0000000006,2.4729459124

$\mathrm{HF}=-248.7190677 \mathrm{NImag}=0$

\section{3-Cl-Pyridin $\mathrm{H}+$}

py $3 \mathrm{cl}+. \log$.acs

Brutoformula: C5H5C11N1(1+)

1,1

H,-0.0068078188,0.0000000033,-2.3433930054

$\mathrm{N},-0.0016076268,-0.0000000019,-1.3267004858$

C, $0.0022245323,-0.0000000055,1.3945763261$

C,-1.1911864433,-0.0000000012,-0.6903610717

C, $1.1880188826,-0.000000005,-0.6904801466$

C, $1.2048408856,-0.0000000033,0.6919591688$

C,-1.2110148562,-0.0000000042,0.6965669865

$\mathrm{H},-2.0843299605,0.0000000028,-1.2998848924$

$\mathrm{H}, 2.0754704821,0.0000000013,-1.3081776575$

$\mathrm{H}, 2.1528224568,0.0000000084,1.2135091554$

$\mathrm{Cl},-2.7208467472,0.0000000043,1.5212020238$

$\mathrm{H},-0.0028559275,0.000000001,2.4779443747$

$\mathrm{HF}=-708.3308056 \mathrm{NImag}=0$

2-Cl-Pyridine $\mathbf{H}+$

py $2 \mathrm{cl}+. \log$.acs

Brutoformula: C5H5C11N1(1+)

1,1

$\mathrm{H},-0.0076303683,0.000000004,-2.353371085$

$\mathrm{N},-0.0008649556,-0.0000000026,-1.3361131545$

C, $-0.0052810451,-0.0000000056,1.3822551445$

C,-1.1929420773,-0.0000000036,-0.6937275718

C, $1.1887936212,-0.0000000074,-0.686656764$

C, $1.2103378421,-0.0000000027,0.6894264049$

C,-1.2143370768,-0.0000000067,0.6929886065

Cl,-2.602309153,0.0000000084,-1.6570023828

$\mathrm{H}, 2.0739849245,-0.0000000007,-1.3084415894$

H,2.158470144,0.0000000119,1.2096584759

$\mathrm{H},-2.1648455548,0.0000000012,1.208712321$

H,-0.0115811299,0.0000000039,2.4659690216

$H F=-708.332296$ NImag $=0$

Et3P H+

\section{Et3P}


et3p1.log.acs

Brutoformula: C6H15P1

0,1

C, $0.5792564698,1.638026311,0.3914936518$

$\mathrm{P},-0.6549167732,0.2885913466,-0.0262494119$

C, $-0.4494312426,0.3171704676,-1.8901209859$

C, $0.3322092494,-1.2607524459,0.3719984931$

$\mathrm{H}, 1.3995786571,-1.0639934986,0.2152650556$

$\mathrm{H}, 0.0402378148,-2.0088366376,-0.3717818787$

C, $0.0815626023,-1.8265340978,1.7756971468$

H,-0.653477884,1.345927063,-2.2048295899

C, $-1.392416586,-0.6408197028,-2.6273029559$

$\mathrm{H}, 0.5945602608,0.1076544152,-2.1521876098$

C, $0.7725095026,1.8824248113,1.8927531034$

$\mathrm{H}, 0.1935414254,2.5491986574,-0.0785248831$

$\mathrm{H}, 1.5414348923,1.4176162513,-0.0868402972$

$\mathrm{H},-1.2624680667,-0.5594440345,-3.7105377567$

$\mathrm{H},-2.4382794818,-0.4208502416,-2.3970181996$

H,-1.2072349401,-1.6842255394,-2.3563257201

H,-0.1822593807,2.0726450459,2.3908591086

$\mathrm{H}, 1.417896363,2.7488845796,2.0648374544$

$\mathrm{H}, 1.2401506006,1.0265732245,2.3856907373$

$\mathrm{H}, 0.6509030868,-2.7480929733,1.9314023785$

$\mathrm{H},-0.9768529383,-2.0574410578,1.9219789022$

$\mathrm{H}, 0.3734963685,-1.1237219429,2.5597432557$

$\mathrm{HF}=-579.1295367 \mathrm{NImag}=0$

\section{MePh2P}

meph2p.log.acs

\section{Brutoformula: C13H13P1}

0,1

C, $-2.7408872314,0.0000001367,-0.2809444871$

$\mathrm{P},-1.1415113476,-0.0000000469,-1.2291536164$

C, $-0.2424105178,1.4453752294,-0.475206307$

C, $-0.2424106675,-1.4453753183,-0.4752061269$

C, $1.2033223037,3.6596807116,0.483751112$

C, $-0.3597236847,1.8548111713,0.8594912287$

C, $0.6069295346,2.1710765597,-1.3206095973$

C, $1.3290653505,3.2656211019,-0.8461311918$

C, $0.3542589986,2.9526445205,1.3348292106$

H,-1.0101457417,1.3208710989,1.5425347686 $\mathrm{H}, 0.6978845184,1.8779822933,-2.3613798812$ H,1.9825222358,3.8125401667,-1.5169621097 $\mathrm{H}, 0.2489277597,3.25566138,2.3710240439$

H,1.7588119672,4.5136371732,0.8551385906

C, $1.2033221105,-3.6596807195,0.4837515633$

C, $0.6069303572,-2.1710759944,-1.3206090075$

C, $-0.359724853,-1.8548119038,0.8594911225$

C, $0.3542578071,-2.9526452165,1.3348292322$

C, $1.3290661638,-3.2656204768,-0.84613046$

H,0.6978861215,-1.8779812561,-2.3613790911

H,-1.0101477346,-1.3208724108,1.5425343291

$\mathrm{H}, 0.2489257581,-3.2556625911,2.3710238265$

H,1.982523831,-3.8125390103,-1.5169610575

$\mathrm{H}, 1.7588117514,-4.5136371487,0.8551391536$

H,-2.6353307557,0.0000003377,0.8057516585 et $3 p+. \log . a c s$

Brutoformula: C6H16P1(1+)

1,1

C, $-0.5538788383,-1.6557626385,-0.3236295518$

P, $0.0007405913,-0.0025328654,0.2168711117$

$\mathrm{H}, 0.0008174892,-0.0060480655,1.6241363794$

C, $-1.1524725167,1.3090384714,-0.3159299412$

C, $1.7115845607,0.3455372139,-0.3187067953$

$\mathrm{H},-1.5838059156,-1.7651071183,0.0276245306$

C,0.3214785674,-2.8133486451,0.1820547721

$\mathrm{H},-0.5914686405,-1.6326707755,-1.41756553$

C,-2.5945267521,1.125251212,0.1821651013

$\mathrm{H},-1.1093478969,1.3407222948,-1.4095025562$

H,-0.7325240098,2.2518386067,0.045825998

$\mathrm{H}, 2.3227753631,-0.485827903,0.0434753401$

C,2.2679428946,1.6899451569,0.1761888041

H, 1.7161803146,0.2912117537,-1.4122661528

H, $0.3775235201,-2.8357115497,1.272764405$

H, $1.3352735181,-2.7703225263,-0.2193751136$

$\mathrm{H},-0.1180582208,-3.7575567024,-0.1430862693$

H,-2.6470375989,1.0824174806,1.2724096038

$\mathrm{H},-3.0603193309,0.226690982,-0.2257882605$

$\mathrm{H},-3.1925443865,1.9784248264,-0.1417144885$

H,2.2603448221,1.758321694,1.2663771674

$\mathrm{H}, 1.7169962391,2.5392434787,-0.2312661574$

H,3.3043262246,1.7862456187,-0.1510623967

$H F=-579.5107768$ NImag=0

\section{MePh2P H+}

meph2p+1.log.acs

Brutoformula: C13H14P1(1+)

1,1

C, $0.536898288,0.2304951311,2.662913475$

$\mathrm{P},-0.463086622,-0.0076921516,1.166170846$

H,-1.7935080972,-0.1186132806,1.6101215935

C,- $-0.0004779793,-1.5106619709,0.2895799072$

C,-0.4446991727,1.4450691349,0.1070525022

C, $-0.3825579437,3.7516297309,-1.4426514892$

C, $0.6443735542,1.6810878689,-0.7448103537$

C,-1.5041687225,2.3607335983,0.1789526294

C,-1.4669589039,3.5137903862,-0.5998410223

C,0.669718121,2.8378453012,-1.5160113439

H, 1.4585857193,0.9685088612,-0.8170637141

$\mathrm{H},-2.3544217599,2.1803621297,0.8278689133$

H,-2.2858684996,4.2210711373,-0.5513216709

H, 1.5073440104,3.0241336361,-2.1770630738

$\mathrm{H},-0.3586861888,4.6494005061,-2.0488676879$

C, $0.6243263818,-3.850635103,-1.0794893739$

C, $-0.6116525713,-1.7820908075,-0.9448745364$

C, $0.9237746299,-2.4118302674,0.8353549349$

C, $1.2341915509,-3.5797094506,0.1434547884$

C,- $0.2974005879,-2.9541275407,-1.6222913846$

H,-1.3210935867,-1.0858573228,-1.3782137617

H,1.4052398409,-2.2171352684,1.7857514513

H, 1.9509965909,-4.2755898189,0.5619033239

$\mathrm{H},-0.7692606619,-3.1671368485,-2.5737190901$

H, $0.8674872648,-4.7619714564,-1.6127525256$ 
$\mathrm{H},-3.3105770489,-0.884522091,-0.5743083008$ $\mathrm{H},-3.3105769869,0.8845223021,-0.5743086058$

$\mathrm{HF}=-844.7180836 \mathrm{NImag}=1$

\section{Ph3P}

ph3p.log.acs

Brutoformula: C18H15P1

0,1

C, $-1.7166287951,-0.000000019,-0.5005226756$

P, $-0.0664469663,-0.000000042,-1.3394562656$

C, $0.8010806207,1.446271485,-0.5554916758$

C, $0.8010806172,-1.4462715435,-0.5554916224$

C, $2.2386847113,-3.6383954506,0.4561796335$

C, $1.5955277021,-2.2333743386,-1.3981445369$

C, $0.7311350029,-1.7814634308,0.8040973528$

C, $1.4433069189,-2.8674770373,1.30535507$

C, $2.3134993161,-3.3198891389,-0.8968526258$

H,1.6501868209,-1.9936615168,-2.4548018254

H,0.1108294991,-1.2003165537,1.4759674644

H,1.3781709512,-3.1137001602,2.3596945143

H,2.9244844729,-3.9170839091,-1.5647129234

H,2.7927295668,-4.4837200727,0.8489987667

C, $2.2386846657,3.638395469,0.4561794771$

C, $0.7311351706,1.7814633001,0.804097325$

C, $1.5955275148,2.2333743899,-1.398144668$

C, $2.3134991032,3.3198892321,-0.8968528091$

C, $1.443307066,2.8674769425,1.3053549923$

H, $0.1108298156,1.2003163336,1.4759674971$

$\mathrm{H}, 1.6501865005,1.9936616225,-2.4548019754$

H,2.9244840988,3.9170840894,-1.564713167

$\mathrm{H}, 1.3781712272,3.1137000086,2.3596944534$

H,2.7927294962,4.4837201236,0.8489985758

C,-4.364902793,0.0000000426,0.4586117935

C,-2.3970240393,-1.2040430843,-0.2648135552

C, $-2.3970238929,1.2040430796,-0.2648133109$

C, $-3.7053604179,1.2032819376,0.2143183925$

C,-3.7053605647,-1.2032818833,0.2143181452

H,-1.9026934229,-2.1506547573,-0.4513183662

$\mathrm{H},-1.902693161,2.150654731,-0.4513179265$

H,-4.2091770885,2.1464194117,0.3962792864

$\mathrm{H},-4.2091773467,-2.1464193361,0.3962788454$

H,-5.3827823692,0.0000000753,0.8318583438

$\mathrm{HF}=-1036.4960772 \mathrm{NImag}=1$

\section{Me2PhP}

phme2p1.log.acs

Brutoformula: C8H11P1

0,1

$\mathrm{H}, 2.1679374405,-0.0000000005,-2.4140060322$

C, $1.1418899676,0.0000000004,-2.0620142804$

C, $-1.484841173,-0.0000000058,-1.1474652797$

C, $0.8780333663,-0.0000000192,-0.6946677114$

C, $0.0893958547,0.0000000122,-2.9782950152$

C,-1.2248294975,0.0000000055,-2.5186083616
$\mathrm{H}, 0.4084725807,-0.6121710137,3.3440516485$ H, $0.2006369644,1.1449274739,3.1545262993$

H,1.5893970841,0.3374296584,2.3963629323

$H F=-845.0987639$ NImag $=0$

Ph3P H+

ph3p+.log.acs

Brutoformula: C18H16P1(1+)

1,1

C, $1.1026381724,0.0000003401,-1.3654534021$

$\mathrm{P}, 0.7072332474,-0.0000000436,0.395538036$

$\mathrm{H}, 1.8949369313,-0.0000001698,1.148927378$

C,- $0.1322757662,-1.5154677453,0.8942001691$

C, $-0.13227586,1.5154673655,0.8942008948$

C,-1.4429346286,-3.8160632246,1.7492201473

C, $-1.3062539118,-1.9300178759,0.2479060774$

C, $0.3834114421,-2.2544712276,1.9677366265$

C, $-0.2755792608,-3.406210878,2.3897953883$

C,-1.9569657863,-3.0798019313,0.680520257

$\mathrm{H},-1.708303226,-1.3717909048,-0.5898049851$

H,1.2913951407,-1.9427331336,2.4718810603

$\mathrm{H}, 0.1245576085,-3.9810276531,3.2160922153$

H,-2.8631834634,-3.4033466959,0.1829752252

$\mathrm{H},-1.9534897653,-4.7125093739,2.0805346743$

C,-1.4429347303,3.8160624055,1.7492220581

C, $0.3834116168,2.2544705781,1.967737411$

C,-1.3062542915,1.9300175416,0.2479073469

C,-1.9569661619,3.0798013787,0.6805221154

C,-0.2755790882,3.4062100091,2.3897967659

$\mathrm{H}, 1.2913955327,1.9427324497,2.4718814336$

$\mathrm{H},-1.7083038488,1.3717907632,-0.5898037267$

$\mathrm{H},-2.8631840593,3.4033461857,0.1829775094$

$\mathrm{H}, 0.1245579927,3.981026575,3.2160936369$

$\mathrm{H},-1.9534898702,4.7125083845,2.0805370366$

C, $1.8752254468,0.0000008926,-4.0381029631$

C, $1.2977453381,1.2173242224,-2.0349346346$

C, $1.2977465635,-1.2173232773,-2.0349347898$

C, $1.6831723587,-1.2098313596,-3.3716397477$

C, $1.6831711376,1.2098328611,-3.3716396077$

H,1.1490087572,2.160687501,-1.5234161933

H,1.1490108855,-2.1606867651,-1.5234164698

H,1.8323602863,-2.1482411547,-3.8917683504

H, 1.8323580774,2.1482428722,-3.8917680977

H,2.174637182,0.0000010871,-5.0795204953

$\mathrm{HF}=-1036.8790621 \quad \mathrm{NImag}=1$

Me2PhP H+

phme $2 \mathrm{p}+1 . \log$.acs

Brutoformula: C8H12P1(1+)

1,1

C, $-0.2197885385,0.0000000148,-0.3651093668$

P,-0.4608004704,-0.0000000031,1.4119238015

$\mathrm{H},-1.8496965471,-0.0000000054,1.6159105144$

C, $0.2033671092,1.4865872704,2.2112478634$

C, $0.2033671126,-1.4865872811,2.211247857$

C, $0.1357873689,-0.0000000076,-3.1220717434$ 
C, $-0.4394441683,-0.0000000029,-0.2160315676$ $\mathrm{H}, 1.7115813447,-0.000000032,-0.000196318$ $\mathrm{H}, 0.295071147,0.0000000128,-4.0429743609$ H,-2.0484077719,0.0000000104,-3.2242343543 $\mathrm{P},-0.8877942983,0.0000000004,1.5887216095$ H,-2.5104893208,-0.0000000113,-0.7933902069 C, $0.1442633481,-1.422574518,2.2111642934$ C, $0.1442633436,1.4225745243,2.2111642892$ H,1.2014113658,-1.3396398083,1.9444479091 $\mathrm{H},-0.2494472893,-2.356614709,1.8051166953$ $\mathrm{H}, 0.0595935042,-1.46684549,3.3001594057$ H,1.2014113605,1.339639821,1.944447899 $\mathrm{H}, 0.0595935033,1.4668454971,3.3001594017$ $\mathrm{H},-0.2494473009,2.356614713,1.8051166913$ $H F=-652.9421366$ NImag $=0$
C,-1.3381027816,0.0000000382,-1.2111909523

C, $1.078077061,0.0000000322,-0.9000014229$

C, $1.2485187619,-0.00000002,-2.2793915079$

C,-1.1523119963,-0.0000000157,-2.5907241949

H,-2.3442985403,0.0000000516,-0.8069671754 $\mathrm{H}, 1.9524861007,0.0000000353,-0.2577299518$

$\mathrm{H}, 2.2480025813,-0.0000000218,-2.6966803373$

H,-2.0135908092,-0.0000000435,-3.2474485869 $\mathrm{H}, 0.2751722158,-0.0000000075,-4.1965272349$

$\mathrm{H},-0.2457246033,-2.3718322151,1.7585113731$

H,-0.0229719598,-1.4658033154,3.2792019133

H, 1.2856046564,-1.525905998,2.0740550679

$\mathrm{H},-0.2457246157,2.3718322076,1.7585113944$

$\mathrm{H}, 1.2856046518,1.5259059958,2.0740550655$

H,-0.0229719555,1.4658032884,3.27920192

$\mathrm{HF}=-653.317471 \quad \mathrm{NImag}=0$ 